\author{
Universidade de São Paulo \\ Instituto de Física de São Carlos
}

William Couto Corrêa de Lima

\title{
Análogos de gravitação semi-clássica em física da matéria condensada
}



William Couto Corrêa de Lima

\section{Análogos de gravitação semi-clássica em física da matéria condensada}

Dissertação apresentada ao Instituto de Física de São Carlos da Universidade de São Paulo para obtenção do título de Mestre em Ciências.

Área de concentração: partículas e campos, relatividade geral.

Orientador: Daniel Augusto Turolla Vanzella.

São Carlos 

Folha de Aprovação 



\section{Dedicatória}

Dedico este estudo ao povo do Estado de São Paulo que, a despeito das dificuldades, com seus impostos apóia e financia, através de instituições como a Universidade de São Paulo e a Fundação de Amparo à Pesquisa do Estado de São Paulo, parte significativa da pesquisa científica brasileira. 



\section{Agradecimentos}

Agradeço aos professores e funcionários do Instituto de Física de São Carlos, onde realizei meus estudos de graduação e de mestrado. Agradeço à Fundação de Amparo à Pesquisa do Estado de São Paulo (FAPESP) pelo apoio financeiro a este estudo. Agradeço aos amigos G. C. P. Innocentini e G. M. Nakamura pelos agradáveis momentos do cafezinho e as interessantes discussões e opiniões. Agradeço aos amigos que tenho desde os idos e vividos tempos do colégio e que, a despeito da distância física, sempre estão próximos. A eles o meu muito obrigado pelos momentos bons e a amizade sincera. Agradeço aos amigos E. W. Merheb e J. H. Pettená do Carmo por todos os bons anos que moramos juntos. Agradeço aos meus pais pelo inestimável apoio, em todos os sentidos, de toda uma vida. Por fim, agradeço enormemente ao Prof. Dr. D. A. T. Vanzella, o Patrão, por todos os ensinamentos, dicas, discussões e pontos de vista que certamente reverberarão por toda minha carreira científica. Os cafés na Química e os almoços no Bandejão certamente ficarão na memória. 

"Conhecer não é suficiente, é preciso aplicar. Vontade não é suficiente, temos de fazer".

Johann Wolfgang von Goethe (1749 - 1832)

"Em todas as coisas da Natureza há algo de maravilhoso".

Aristóteles (384 a.C. - 322 a.C.)

"A ciência são fatos; assim como casas são feitas de madeira e pedras, a ciência é feita de fatos; mas um amontoado de pedras não é uma casa e uma coleção de fatos não necessariamente é ciência".

Jules Henri Poincaré (1854 - 1912)

"No it can't! An argument is a connected series of statement intended to establish a proposition".

Monty Python no quadro The Argument Clinic 



\section{Resumo}

\section{LIMA, W.C.C. Análogos de gravitação semi-clássica em física da matéria con-}

densada. 2008. 126 p. Dissertação (Mestrado) - Instituto de Física de São Carlos, Universidade de São Paulo, São Carlos, 2008.

A presente dissertação tem como objeto de estudo sistemas da física da matéria condensada que sejam capazes de simular sistemas gravitacionais, tais como buracos negros e universos em expansão, onde processos quânticos tomam parte. Neste estudo nos debruçamos principalmente sobre o modelo do fluido e condensados de Bose-Einstein. No modelo do fluido exploramos a geometria efetiva que surge e os problemas de back-reaction e dos modos trans-planckianos de campos quânticos. No modelo baseado em condensados exploramos sua faceta cosmológica e a possibilidade de campos maciços. Além desses dois modelos de grande relevância na literatura, ainda expomos os análogos em cordas elásticas e os baseados em ondas na superfícies de fluidos e uma análise geral baseada no formalismo lagrangeano para campos.

Palavras-chave: acústica, relatividade geral, buracos mudos, buracos negros, cosmologia, mecânica quântica, teoria quântica de campos em espaços curvos, gravitação semi-clássica, efeito Hawking. 



\begin{abstract}
LIMA, W.C.C. Analogue models of semi-classical gravity in condensate matter physics. 2008. 126 p. Thesis (Master) - Instituto de Física de São Carlos, Universidade de São Paulo, São Carlos, 2008.

This dissertation has as object of study systems of condensate-matter physics which can simulate gravitational systems like black holes and expanding universes where quantum processes take place. In this study we lay attention mainly on the fluid model and on Bose-Einstein-condensate-based models. In the fluid model we explore the features of the emergent geometry and other problems like the back-reaction and the trans-planckian modes of quantum fields. In the condensate-based models we explore their cosmological aspects and the possibility for massive fields. Moreover, we shall present two other models, the elastic string and the surface-wave-based models in fluids, and a very general analysis based on the Lagrangean formalism for fields.
\end{abstract}

Keywords: acustic, general relativity, dumb holes, black holes, cosmology, quantum mechanics, quantum field theory in curved spaces, semiclassical gravitation, Hawking effect. 



\section{Sumário}

1 Introdução

2 Rudimentos de teoria de campos

2.1 Um pouco sobre TQC

2.1.1 Equação de Klein-Gordon

2.1.2 Quantizacão canônica .

2.1.3 Partículas em TQd .

2.1.4 Energia de ponto zero

2.1.5 Efeito Casimin

2.1.6 Efeito Unruh

2.2

2.2.1 Quantizacão canônica em espaços-tempos curvos . . . . . . . . . . . 35

2.2 .2 Partículas em TQCEd . . . . . . . . . . . . . . . . 36

2.2 .3 Efeito Hawking . . . . . . . . . . . . . . . . . . . . . . . . . . . 40

$3 \quad$ Analogia em fluidos 51

3.1 Som em fluidos ideais

3.1.1 Equacões para o fluido

3.1.2 Equacão para o som

3.1.3 Analogia com a RG

3.1.4 Espaco-tempo newtoniano versus espaco-tempo efetivo

3.2 Espacos-tempos acústicos: algumas propriedades

3.2.1 Signatura da métrica acústica

3.2.2 4-velocidade e 4-aceleração 40

6 
$3.2 .3 \quad$ Estabilidade causal . . . . . . . . . . . . . . . . . . . 61

3.2 .4 Geodésicas tipo "som" . . . . . . . . . . . . . . . . . . . 61

3.3 Espacos-tempos acústicos: algumas definições $\ldots \ldots$. . . . . . . . . . . . 62

3.3.1 Espaço-tempo acústico estacionário . . . . . . . . . . . . . . . . . 62

3.3.2 Espaço-tempo acústico estático . . . . . . . . . . . . . . . . . . 63

3.3 .3 Ergoregião . . . . . . . . . . . . . . . . . . . . . . 64

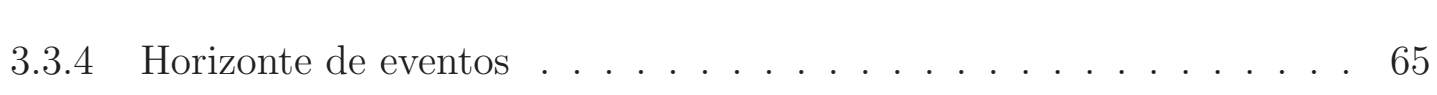

3.3.5 Gravidade superficial . . . . . . . . . . . . . . . . . 66

3.4 Adicionando viscosidade $\ldots \ldots \ldots \ldots$. . . . . . . . . . . . . 67

3.5 Alguns apontamentos experimentais . . . . . . . . . . . . . . 69

4 Aplicacões do modelo do fluido $\quad 73$

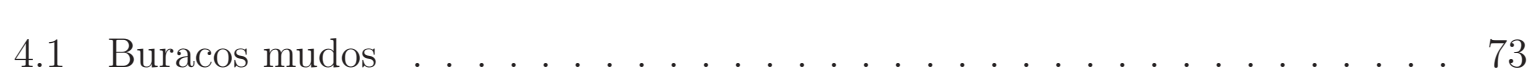

4.1 .1 Fluxos unidimensionais . . . . . . . . . . . . . . . . . . 73

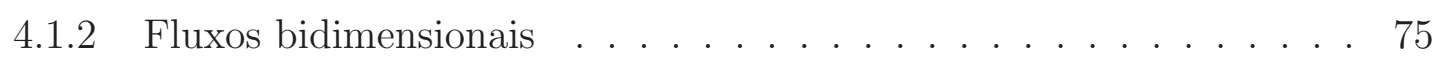

4.1 .3 Fluxos tridimensionais . . . . . . . . . . . . . . . 76

4.2 Radiação Hawking no bocal de Laval . . . . . . . . . . . . . . . . . . . . . 79

4.3 Back-reaction em análogos $\ldots \ldots$. . . . . . . . . . . . . . . . . . . . . . 81

4.4 Modos trans-planckianos em análogos $\ldots \ldots$. . . . . . . . . . . 85

5 Modelos análogos em condensados de Bose-Einstein 91

5.1 Equação de Gross-Pitaevskii $\ldots \ldots \ldots$. . . . . . . . . . . . . . . 92

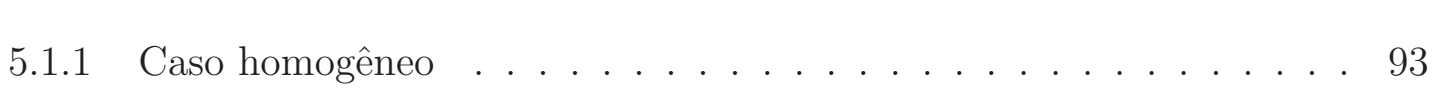

5.1 .2 Caso não-homogêned . . . . . . . . . . . . . . . . . . . . . 97

5.2 Regime hidrodinâmico $\ldots \ldots \ldots \ldots$. . . . . . . . . . . . . . . . . 98

5.3 Vórtices quantizados $\ldots \ldots$. . . . . . . . . . . . . . . . . . 100

5.4 Modelos cosmológicos em condensados $\ldots$. . . . . . . . . . . . . . 100

5.4 .1 Condensados em expansão . . . . . . . . . . . . . . . . . . 100

5.4 .2 Expansão via variação da secção de choque . . . . . . . . . . . . . . . 102

5.5 Teoria de Bogoliubov para excitacões em condensados . . . . . . . . . . . 104 
$\begin{array}{lll}6 & \text { Outros modelos } & 109\end{array}$

6.1 Ondas em superfícies de fluidos f . . . . . . . . . . . . . . . . . . . . 109

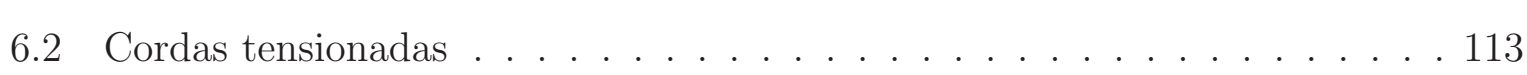

6.3 Modelo análogo via análise lagrangeana . . . . . . . . . . . . . . . 115

$\begin{array}{lll}7 & \text { Conclusão } & 119\end{array}$ 


\section{Capítulo 1}

\section{Introdução}

Em 1915 A. Einstein publicou uma das mais elegantes e profundas teorias da física para descrever a força gravitacional. A teoria da gravitação de Einstein, ou Teoria da Relatividade Geral (RG), se mostrou a teoria física adequada para descrever desde a evolução de estrelas até a do próprio Universo. Para obtê-la, Einstein utilizou o fato experimental, conhecido desde os tempos de Newton, que diz que massa inercial e massa gravitacional têm o mesmo valor. Isto o levou a conclusão de que o que entendemos por força gravitacional na verdade é a manifestação de um fato mais profundo: massa e energia modificam a geometria do uno espaço-tempo. A RG muda radicalmente a maneira com que entendemos a realidade, principalmente por tocar nas nossas noções de espaço e tempo. Isto faz dela, sem sombra de dúvidas, um marco importante na história do conhecimento humano. Entretanto, ao lançar luz sobre estas questões tão fundamentais, a RG deixa claro suas limitações em descrever a Natureza. Há dois problemas importantes nos quais são patentes tanto os sucessos como os problemas da RG: a evolução do Universo e o colapso estelar.

A Cosmologia somente firmou-se como ciência durante o último século. Tal feito foi possível graças ao arcabouço teórico e a resultados experimentais como a expansão do Universo observada por E. Hubble (1929), a descoberta da radiação cósmica de fundo por A. Penzias e R. W. Wilson (1965) e a recente descoberta da expansão acelerada do Universo (1998). A radiação cósmica de fundo, em particular, teve papel fundamental ao por um ponto final na querela que havia até os anos 60 entre o modelo do big bang 
e o modelo do universo estacionário. No contexto do modelo do big bang, a radiação cósmica de fundo é obtida de forma natural através de considerações sobre a física das interações fundamentais em um universo inicialmente quente que se torna cada vez mais frio e rarefeito. Entretanto, este fato experimental encontra dificuldades de se encaixar no modelo do universo estacionário, onde é suposto que o Cosmos sempre apresentou as estruturas que hoje observamos. Apesar do sucesso em explicar a radiação cósmica de fundo e a nucleossíntese primordial, o modelo do big bang não é capaz de descrever o que se passa em instantes arbitrariamente próximos à "grande explosão". Nesta região, observáveis como o escalar de curvatura, a temperatura e a densidade de matéria divergem.

No contexto astrofísico, o mesmo tipo de problema pode aparecer. Até o presente momento, sabemos que as estrelas são corpos celestes que têm um ciclo de vida e que ao morrerem podem dar origem a pelo menos três tipos de objetos: uma anã branca, uma estrela de nêutrons ou um buraco negro. Um dos fatores que decidem qual será o destino da estrela é a massa que resta depois do processo de ejeção de matéria durante a explosão supernova. Uma anã branca é formada por uma estrela com até 1,4 massas solares, cuja pressão gravitacional exercida é a máxima que o princípio de Pauli aplicado a elétrons consegue suster. Este limite superior para a massa de anãs brancas é conhecido por limite Chandrasekhar. Estrelas com massas superiores a este limite podem dar origem, após o colapso, às chamadas estrelas de nêutrons. Nestas estrelas o princípio de Pauli aplicado a nêutrons é que sustém a pressão gravitacional. Ainda hoje são questões em aberto a de qual é o limite para a massa da estrela a partir do qual seu colapso gera um buraco negro e a de se há ou não objetos intermediários entre estrelas de nêutrons e buracos negros. Entretanto, sabe-se que se a massa da estrela for grande o suficiente (estima-se entre 2 e 5 massas solares) seu colapso pode gerar um buraco negro. Um buraco negro é uma solução bastante exótica das equações de Einstein com duas características importantes: ele apresenta um horizonte de eventos, isto é, uma fronteira para uma região da qual nada escapa, e um "ponto" em torno do qual observáveis, como a curvatura, divergem.

Estas divergências de observáveis, ou singularidades como são conhecidas estas e outras patologias, em modelos que acreditamos ter realidade física são um fato bastante desagradável. Isto deixa claro que a RG não é capaz de descrever a realidade em certas 
escalas de tempo, tamanho e massa mas acredita-se que uma teoria quântica da gravitação o seja. Podemos estimar a ordem de grandeza destas escalas através da razoável suposição de que nesta teoria devam estar presentes a velocidade da luz $c$, que carrega seu caráter relativístico, a constante da gravitação de Newton $G$, que carrega a informação de que estamos falando em gravitação, e a constante de Planck $\hbar$, que nos diz que estamos no domínio quântico. Estas três constantes podem ser combinadas para fornecer para a teoria uma escala típica de comprimento, tempo e massa, conhecida como escala de Planck,

$$
l_{\mathrm{P}}=\sqrt{\frac{\hbar G}{c^{3}}} \sim 10^{-33} \mathrm{~cm}, \quad t_{\mathrm{P}}=\sqrt{\frac{\hbar G}{c^{5}}} \sim 10^{-44} \mathrm{~s}, \quad m_{\mathrm{P}}=\sqrt{\frac{\hbar c}{G}} \sim 10^{-5} \mathrm{~g} .
$$

Especula-se que nestas escalas a idéia de um espaço-tempo contínuo seja absolutamente desprovida de sentido. Infelizmente, como é bem sabido pela comunidade, tal formulação da gravitação ainda nos escapa. Vejamos algumas das dificuldades.

O espaço-tempo da RG é uma variedade diferenciável $M$ dotada da métrica lorentziand $g_{a b}$, o espaço métrico $\left(M, g_{a b}\right)$, cuja dinâmica é ditada pelas equações de Einstein. A métrica $g_{a b}$ na RG é o objeto matemático que codifica ao mesmo tempo a estrutura causal do espaço-tempo e o campo gravitacional da distribuição de matéria em questão. Seja $\hat{\psi}$ um campo quântico definido no espaço-tempo de Minkowski; $\left(\mathbb{R}^{4}, \eta_{a b}\right)$. Sabemos que o campo satisfaz

$$
\left[\hat{\psi}(x), \hat{\psi}\left(x^{\prime}\right)\right]=0
$$

desde que os pontos $x$ e $x^{\prime}$ sejam separados por um intervalo tipo espaço. Esta propriedade garante que medidas do estado do campo, uma feita em $x$ e outra em $x^{\prime}$, se tomadas no mesmo instante, não podem influenciar uma a outra. Em outras palavras, garante a causalidade. Agora considere que a métrica do espaço-tempo é um campo quântico. Ingenuamente, podemos propor que

$$
\left[\hat{g}_{a b}(x), \hat{g}_{c d}\left(x^{\prime}\right)\right]=0,
$$

novamente quando $x$ e $x^{\prime}$ forem separados por um intervalo tipo espaço. Entretanto, não podemos dizer que a separação entre estes pontos é tipo espaço até que tenhamos obtido

\footnotetext{
${ }^{1}$ Deste ponto em diante, a todo momento que usamos o termo métrica, está implícito que estamos falando de um tensor métrico lorentziano.
} 
qual é a métrica. Por outro lado, a relação acima deve valer independentemente do estado do campo gravitacional. Logo, o conceito de causalidade não é bem definido.

Uma possibilidade de resolver o problema acima é escrever a métrica da seguinte forma:

$$
\hat{g}_{a b}=\eta_{a b}+\hat{\gamma}_{a b}
$$

e entender a RG como uma teoria de campos para um campo auto-interagente $\hat{\gamma}_{a b}$ sobre um espaço-tempo cuja estrutura causal é ditada por $\eta_{a b}$. Há problemas que surgem deste tipo de abordagem. Um que podemos mencionar, de ordem conceitual, decorre do próprio espírito da RG. É a métrica $g_{a b}$, e não $\eta_{a b}$, que é responsável pela estrutura causal do espaço-tempo. Nesta abordagem, entretanto, caso seja possível calcular algum observável com significado, o resultado obedece a estrutura causal da métrica de Minkowski.

Quantizar o campo da gravitação torna-se um problema extremamente intrincado por dois motivos intimamente ligados. Situações em que podemos experimentar campos gravitacionais suficientemente intensos para que variações na escala de Planck sejam relevantes ainda nos são inacessíveis, seja em aceleradores de partículas ou em contextos astrofísicos. Esta lacuna de dados experimentais torna muito difícil para os físicos teóricos a arte de modelar a Natureza sobre suas escrivaninhas. Sem ter por onde se guiar, imaginar o que uma teoria quântica sobre o espaço e o tempo pode significar torna-se dos exercícios mais árduos.

A despeito dos problemas hercúleos mencionados, nos cabe não nos deixar abater. É bem conhecido que antes mesmo da Eletrodinâmica Quântica, muitos resultados da interação radiação-matéria já tinham sido obtidos através da abordagem semi-clássica. Do ponto de vista desta aproximação, supõe-se que a matéria obedece as leis da Mecânica Quântica enquanto que o campo eletromagnético é absolutamente clássico. Então, por que não adotar o mesmo procedimento com o campo gravitacional? Este tipo de idéia foi levada a cabo nos anos 60 e deu origem ao que hoje se conhece como Teoria Quântica de Campos em Espaços Curvos (TQCEC).

Talvez seja a produção de pares o efeito mais emblemático da Teoria Quântica de Campos no espaço-tempo de Minkowski (TQC). Na presença de um campo elétrico intenso é possível criarmos pares elétron-pósitron a partir do vácuo (efeito Schwinger [1]). Assim, é bastante razoável nos perguntarmos se na presença de um campo gravitacional intenso 
é também possível criarmos pares. Este efeito seria relevante no Universo primitivo? Este tipo de pergunta levou L. Parker, em meados da década de 60, a fazer a primeira extensão da TQC para espaços-tempos curvos [2]. Para obter seus resultados, Parker supôs que a gravitação é descrita por um campo clássico que obedece a RG enquanto que a matéria é descrita por campos quânticos que satisfazem a generalização covariante das equações de campo livre relativísticas. Parker obteve uma estimativa para a taxa de produção de pares no Universo atual. Ele estimou a produção de méson $\pi$ em menos de um por segundo no Universo observável $\left(\sim 10^{81} \mathrm{~cm}^{3}\right)$. Para prótons e elétrons, respectivamente, estimou em $10^{-64} \mathrm{~g} \mathrm{~cm}^{-3} \mathrm{~s}^{-1}$ e $10^{-69} \mathrm{~g} \mathrm{~cm}^{-3} \mathrm{~s}^{-1}$. A maior das taxas corresponde a menos de uma partícula por litro em $10^{30}$ anos. Um aspecto deste mecanismo é que através dele se produz iguais quantidades de matéria e anti-matéria.

Na mesma época surgiam vários resultados interessantes sobre buracos negros. Depois da publicação de sua versão final e de seu sucesso em explicar a precessão do periélio de Mercúrio em 1915, da verificação do desvio da luz por Sir A. S. Eddington em 1919 e de explicar os resultados de Hubble no final da década de 20, a RG ficou até os anos 50 sem receber grande atenção. A partir do final dos anos 40, graças ao desenvolvimento das antenas de rádio por conta da Segunda Guerra Mundial, apareceu um novo ramo da astronomia: a radioastronomia. Com novas técnicas, a radioastronomia permitiu aos astrônomos vislumbrar objetos que emitem radiação fora da faixa do visível. Muitos dos objetos que passaram a ser vistos eram muito mais densos do que as estrelas conhecidas. Tais observações suscitaram pesquisas sobre corpos compactos e colapsos estelares, assuntos para os quais a RG é indispensável. Este cenário motivou, durante os anos 60 e 70, um enorme desenvolvimento tanto no formalismo da RG como no entendimento de algumas de suas soluções, principalmente no que se refere a buracos negros.

Em 1969 R. Penrose descobriu uma forma clássica de extrair energia de um buraco negro em rotação. O mecanismo que Penrose concebeu é o seguinte. Imagine uma partícula que após adentrar à ergosfera de um buraco negro girante, uma região na qual o campo gravitacional é tal que os corpos que ali estão não têm opção senão seguir o movimento de rotação, se decomponha em duas outras, uma que passa a descrever uma órbita de energia negativa (e que depois é engolida pelo buraco) e outra que escapa da ergosfera. 
A partícula que escapa, por conservação, carrega para longe parte da energia de rotação do buraco. Este é o efeito Penrose. Pouco depois da descoberta de Penrose, Misner, Zel'dovich [3] e Starobinskii [4] conceberam um efeito análogo ao efeito Penrose para ondas espalhadas por buracos negros girantes. Este efeito para ondas é conhecido como superradiância. No contexto quântico, a superradiância implica na emissão espontânea de partículas pelo buraco, o que já era esperado e foi confirmado por W. G. Unruh [5]. Em 1973, J. D. Bekenstein, tendo em vista uma série de resultados obtidos para buracos negros e o aparente conflito entre eles e a segunda lei da Termodinâmica, propôs que a estes objetos estaria associada uma entropia proporcional a área do horizonte [6]. Até então, possíveis semelhanças entre a física de buracos negros e as leis da Termodinâmica eram tratadas como mero acaso. S. W. Hawking argumentou, pouco depois que, a despeito das semelhanças, não poderíamos atribuir uma entropia a buracos negros já que a temperatura deles era rigorosamente zero [7]. Entretanto, em 1974 o próprio Hawking viu-se obrigado a mudar de posição [8], 9]. Através de um cálculo mais acurado, que leva em consideração o vácuo quântico, Hawking descobriu que uma estrela colapsando e dando origem a um buraco negro é capaz de emitir partículas, mesmo que o buraco negro formado tenha momento angular zero. Este fato em si não é algo surpreendente. O que chama a atenção no resultado de Hawking é que independente da forma com que a estrela colapse, isto é, somente pelo fato de haver a formação de um horizonte de eventos, o espectro das partículas que chegam ao infinito futuro é idêntico ao de um corpo negro. O fato assombroso é a universalidade do resultado. Como um corpo negro ideal é totalmente caracterizado pela sua temperatura, um observador no infinito futuro é capaz de atribuir uma temperatura e, conseqüentemente, uma entropia ao buraco. Entropia esta que é proporcional a área do horizonte de eventos. Este efeito hoje é conhecido na literatura como efeito Hawking. A temperatura associada a radiação é dada por

$$
T_{\mathrm{H}}=\frac{\hbar \kappa}{2 \pi k_{\mathrm{B}} c}
$$

onde $k_{\mathrm{B}}$ é a constante de Boltzmann e $\kappa$ a gravidade superficial do buraco. Um buraco negro, sem carga e sem rotação, com massa da ordem da massa do Sol $\left(\sim 10^{33} \mathrm{~g}\right)$ teria uma temperatura da ordem de $10^{-6} \mathrm{~K}$, muito menor que os $2,7 \mathrm{~K}$ da radiação cósmica de fundo. 
Depois dos resultados de Parker e Hawking o que se seguiu foram investigações acerca do conceito de partícula; percebeu-se que a TQCEC prescindia do conceito de partícula para fazer predições com sentido físico. Mais tarde englobou-se a TQCEC problemas com interação entre campos e generalizou-se o conceito de renormalizaçao para espaços curvos. Atualmente a TQCEC é tão bem posta quanto a TQC é, ao menos para campos livres 2

Apesar de os resultados previstos pela TQCEC terem fundamentação matemática sólida, é imperativo que sejam verificados em laboratório. Afinal, física ainda é uma ciência essencialmente experimental. Em contextos astrofísicos e cosmológicos não temos esperanças de verificar as predições da TQCEC tão cedo. O caso da radiação Hawking é emblemático. No caso de um buraco de Schwarzschild a temperatura da radiação vai com o inverso da massa. Os corpos celestes que conhecemos que são fortes candidatos a buracos negros são muito mais maciços que o Sol. Logo, é impraticável observar a radiação Hawking produzida por estes objetos. Uma possibilidade também ainda distante do nosso alcance tecnológico, devido às escalas de energias envolvidas, é a produção de mini buracos negros em aceleradores de partículas. Como a massa deles seria muito pequena, a temperatura seria alta o suficiente para ser detectada. Ainda há uma terceira alternativa, muito mais acessível experimentalmente, de testarmos o formalismo e as predicões da TQCEC: os análogos de sistemas gravitacionais em física da matéria condensada 3 . É sobre eles que discorreremos daqui em diante.

Em linhas gerais, modelos análogos consistem em um meio material cujas quantidades que o descrevem são inomogêneas e dependentes do tempo. Neste meio está definido algum tipo de campo físico cuja dinâmica é influenciada pelas inomogeneidades. Esta influência é tal que o campo percebe as inomogeneidades como a métrica de um espaço-tempo curvo efetivo. O primeiro sistema de matéria condensada com a finalidade de simular efeitos de gravitação semi-clássica foi proposto por W. G. Unruh em 1981 [14]. Neste trabalho, Unruh mostrou que o som propagando-se num fluido com fluxo inomogêneo comporta-se como um campo escalar sem massa em um espaço-tempo curvo. Em particular, ele obteve

\footnotetext{
${ }^{2}$ Para uma revisão detalhada do desenvolvimento da TQCEC ver [10.

${ }^{3}$ Ao longo do texto, freqüentemente vamos nos referir a estes sistemas como modelos análogos ou simplesmente análogos. Para uma revisão bastante ampla sobre o tema e seu desenvolvimento ver [11] Para outros trabalhos de revisão ver 12 e [13]
} 
que a geometria efetiva gerada por um fluxo convergente que em algum momento tornase supersônico é análoga a de um espaço-tempo de Schwarzschild. Na literatura esta geometria efetiva é conhecida como buraco negro acústico ou buraco mudo. Seguindo os argumentos de Hawking, Unruh concluiu que buracos mudos devem irradiar fônons seguindo um espectro térmico caracterizado pela seguinte temperatura:

$$
T=\frac{\hbar}{2 \pi k_{\mathrm{B}}} \frac{\partial v_{r}}{\partial r}
$$

onde $c$ é a velocidade do som no meio e $v_{r}$ a velocidade do fluido 4 Este resultado, entretanto, não chamou a atenção da comunidade de pronto. Foi somente a partir dos anos 90 que modelos análogos passaram a ser um tópico de constante pesquisa. Durante este período o resultado de Unruh foi derivado independentemente por M. Visser [15] e deu-se atenção principalmente ao problema da física na escala de Planck em análogos [16], [17], [18], [19], [20], [21], às formulações precisas das noções de gravidade superficial, horizonte de eventos e ergoregião [22], [23], às analogias em superfluidos e sistemas de estado sólido 24] e às discussões sobre as implicações da entropia de Bekenstein-Hawking em análogos [25]. A partir do ano 2000 o interesse nestes sistemas cresceu enormemente, chegando a ser assunto de um workshop realizado no Centro Brasileiro de Pesquisas Físicas, no Rio de Janeiro, em 2002. Muitos dos trabalhos a partir de 2000 devem-se ao modelo análogo encontrado em condensados de Bose-Einstein naquele ano [26], 27]. Condensados de Bose-Einstein revelaram-se bastante promissores para simular efeitos de TQCEC. Neles é possível construir geometrias efetivas que simulam universos em expansão [28], [29] e campos maciços [30]. Acredita-se também que sejam eles o sistema que num futuro próximo nos permitirá observar algumas predições de TQCEC em laboratório. Outros assuntos de interesse neste período foram analogias em sistemas eletromagnéticos [31] e em dielétricos quânticos ("luz lenta") [32] e o problema do back-reaction em análogos [33], [34], 35].

Apesar de pioneiro no propósito de simular efeitos de TQCEC em matéria condensada, o modelo do fluido não foi o primeiro a se utilizar de uma métrica efetiva. Em um

\footnotetext{
${ }^{4}$ Para derivar este resultado Unruh assumiu que a velocidade do som é constante no meio e que o fluxo é esfericamente simétrico. Mais adiante mostraremos que o fato de o espectro de fônons ser planckiano independe destas hipóteses.
} 
artigo publicado em 1923, W. Gordon (o mesmo da equação de Klein-Gordon) propôs uma métrica efetiva para descrever o comportamento da luz em um meio dielétrico em movimento. Talvez seja este o primeiro trabalho a lançar mão do conceito.

As analogias entre sistemas gravitacionais e de matéria condensada são excelentes laboratórios teóricos e promissores laboratórios experimentais para TQCEC. Espera-se que não somente simulem efeitos da TQCEC como também nos guiem para a física que há sob o véu da escala de Planck. Assim como imagina-se que o espaço-tempo contínuo da RG perca sentido no regime trans-planckiano, sabemos que a descrição de um sistema de matéria condensada via um campo efetivo falha na escala atômica. Entretanto, diferentemente de sistema gravitacionais, conhecemos muito bem a física da qual deriva-se as teorias efetivas que descrevem sistemas de matéria condensada. É justamente por esta característica que modelos análogos têm sido motivo de intensa pesquisa nos últimos anos.

Procuramos fazer esta dissertação o mais logicamente auto-contida possível. Na elaboração assumimos que o leitor tenha conhecimentos de RG e Hidrodinâmica. Para tópicos de RG recomendamos a leitura dos primeiros capítulos de [36]. A referência [37] é um boa introdução à Hidrodinâmica; para tópicos mais avançados ver [38]. No que segue, temos o seguinte. No capítulo 2 vamos expor alguns tópicos de TQC e TQCEC pertinentes e a partir deles obter o resultado de Hawking. No capítulo 3 vamos construir o modelo do fluido, explorar a geometria emergente e definir os conceitos de gravidade superficial, horizonte de eventos e ergoregião no contexto de análogos. No capítulo 4 vamos utilizar um fluido escoando em um bocal de Laval para abordar os problemas dos modos trans-planckianos e do back-reaction. No capítulo 5 vamos exibir os análogos em condensados de Bose-Einstein partindo do hamiltoniano de muitos corpos de um sistema bosônico; faremos um contato com a teoria de Bogoliubov para excitações em condensados e mostraremos os análogos cosmológicos. No capítulo 6 exibiremos dois outros sistemas análogos a sistemas gravitacionais: o modelo da corda elástica e o baseado em ondas na superfície de fluidos. Além disso, discorreremos sobre uma análise bastante geral para análogos baseada no formalismos lagrangeano para campos. No capítulo 7 concluiremos fazendo observações pertinentes, procurando lançar questões ainda em aberto.

Ao longo desta dissertação usaremos o sistema de unidades no qual $G=c=\hbar=1$, 
entretanto, no capítulo 5, explicitaremos a constante de Planck. Utilizaremos a signatura $(-+++)$ para o tensor métrico. A partir do capítulo 3 utilizaremos a letra $c$ para denotar a velocidade do som em relação ao meio. Deste capítulo em diante estaremos utilizando ao mesmo tempo quantidades definidas no espaço-tempo euclidiano de fundo e no espaço-tempo acústico arbitrário. Denotaremos o produto escalar entre vetores do espaço euclidiano através do símbolo · e o produto escalar entre vetores definidos no espaço-tempo acústico através da contração de suas componentes com a métrica acústica. Para escalares, vetores e tensores definidos sobre o espaço-tempo usaremos a notação de índices abstrata como definida na seção 2.4 do capítulo 2 de [36]. Os vetores definidos sobre o espaço euclidiano tridimensional estarão em negrito; por exemplo, o vetor de onda denotaremos por $\mathbf{k}$. Um vetor $\mathbf{v}$ terá seu módulo denotado por v e componentes denotadas por $\mathrm{v}_{x}, \mathrm{v}_{y}$ e $\mathrm{v}_{z}$ ou por $\mathrm{v}_{i}$. Adotaremos a convenção de soma da RG. Eventualmente, vamos escrever as componentes de tensores de posto $(0,2)$ ou $(2,0)$ na forma de matriz. Como é usual em RG, utilizaremos o mesmo símbolo para denotar as componentes e a matriz. A unidade imaginária $\sqrt{-1}$ será denotada por i. 


\section{Capítulo 2}

\section{Rudimentos de teoria de campos}

Neste capítulo vamos expor alguns tópicos de TQC e TQCEC que serão pertinentes mais adiante. Iremos procurar fazê-lo da forma mais simples possível, frisando o caráter operacional da teoria no espírito de [39] e [40] (para uma abordagem mais rigorosa, ver [41]; para uma abordagem didática, ver [42]). Por simplicidade, vamos utilizar um campo escalar maciço hermitiano para fixar as idéias. Além da simplicidade, temos interesse no campo escalar pois é ele que descreve as perturbações sobre os sistemas de matéria condensada que iremos tratar.

Na seção 2.1 utilizaremos o formalismo canônico para quantizar o campo escalar maciço. Estaremos preocupados em discutir um pouco sobre o estado de vácuo em TQC e o conceito de partícula em espaços planos. Neste sentido, aplicaremos o formalismo desenvolvido para reproduzir o efeito Casimir e o efeito Unruh. O resultado de Casimir servirá para ilustrarmos que o vácuo quântico tem sentido físico e que ao perturbá-lo podemos gerar efeitos interessantes. Utilizaremos o efeito Unruh para mostrar que em TQC o conceito global de partículas está intrinsecamente atrelado às simetrias do espaço-tempo de Minkowski.

Na seção 2.2, seguindo os mesmos passos desenvolvidos para espaços planos, quantizaremos o campo escalar maciço definido sobre uma geometria curva. Aqui desejamos discutir um pouco sobre o conceito de partículas quando efeitos gravitacionais tornam-se importantes. Falaremos um pouco sobre a criação de pares por efeitos gravitacionais e descreveremos as tecnicalidades por trás da derivação do efeito Hawking. 


\subsection{Um pouco sobre TQC}

Em TQC, assim como em toda a física em espaço plano, sabemos que qualquer lei física deve ser invariante sob transformações próprias e ortócronas de Poincaré. Isto decorre do fato de o espaço-tempo de Minkowski apresentar as simetrias do grupo de Poincaré e de que o subgrupo próprio e ortócrono ser a parte conexa que contém a identidade. Este é o princípio da covariância especial: as leis físicas devem ter as mesmas simetrias que o espaço-tempo sobre o qual estão definidas. O princípio da covariância especial, aplicado ao espaço-tempo de Minkowski, implica no fato experimental de que qualquer família de observadores inerciais deve observar a mesma física. Utilizando uma das famílias de observadores privilegiadas pelo grupo de simetria, podemos construir um sistema de coordenadas global sobre o espaço-tempo. Para isto, basta usarmos o tempo próprio e as distâncias próprias que estes observadores medem como, respectivamente, a coordenada temporal $t$ e as coordenadas espaciais $\mathbf{x}$, com uma escolha arbitrária da origem de ambas. Este é o sistema de coordenadas que usaremos para levar a cabo a quantização do campo escalar.

\subsubsection{Equação de Klein-Gordon}

Considere um campo escalar maciço real $\phi$ definido em todo ponto $x$ do espaço-tempo $\left(\mathbb{R}^{4}, \eta_{a b}\right)$. A ação deste campo é dada por

$$
S=-\frac{1}{2} \int\left(\eta^{a b} \partial_{a} \phi \partial_{b} \phi+m^{2} \phi^{2}\right) d^{4} x
$$

onde $m$ é a massa do quantum do campo. O momentum canonicamente conjugado a $\phi$ é definido por

$$
\pi=\frac{\delta S}{\delta\left(\partial_{t} \phi\right)}=\partial_{t} \phi
$$

Extremizando o funcional (2.1) obtemos a seguinte equação para o campo:

$$
\square \phi-m^{2} \phi=0 \text {. }
$$

Esta equação também é conhecida como equação de Klein-Gordon.

No sistema de coordenadas utilizado, um conjunto possível de soluções para (2.3) é

$$
u_{\mathbf{k}} \propto e^{\mathrm{i}(\mathbf{k} \cdot \mathbf{x}-\omega t)}, \quad \mathbf{k} \in \mathbb{R}^{3} .
$$


Substituindo as soluções do tipo (2.4) em (2.3) vem a relação entre os parâmetros $\omega$ e k dada por

$$
\omega= \pm \sqrt{\mathrm{k}^{2}+m^{2}}
$$

Segundo a relação de dispersão (2.5), para cada $\mathbf{k}$ temos associado dois valores para w. Em Mecânica Quântica, a freqüência da onda que descreve a partícula, a menos de um fator constante, é a energia da própria partícula. Logo, como desejamos descrever partículas reais, temos que impor que $\omega$ é positivo. Os modos (2.4) com $\omega>0$ são ditos de freqüência positiva com respeito a coordenada temporal $t$. Eles são auto-funções do campo de Killing tipo tempo $\left(\partial_{t}\right)^{a}$ do espaço-tempo de Minkowski e satisfazem

$$
\partial_{t} u_{\mathbf{k}}=-\mathrm{i} \omega u_{\mathbf{k}}, \quad \operatorname{com} \omega>0 .
$$

Campos de Killing são aqueles campos vetoriais relacionados às simetrias do espaçotempo. Para um espaço-tempo arbitrário, não temos como garantir a existência de um campo de Killing tipo tempo. Isto implica que nem sempre é possível encontrar um conjunto de soluções com freqüência bem definida.

Uma vez tendo em mãos um conjunto de soluções para a equação de campo, devemos adequá-las às condições de contorno do problema. Por simplicidade, vamos impor condições de contorno periódicas nas três direções espaciais. Assim, os possíveis valores de $\mathbf{k}$ são

$$
\mathbf{k}=\frac{2 \pi}{L}\left(n_{1}, n_{2}, n_{3}\right), \quad \operatorname{com} n_{i}=0, \pm 1, \pm 2, \ldots
$$

onde $L$ é o tamanho da caixa nas três direções espaciais.

Ainda falta fixarmos a constante de proporcionalidade em (2.4). Por conta da interpretação probabilística, as ondas que descrevem as partículas em mecânica quântica devem ser normalizadas. A equação (2.3) define o seguinte produto interno para o espaço formado pelo seu conjunto de soluções:

$$
\left(\phi_{1}, \phi_{2}\right)=-\mathrm{i} \int_{t}\left(\phi_{1} \partial_{t} \phi_{2}^{*}-\partial_{t} \phi_{1} \phi_{2}^{*}\right) d^{3} x=-\mathrm{i} \int_{t} \phi_{1} \overleftrightarrow{\partial_{t}} \phi_{2}^{*} d^{3} x, \underline{1}
$$

\footnotetext{
${ }^{1}$ Note que, pela definição dada, o produto interno de Klein-Gordon $\left(\phi_{1}, \phi_{2}\right)$ é linear no primeiro argumento e anti-linear no segundo. Já o produto interno do espaço de Fock $\left\langle\psi_{1} \mid \psi_{2}\right\rangle$ é anti-linear no primeiro argumento e linear no segundo. Esta diferença é uma mera convenção e foi adotada aqui para sermos coerentes com aquela adotada em [40].
} 
onde o sub-índice $t$ no sinal de integração indica que a integral é calculada sobre alguma superfície de simultaneidade da família de observadores, e o resultado independe da superfície escolhida. O produto interno (2.8) é freqüentemente denominado de produto interno de Klein-Gordon. Através dele, definimos a constante de proporcionalidade em (2.4) impondo que

$$
\left(u_{\mathbf{k}}, u_{\mathbf{k}^{\prime}}\right)=\delta_{\mathbf{k}, \mathbf{k}^{\prime}}
$$

De (2.9) decorre que a constante de proporcionalidade é tal que os modos têm a seguinte forma

$$
u_{\mathbf{k}}=\frac{1}{\sqrt{2 L^{3} \omega}} e^{\mathrm{i}(\mathbf{k} \cdot \mathbf{x}-\omega t)} .
$$

Utilizando a base de soluções (2.10) e seu complexo conjugado podemos expandir qualquer solução de (2.3).

\subsubsection{Quantização canônica}

A quantização canônica nos dá a seguinte prescrição: interpretar $\phi$ e $\pi$ como operadores e impor as seguintes relações de comutação:

$$
\left[\hat{\phi}(t, \mathbf{x}), \hat{\phi}\left(t, \mathbf{x}^{\prime}\right)\right]=0 \quad\left[\hat{\pi}(t, \mathbf{x}), \hat{\pi}\left(t, \mathbf{x}^{\prime}\right)\right]=0 \quad\left[\hat{\phi}(t, \mathbf{x}), \hat{\pi}\left(t, \mathbf{x}^{\prime}\right)\right]=\mathrm{i} \delta\left(\mathbf{x}-\mathbf{x}^{\prime}\right)
$$

Como as soluções da forma (2.10) formam um espaço vetorial completo munido do produto interno (2.8), podemos expandir os operadores $\hat{\phi}$ e $\hat{\pi}$ em termos delas da seguinte maneira:

$$
\begin{gathered}
\hat{\phi}(t, \mathbf{x})=\frac{1}{\sqrt{2 L^{3} \omega}} \sum_{\mathbf{k}}\left[\hat{a}_{\mathbf{k}} e^{i(\mathbf{k} \cdot \mathbf{x}-\omega t)}+\hat{a}_{\mathbf{k}}^{\dagger} e^{-\mathrm{i}(\mathbf{k} \cdot \mathbf{x}-\omega t)}\right] \\
\hat{\pi}(t, \mathbf{x})=-\mathrm{i} \sqrt{\frac{\omega}{2 L^{3}}} \sum_{\mathbf{k}}\left[\hat{a}_{\mathbf{k}} e^{\mathrm{i}(\mathbf{k} \cdot \mathbf{x}-\omega t)}-\hat{a}_{\mathbf{k}}^{\dagger} e^{-\mathrm{i}(\mathbf{k} \cdot \mathbf{x}-\omega t)}\right] .
\end{gathered}
$$

Substituindo (2.12) em (2.11) vêm as relações de comutação para os operadores $\hat{a}_{\mathbf{k}}$ e $\hat{a}_{\mathbf{k}}^{\dagger}$

$$
\left[\hat{a}_{\mathbf{k}}, \hat{a}_{\mathbf{k}^{\prime}}\right]=0 \quad\left[\hat{a}_{\mathbf{k}}^{\dagger}, \hat{a}_{\mathbf{k}^{\prime}}^{\dagger}\right]=0 \quad\left[\hat{a}_{\mathbf{k}}, \hat{a}_{\mathbf{k}^{\prime}}^{\dagger}\right]=\delta_{\mathbf{k}, \mathbf{k}^{\prime}}
$$

Em TQC é conveniente, para campos livres, trabalharmos na picture de Heisenberg. Nesta picture, os operadores evoluem no tempo enquanto o estado do sistema mantém-se o mesmo. Como um campo é um objeto com infinitos graus de liberdade, isto é, é capaz 
de descrever um número qualquer de partículas, é necessário utilizarmos a representação de Fock para seu espaço de configurações. Nesta representação, os vetores do espaço de configurações do campo carregam a informação de quantas partículas ocupam um certo estado do espaço de Hilbert de uma partícula. No caso de um campo livre em espaço plano, um bom número quântico para indexar os estados no espaço de Hilbert de uma partícula é o momentum k, já que ele é conservado. Um vetor do espaço de configurações do campo, na representação de Fock, carrega a informação de quantas partículas ocupam os possíveis estados de momentum.

Sobre este espaço atuam os operadores $\hat{a}_{\mathbf{k}}$ e $\hat{a}_{\mathbf{k}}^{\dagger}$ definidos em (2.12). O vetor de nenhuma partícula $|0\rangle$, chamado o estado de vácuo da teoria na representação $\left\{u_{\mathbf{k}}\right\}$, é definido pela forma com que estes operadores atuam sobre ele, isto é, definido por

$$
\hat{a}_{\mathbf{k}}|0\rangle=0
$$

qualquer que seja k. Das relações (2.13) obtemos as seguintes regras de atuação dos operadores $\hat{a}_{\mathbf{k}}$ e $\hat{a}_{\mathbf{k}}^{\dagger}$ sobre os vetores $\left|n_{\mathbf{k}}\right\rangle$, autovetores do operador $\hat{a}_{\mathbf{k}}^{\dagger} \hat{a}_{\mathbf{k}}$ com autovalor $n_{\mathbf{k}}$ :

$$
\begin{gathered}
\hat{a}_{\mathbf{k}}\left|n_{\mathbf{k}}\right\rangle=\sqrt{n_{\mathbf{k}}}\left|(n-1)_{\mathbf{k}}\right\rangle, \\
\hat{a}_{\mathbf{k}}^{\dagger}\left|n_{\mathbf{k}}\right\rangle=\sqrt{(n+1)_{\mathbf{k}}}\left|(n+1)_{\mathbf{k}}\right\rangle,
\end{gathered}
$$

onde $n_{\mathbf{k}} \in \mathbb{N}$, qualquer que seja $\mathbf{k}$. Com isto, podemos construir um estado de ocupação qualquer do campo

$$
\left|{ }^{1} n_{\mathbf{k}_{1}},{ }^{2} n_{\mathbf{k}_{2}}, \ldots,{ }^{j} n_{\mathbf{k}_{j}}\right\rangle=\left({ }^{1} n !^{2} n ! \ldots{ }^{j} n !\right)^{-\frac{1}{2}}\left(\hat{a}_{\mathbf{k}_{1}}^{\dagger}\right)^{1} n\left(\hat{a}_{\mathbf{k}_{2}}^{\dagger}\right)^{2} n \ldots\left(\hat{a}_{\mathbf{k}_{j}}^{\dagger}\right)^{j} n|0\rangle
$$

onde os fatores ${ }^{i} n$ ! devem-se ao fato de que todos os operadores que aparecem atuando em $|0\rangle$ comutam, o que reflete a estatística de Bose para estas partículas.

\subsubsection{Partículas em TQC}

Para deixar clara a interpretação de $n_{\mathbf{k}}$ como o número de partículas ocupando o estado de momentum $\mathbf{k}$, utilizaremos o tensor energia-momentum do campo escalar. Ele é dado 
por

$$
T_{a b}=\partial_{a} \phi \partial_{b} \phi-\frac{1}{2} \eta_{a b} \eta^{c d} \partial_{c} \phi \partial_{d} \phi-\frac{1}{2} m^{2} \phi^{2} \eta_{a b}
$$

As densidades de hamiltoniana e de momentum do campo são, respectivamente, as seguintes componentes de $T_{a b}$ :

$$
\begin{gathered}
T_{t t}=\frac{1}{2}\left[\pi^{2}+(\nabla \phi)^{2}+m^{2} \phi^{2}\right], \\
T_{t i}=\pi \partial_{i} \phi .
\end{gathered}
$$

O operador hamiltoniano e as componentes do operador momentum são obtidas, respectivamente, substituindo as funções $\phi$ e $\pi$ em (2.18) pelos operadores (2.12) e integrando sobre todo volume de alguma hiperfície de simultaneidade $t=$ cte como segue:

$$
\left.\hat{H}=\int_{t} \hat{T}_{t t} d^{3} x=\sum_{\mathbf{k}} \omega\left(\hat{a}_{\mathbf{k}}^{\dagger} \hat{a}_{\mathbf{k}}+\frac{1}{2}\right) \quad \hat{\mathrm{p}}_{i}=\int_{t} \hat{T}_{t i} d^{3} x=\sum_{\mathbf{k}} \hat{a}_{\mathbf{k}}^{\dagger} \hat{a}_{\mathbf{k}} k_{i}\right]_{3}^{3}
$$

É fácil ver que $\hat{H}$ e $\hat{p}_{i}$ comutam com os seguintes operadores.

$$
\begin{gathered}
\hat{N}_{\mathbf{k}}=\hat{a}_{\mathbf{k}}^{\dagger} \hat{a}_{\mathbf{k}}, \quad \hat{N}=\sum_{\mathbf{k}} \hat{a}_{\mathbf{k}}^{\dagger} \hat{a}_{\mathbf{k}}, \\
{[\hat{N}, \hat{H}]=\left[\hat{N}, \hat{p}_{i}\right]=0 .}
\end{gathered}
$$

O significado dos operadores $\hat{N}$ e $\hat{N}_{\mathrm{k}}$ emerge quando calculamos seu valor esperado num estado de ocupação qualquer

$$
\begin{gathered}
\left\langle{ }^{1} n_{\mathbf{k}_{1}},{ }^{2} n_{\mathbf{k}_{2}}, \ldots,{ }^{j} n_{\mathbf{k}_{j}}\left|\hat{N}_{\mathbf{k}_{i}}\right|{ }^{1} n_{\mathbf{k}_{1}},{ }^{2} n_{\mathbf{k}_{2}}, \ldots,{ }^{j} n_{\mathbf{k}_{j}}\right\rangle={ }^{i} n, \\
\left\langle{ }^{1} n_{\mathbf{k}_{1}},{ }^{2} n_{\mathbf{k}_{2}}, \ldots,{ }^{j} n_{\mathbf{k}_{j}}|\hat{N}|{ }^{1} n_{\mathbf{k}_{1}},{ }^{2} n_{\mathbf{k}_{2}}, \ldots,{ }^{j} n_{\mathbf{k}_{j}}\right\rangle=\sum_{i}{ }^{i} n,
\end{gathered}
$$

e, em particular, no estado de vácuo

$$
\left\langle 0\left|\hat{N}_{\mathbf{k}_{i}}\right| 0\right\rangle=0, \quad \forall \mathbf{k}
$$

\footnotetext{
${ }^{2} \mathrm{~A}$ definição do tensor energia-momentum surge da imposição de que a ação do campo deve ser invariante sob difeomorfismos da variedade nela mesma. Esta simetria da ação dá origem, de acordo com o teorema de Noether, a correntes conservadas, que são relacionadas às componentes (e combinações de) $T_{\mu \nu}$ no sistema de coordenadas inercial. Para mais detalhes ver página 456 de [36].

${ }^{3}$ Como os operadores $\hat{\phi}$ e $\hat{\pi}$ não comutam, temos que a maneira mais natural de transformarmos $T_{t i}$ em um operador é através de $\hat{T}_{t i}=\frac{1}{2}\left(\partial_{i} \hat{\phi} \hat{\pi}+\hat{\pi} \partial_{i} \hat{\phi}\right)$. Isto implica em um termo proporcional a $\mathbf{k}$ na somatória, que é nulo.
} 
Estas relações entre $\hat{N}$ e $\hat{N}_{\mathbf{k}}$ e os números de ocupação sugerem o nomes de operador número para o modo $\mathbf{k}$ para $\hat{N}_{\mathbf{k}}$ e operador número total para $\hat{N}$. Pela (2.19), os vetores (2.16) também são auto-vetores de $\hat{H}$ e $\hat{p}_{i}$, já que estes operadores são combinações de $\hat{N}_{\mathbf{k}}$. Então, as entradas ${ }^{i} n$ podem ser interpretadas como o número de quanta de massa $m$ com energia $\omega_{i}$ e momentum $\mathbf{k}_{i}$ que estão presentes no estado indexado por $\mathbf{k}_{i}$. Isto é o que nos permite introduzir o conceito de partículas em TQC.

Como são os operadores $\hat{a}_{\mathbf{k}}$ e $\hat{a}_{\mathbf{k}}^{\dagger}$ que, quando aplicados sobre um estado, variam o número de ocupação, eles são conhecidos, respectivamente, como operadores de destruição e criação. O operador de destruição é aquele que destrói modos com freqüência positiva enquanto que o de criação é aquele que "destrói modos de freqüência negativa", ou seja, cria modos de freqüência positiva. Heuristicamente, os modos de freqüência negativa são entendidos como as anti-partículas.

Vamos refazer a construção lógica do conceito de partícula de trás para frente. Em última análise, o conceito de partícula em TQC emerge das relações de comutação (2.13) 4 4 Estas relações, por sua vez, decorrem de (2.11) e da expansão (2.12). É justamente a expansão em termos de ondas planas que nos permite interpretar as excitações dos modos normais do campo como partículas que carregam energia e momentum bem definidos. Esta expansão é possível graças a forma da equação de campo quando escrita nas coordenadas da família de observadores inerciais que escolhemos inicialmente. Conseqüentemente, o conceito de partícula está intrinsecamente atrelado ao princípio da covariância especial, ou seja, às simetrias do espaço-tempo de Minkowski.

\subsubsection{Energia de ponto zero}

Vamos calcular o valor médio do momentum e da energia do campo quando este se encontra no estado de vácuo. Para o momentum, temos

$$
\langle 0|\hat{\mathbf{p}}| 0\rangle=\mathbf{0},
$$

\footnotetext{
${ }^{4}$ Isto, pois estamos tratando de um campo que descreve partículas bosônicas. Para férmions teríamos de substituir o comutador pelo anti-comutador em (2.13).
} 
que é um resultado esperado, já que estamos olhando para o estado de nenhuma partícula. Entretanto, o valor médio do operador hamiltoniano no vácuo nos volta

$$
\langle 0|\hat{H}| 0\rangle=\sum_{\mathbf{k}} \frac{1}{2} \omega \neq 0
$$

De fato, o resultado (2.26) não é simplesmente diferente de zero, ele é infinito, posto que não há um limite superior para $\omega$. Para uma caixa suficientemente grande, temos

$$
\langle 0|\hat{H}| 0\rangle=\sum_{\mathbf{k}} \frac{1}{2} \omega=\left(\frac{L}{2 \pi}\right)^{3} \frac{1}{2} \int \omega d^{3} k=\frac{L^{3}}{(2 \pi)^{2}} \int_{0}^{\infty}\left(\mathrm{k}^{2}+m^{2}\right)^{\frac{1}{2}} k^{2} d k,
$$

e a integral é evidentemente divergente.

O fato de a integral acima divergir implica que a densidade de energia do vácuo é infinita. Isto se deve à descrição do campo como um conjunto de infinitos osciladores harmônicos. Cada um deles, em seu estado de menor energia, inevitavelmente carrega $\frac{1}{2} \omega$. Esta é uma das manifestações do princípio da incerteza de Heisenberg. Entretanto, desconsiderando gravitação, sabemos que somos somente capazes de medir diferenças entre energias. Logo, podemos contornar este problema dizendo que medimos o conteúdo de energia de um sistema a partir de sua energia de vácuo.

Se levarmos em conta fenômenos gravitacionais, temos aqui mais uma inconsistência entre RG e TQC. Do ponto de vista da RG, toda a energia do sistema deve entrar do lado direito das equações de Einstein. Se considerarmos que a energia de vácuo gravita, então a curvatura do espaço-tempo seria singular. Ingenuamente, poderíamos impor um cut-off superior para o espaço de momenta em (2.27). Poderíamos sugerir que o limite superior da integral seria da ordem de $l_{\mathrm{P}}^{-1}$ ao invés de infinito. Isto só implicaria, todavia, que a curvatura do Universo seria da ordem da escala de Planck, fato este que evidentemente não experimentamos.

\subsubsection{Efeito Casimir}

Há um argumento, devido a H. G. B. Casimir, que sugere que a energia de vácuo tem sentido físico e que seríamos capazes de detectá-la. Considere um par de chapas quadradas perfeitamente condutoras de lado $L$, separadas por uma distância $R$, com $L \gg R$, e um campo escalar não maciço em seu estado de vácuo. Suponha que sobre estas chapas o 
campo se anule. A energia $E_{L}$ do sistema sem as chapas é

$$
E_{L}=\frac{L^{3}}{(2 \pi)^{2}} \int_{0}^{\infty} k^{3} d k
$$

Quando colocamos as chapas, a condição de contorno seleciona alguns modos na região entre as elas. Suponha que o vetor normal às chapas esteja na direção do eixo $z$. A energia $E_{R}$ contida na região entre as chapas e a energia $E_{L-R}$ contida fora, respectivamente, são

$$
\begin{gathered}
E_{R}=\frac{L^{2}}{(2 \pi)^{2}} \frac{1}{2} \sum_{n=-\infty}^{n=\infty} \int_{-\infty}^{\infty} d \mathrm{k}_{x} \int_{-\infty}^{\infty} d \mathrm{k}_{y} \sqrt{\mathrm{k}_{x}^{2}+\mathrm{k}_{y}^{2}+\left(\frac{2 \pi n}{R}\right)^{2}}, \\
E_{L-R}=\frac{L^{2}(L-R)}{(2 \pi)^{2}} \int_{0}^{\infty} k^{3} d k .
\end{gathered}
$$

Assim como $E_{L}$, as energias $E_{R}$ e $E_{L-R}$ são divergentes. A diferença de energia $\Delta E$ entre o sistema com e o sistema sem as chapas é

$$
\Delta E=E_{R}+E_{L-R}-E_{L}=\frac{\pi^{2} L^{2}}{R^{3}} \int_{0}^{\infty} d x\left(\sum_{n=-\infty}^{n=\infty} \sqrt{x+n^{2}}-\int_{-\infty}^{\infty} d \xi \sqrt{x+\xi^{2}}\right) .
$$

Apesar de as parcelas serem divergentes, sua diferença não o é. De fato,

$$
\Delta E=-\frac{\pi^{2} L^{2}}{1440 R^{3}}
$$

resultado este que implica na seguinte força por unidade de área:

$$
F=-\frac{\partial}{\partial R}\left(\frac{\Delta E}{L^{2}}\right)=-\frac{\pi^{2}}{480 R^{4}}
$$

Na derivação original, Casimir utilizou o campo eletromagnético para realizar as contas e obteve um valor para a força por unidade de área que é o dobro do apresentado aqui. O fator dois se deve aos graus de liberdade de polarização do fóton.

É notável que a força obtida não dependa da constante de acoplamento entre o campo e as cargas que constituem as chapas. O efeito Casimir decorre do simples fato de que a presença das chapas distorce o vácuo do campo. Este efeito foi previsto por Casimir em 1948 e medido pela primeira vez em 1958 por M. Sparnaay nos laboratórios Philips.

\subsubsection{Efeito Unruh}

O efeito Unruh [43], 44] deixa claro que o conceito natural de partículas em TQC é totalmente dependente das simetrias do espaço-tempo de Minkowski. Exceto se estiverem 
relacionados por uma transformação de simetria do espaço-tempo, dois observadores em diferentes estados de movimentos não irão concordar sobre a presença de partículas. Logo, o conceito de partícula não tem um significado universal. Vejamos.

Considere um detector de partículas como um sistema quântico pontual onde cada nível de energia interno é indexado por sua energia E. Para simplificar, vamos supor que o detector se move em uma trajetória retilínea $z$ parametrizada pelo seu tempo próprio $\tau ; z=z(\tau)$. Considere que neste sistema há um campo escalar $\hat{\phi}$ definido em todos os pontos $x$ do espaço-tempo e que acopla com o detector através do seguinte hamiltoniano de interação:

$$
\hat{H}_{\text {int }}=c \hat{m}(\tau) \hat{\phi}[z(\tau)]
$$

Aqui, $c$ é uma constante que dá a intensidade do acoplamento e $\hat{m}$ é o operador momento de monopólo do detector. Vamos supor que no passado o campo estivesse em seu estado de vácuo $\left|0_{\mathrm{M}}\right\rangle$ e o detector em seu estado fundamental $\left|E_{0}\right\rangle$. O índice $\mathrm{M}$ em $\left|0_{\mathrm{M}}\right\rangle$ serve para lembrar que o estado de vácuo no qual o campo se encontra é aquele construído utilizandose uma família de observadores inerciais no espaço-tempo de Minkowski. Sejam $|\psi\rangle$ e $|E\rangle$, respectivamente, o estado do campo e do detector, assintoticamente, após a interação. Os estados inicial $|i\rangle$ e final $|\mathrm{f}\rangle$ do sistema são

$$
\begin{gathered}
|\mathrm{i}\rangle=\left|E_{0}\right\rangle \otimes\left|0_{\mathrm{M}}\right\rangle, \\
|\mathrm{f}\rangle=|E\rangle \otimes|\psi\rangle .
\end{gathered}
$$

Suponha que a constante $c$, em alguma escala do problema, seja pequena. Neste caso podemos usar a teoria de perturbações dependente do tempo para calcular a taxa de excitação do detector. Em primeira ordem em $c$ temos que a amplitude de transição é dada por

$$
c_{\mathrm{if}}(\tau)=-\mathrm{i} \int_{-\infty}^{\tau} d \tau^{\prime}\left\langle\mathrm{f}\left|\hat{H}_{\mathrm{int}}\right| \mathrm{i}\right\rangle .
$$

O elemento de matriz do hamiltoniano de interação é:

$$
\begin{gathered}
\left\langle\mathrm{f}\left|\hat{H}_{\text {int }}\right| \mathrm{i}\right\rangle=c\left\langle E|\hat{m}(\tau)| E_{0}\right\rangle\left\langle\psi|\hat{\phi}[z(\tau)]| 0_{\mathrm{M}}\right\rangle= \\
=c\left\langle E|\hat{m}(0)| E_{0}\right\rangle e^{-\mathrm{i}\left(E_{0}-E\right) \tau} \int \frac{d^{3} \mathrm{k}}{\left(16 \pi^{3} \omega\right)^{\frac{1}{2}}}\left\langle\psi\left|\hat{a}_{\mathbf{k}}^{\dagger}\right| 0_{\mathrm{M}}\right\rangle e^{-\mathrm{i}[\mathbf{k} \cdot \mathbf{z}(\tau)-\omega t]}
\end{gathered}
$$


Em primeira ordem, a transição só é possível se $|\psi\rangle=\left|1_{\mathbf{k}}\right\rangle$. Assim, assintoticamente no futuro, temos que $c_{\text {if }}$ é

$$
c_{\mathrm{if}}(\tau \rightarrow+\infty)=-\mathrm{i} c\left\langle E|\hat{m}(0)| E_{0}\right\rangle \int \frac{d^{3} \mathrm{k}}{\left(16 \pi^{3} \omega\right)^{\frac{1}{2}}} \int_{-\infty}^{\infty} d \tau e^{-\mathrm{i}\left(E_{0}-E\right) \tau} e^{-\mathrm{i}[\mathbf{k} \cdot \mathbf{z}(\tau)-\omega t(\tau)]} .
$$

Consideremos agora que o movimento do detector é retilíneo e uniforme. Neste caso, a equação horária é

$$
z(\tau)=z_{0}+v t(\tau)=z_{0}+\frac{v}{\sqrt{1-v^{2}}} \tau,
$$

o que implica na seguinte amplitude de transição:

$$
\begin{aligned}
c_{\mathrm{if}}(\tau & \rightarrow+\infty)=-\mathrm{i} c\left\langle E|\hat{m}(0)| E_{0}\right\rangle \int \frac{d^{3} \mathrm{k}}{\left(16 \pi^{3} \omega\right)^{\frac{1}{2}}} \int_{-\infty}^{\infty} d \tau e^{-\mathrm{i}\left[E_{0}-E+\left(k_{z} v-\omega\right)\left(1-v^{2}\right)^{-\frac{1}{2}}\right] \tau}= \\
& =-\mathrm{i} c\left\langle E|\hat{m}(0)| E_{0}\right\rangle \int \frac{d^{3} k}{\left(16 \pi^{3} \omega\right)^{\frac{1}{2}}} 2 \pi \delta\left[E-E_{0}+\left(\omega-k_{z} v\right)\left(1-v^{2}\right)^{-\frac{1}{2}}\right]=0 .
\end{aligned}
$$

De fato, pois $E>E_{0}$ e $k_{z} v<\omega$, já que $k=\sqrt{\omega^{2}-m^{2}}<\omega$ e $v<1$, o que implica que o argumento da delta é sempre positivo, independentemente de $E$ e $\mathbf{k}$. Logo, um detector cuja trajetória é inercial não é excitado quando o campo está em seu estado de vácuo.

Depois do movimento retilíneo e uniforme, o estado de movimento mais simples que podemos imaginar é o uniformemente acelerado. Um detector submetido a uma aceleração própria uniforme $\alpha^{-1}$ tem a seguinte linha de mundo:

$$
z=\left(t^{2}+\alpha^{2}\right)^{\frac{1}{2}}
$$

O tempo próprio do detector neste estado de movimento está relacionado com o tempo $t$ do laboratório por

$$
t=\alpha \sinh (\tau / \alpha)
$$

A probabilidade por unidade de tempo próprio de o sistema efetuar uma transição para algum estado de campo e algum estado do detector é

$$
p=\sum_{\mathrm{f}}\left|c_{\mathrm{if}}(\tau \rightarrow+\infty)\right|^{2}=c^{2} \sum_{E}\left|\left\langle E|\hat{m}(0)| E_{0}\right\rangle\right|^{2} \int_{-\infty}^{\infty} d(\Delta \tau) e^{-\mathrm{i}\left(E-E_{0}\right) \Delta \tau}\left\langle 0_{\mathrm{M}}\left|\hat{\phi}(x) \hat{\phi}\left(x^{\prime}\right)\right| 0_{\mathrm{M}}\right\rangle
$$

onde $x$ e $x^{\prime}$ são calculados sobre a linha de mundo do detector. Para a linha de mundo de um detector acelerado uniformemente, temos que a função de dois pontos nos dá

$$
\left\langle 0_{\mathrm{M}}\left|\hat{\phi}(x) \hat{\phi}\left(x^{\prime}\right)\right| 0_{\mathrm{M}}\right\rangle=-\left[16 \pi^{2} \alpha^{2} \sinh ^{2}\left(\frac{\Delta \tau}{2 \alpha}-\frac{\mathrm{i} \varepsilon}{\alpha}\right)\right]^{-1}
$$


onde, por simplicidade, utilizamos um campo não maciço. A pequena parte imaginária i $\varepsilon$ é escolhida para que a função de dois pontos seja a função de Wightman de freqüência positiva. Para calcular a taxa de transição devemos efetuar a transformada de Fourier da função acima, o que implica no seguinte resultado:

$$
p=\frac{c^{2}}{2 \pi} \sum_{E} \frac{\left(E-E_{0}\right)\left|\left\langle E|\hat{m}(0)| E_{0}\right\rangle\right|^{2}}{e^{2 \pi \alpha\left(E-E_{0}\right)}-1} \cdot 5
$$

O fator de Planck $\left[e^{2 \pi \alpha\left(E-E_{0}\right)}-1\right]^{-1}$ que aparece indica que quando o detector atinge o equilíbrio com o campo em seu estado de vácuo, ele responde como se estivesse em repouso na presença de um banho térmico com a seguinte temperatura:

$$
T_{\mathrm{U}}=\frac{1}{2 \pi \alpha k_{\mathrm{B}}}=\text { aceleração própria } / 2 \pi k_{\mathrm{B}}
$$

Este efeito, em princípio, pode parecer paradoxal. Como é possível que enquanto observadores inerciais não detectam partículas, observadores uniformemente acelerados sejam capazes de fazê-lo? Do ponto de vista da família de observadores inerciais, quando o detector é acelerado ele absorve energia e emite um quantum do campo. As excitações do campo e do próprio detector implicam em uma força resistiva contra a força que acelera o sistema. Assim o agente externo tem que realizar trabalho para vencer a força viscosa e manter o detector acelerando. Esta é a fonte de energia que excita o sistema. Já do ponto de vista de um observador no referencial uniformemente acelerado, o detector está parado e imerso em um banho térmico de partículas daquele campo. A incompatibilidade entre os pontos de vistas existe, pois os observadores não estão relacionados por uma transformação de simetria do espaço-tempo. De outra forma, se considerarmos que o princípio da covariância especial é o guia para leis físicas, não há motivo para que um observador

\footnotetext{
${ }^{5}$ A presença do fator de Planck na expressão para a taxa de transição entre os estados do detector é uma mera coincidência. Ele não aparecerá, por exemplo, se o campo tiver massa. Logo, o resultado obtido não implica que, na presença de qualquer outro campo, detectores com aceleração constante perceberão a presença de um banho térmico de partículas. Isto de forma alguma invalida a conclusão de Unruh. O que ocorre é que estamos olhando para a quantidade errada. De fato, para concluir que o banho de partículas é térmico deveríamos analisar a razão entre a taxa de absorção e a taxa de emissão de partículas pelo detector. Esta quantidade, também conhecida por relação de balanceamento detalhado, quando igual ao fator de Boltzmann é a "assinatura" do banho térmico. Para nosso propósito, entretanto, a conclusão apresentada é suficiente. Para mais detalhes ver 44.
} 
que seja não inercial veja a mesma física que um observador inercial. É desagradável o fato de que o conceito de partícula seja afetado por esta diferença. Isto deixa claro que esta idéia, em TQC, não é universal para as possíveis famílias de observadores.

\subsection{Um pouco sobre TQCEC}

Em TQCEC assume-se que a estrutura causal do espaço-tempo é dada por $g_{a b}$, cuja dinâmica é ditada pelas equações de Einstein, enquanto que a matéria é descrita por campos quânticos que obedecem a generalização covariante de suas equações em espaço plano. Da mesma forma que a RG, a TQCEC obedece o princípio da covariância geral. Segundo este princípio, qualquer lei física tem a mesma forma em absolutamente qualquer sistema de coordenadas. Isto é assumido pois, em espaços-tempos gerais, não há motivos para guardar nenhum tipo de simetria. Logo, não há, em geral, uma prescrição mais natural de como construir um sistema de coordenadas para recobrir o espaço-tempo. Este fato irá refletir-se na construção do vácuo do campo: se não há um sistema de coordenadas privilegiado, então não há um conjunto de modos privilegiados, o que implica que não há uma vácuo privilegiado.

\subsubsection{Quantização canônica em espaços-tempos curvos}

Considere um campo escalar real definido sobre o espaço-tempo $\left(M, g_{a b}\right)$, cuja ação é dada por

$$
S=-\frac{1}{2} \int d^{4} x \sqrt{-g}\left[g^{a b} \nabla_{a} \phi \nabla_{b} \phi+m^{2} \phi^{2}\right][6
$$

Variando a ação em termos do campo $\phi$ e impondo que a variação seja estacionária, vem a equação de campo

$$
\nabla_{a} \nabla^{a} \phi-m^{2} \phi=0
$$

\footnotetext{
${ }^{6}$ Aqui iremos considerar somente o campo escalar maciço acoplado minimamente com o campo gravitacional, isto é, sem o termo de acoplamento com o campo gravitacional em que comparece o escalar de curvatura. Para os casos que nos interessam, a saber, o colapso estelar e som se propagando em um meio não homogêneo, o termos de acoplamento conforme não comparece.
} 
Vamos supor que a geometria do espaço-tempo é tal que a equação (2.29) admita solução não trivial. A generalização do produto interno de Klein-Gordon (2.8) para espaços curvos é

$$
\left(\phi_{1}, \phi_{2}\right)=-\mathrm{i} \int_{\Sigma} \phi_{1} \overleftrightarrow{\partial_{a}} \phi_{2}^{*} n^{a} d \Sigma
$$

onde $\Sigma$ é uma hiperfície tipo espaço, $d \Sigma$ é o correspondente elemento de volume, $n^{a}$ é um campo vetorial tipo tempo normal a $\Sigma$ e unitário (ou seja, $n^{a} n_{a}=-1$ ) e orientado para o futuro.

Numa ampla classe de espaços-tempos, (2.29) possui um conjunto completo de soluções, $\left\{u_{i}(x)\right\}$, rotulados por uma coleção de índices que denotaremos por $i$, que são ortonormais segundo o produto (2.30):

$$
\left(u_{i}, u_{j}\right)=\delta_{i, j}, \quad\left(u_{i}^{*}, u_{j}^{*}\right)=-\delta_{i, j}, \quad\left(u_{i}, u_{j}^{*}\right)=0 .
$$

Adotando a prescrição da quantização canônica, vamos interpretar $\phi$ como um operador. Este operador pode ser expandido em termos do conjunto de soluções $\left\{u_{i}(x)\right\}$ de forma que

$$
\hat{\phi}(x)=\sum_{i}\left[\hat{a}_{i} u_{i}(x)+\hat{a}_{i}^{\dagger} u_{i}^{*}(x)\right]
$$

e aos operadores $\hat{a}_{i}$ e $\hat{a}_{i}^{\dagger}$ impõem-se as relações de comutação (2.13). A partir deste ponto procede-se da mesma forma que em TQC para construir-se o espaço de configurações do campo.

\subsubsection{Partículas em TQCEC}

Como mencionado antes, a interpretação de que o rótulo $n_{i}$ indica o número de partículas num certo estado $i$ surge de maneira natural em TQC pois há uma maneira natural de cobrir o espaço-tempo de Minkowski com um sistema de coordenadas. Conseqüentemente, há um conjunto de soluções natural para (2.3) através do qual expande-se o campo. Em particular, o espaço-tempo de Minkowski apresenta um campo de Killing tipo tempo, o campo $\left(\partial_{t}\right)^{a}$, e os modos são auto-vetores deste campo. Isto permite, no contexto de Mecânica Quântica, uma interpretação clara do conteúdo de energia das partículas. Todas estas características decorrem imediatamente do grupo de Poincaré e do princípio da covariância especial. 
Em espaços-tempos curvos, todavia, não há motivos para impormos qualquer tipo de simetria ao tensor métrico. Assim, vai por terra qualquer conceito natural de sistema de coordenadas e, conseqüentemente, de partículas. Nos casos especiais em que o espaçotempo guarda algum tipo de simetria, o campo de Killing associado pode ser usado para a construção de um sistema de coordenadas que pareça mais "natural". Mas mesmo estes sistemas de coordenadas, se existirem, não devem desempenhar o mesmo papel fundamental dos observadores de Minkowski em TQC. Este fato está intrinsecamente ligado ao princípio da covariância geral.

Como em TQCEC não há um sistemas de coordenadas privilegiado, não existe um conjunto privilegiado de modos normais através dos quais fazemos a expansão (2.32). Considere, então, um segundo conjunto de modos $\left\{\bar{u}_{j}(x)\right\}$ solução de (2.29). É absolutamente lícito expandirmos o campo em termos deste conjunto.

$$
\hat{\phi}(x)=\sum_{j}\left[\hat{\bar{a}}_{j} \bar{u}_{j}(x)+\hat{\bar{a}}_{j}^{\dagger} \bar{u}_{j}^{*}(x)\right]
$$

o operador $\hat{\phi}$ sendo o mesmo de antes, pois estamos lidando com um campo escalar. Esta decomposição define novos operadores de criação e destruição que, por sua vez, definem uma nova base para o espaço de configurações para o campo. Em particular, temos definido um novo estado de vácuo $|\overline{0}\rangle$ definido por

$$
\hat{\bar{a}}_{j}|\overline{0}\rangle=0, \quad \forall j .
$$

Ambos os conjuntos de modos, $\left\{u_{i}(x)\right\}$ e $\left\{\bar{u}_{j}(x)\right\}$, mais seus complexos conjugados, são completos. Logo, podemos expandir os modos barra em termos dos modos sem barra.

$$
\bar{u}_{j}(x)=\sum_{i}\left[\alpha_{j i} u_{i}(x)+\beta_{j i} u_{i}^{*}(x)\right]
$$

ou ainda expandir os modos sem barra em termos dos modos barra como

$$
u_{i}(x)=\sum_{j}\left[\alpha_{j i}^{*} \bar{u}_{j}(x)-\beta_{j i} \bar{u}_{j}^{*}(x)\right] .
$$

As relações (2.35) e (2.36) são chamadas de transformações de Bogoliubov. As matrizes $\alpha_{i j}$ e $\beta_{i j}$, conhecidas como coeficientes de Bogoliubov, são definidas através do produto interno (2.30) como segue.

$$
\alpha_{i j}=\left(\bar{u}_{i}, u_{j}\right), \quad \beta_{i j}=-\left(\bar{u}_{i}, u_{j}^{*}\right)
$$


Igualando (2.32) com (2.33), tomando o produto escalar com os modos sem barra e utilizando as definições (2.37), vem

$$
\hat{a}_{i}=\sum_{j}\left(\alpha_{j i} \hat{\bar{a}}_{j}+\beta_{j i}^{*} \hat{\bar{a}}_{j}^{\dagger}\right)
$$

Por outra, se tomarmos o produto escalar dos dois lados da igualdade com os modos barra, obtemos

$$
\hat{\bar{a}}_{j}=\sum_{i}\left(\alpha_{j i}^{*} \hat{a}_{i}-\beta_{j i}^{*} \hat{a}_{i}^{\dagger}\right)
$$

Da definição (2.37) decorrem as seguintes propriedades para os coeficientes de Bogoliubov:

$$
\begin{aligned}
\sum_{k}\left(\alpha_{i k} \alpha_{j k}^{*}-\beta_{i k} \beta_{j k}^{*}\right) & =\delta_{i j}, \\
\sum_{k}\left(\alpha_{i k} \beta_{j k}-\beta_{i k} \alpha_{j k}\right) & =0 .
\end{aligned}
$$

Segue das relações (2.38) e (2.39) que, quando $\beta_{i j} \neq 0$, os operadores $\hat{a}_{i}$ e $\hat{\bar{a}}_{i}$ não atuam da mesma forma sobre o espaço das configurações do campo. Em particular, estes espaços não compartilham do mesmo estado de vácuo, pois

$$
\hat{a}_{i}|\overline{0}\rangle=\sum_{j} \beta_{j i}^{*}\left|\overline{1}_{j}\right\rangle \neq 0
$$

se $\beta_{j i} \neq 0$, qualquer que seja $j$. Isto implica que o número de ocupação médio de um modo $u_{i}$ no estado de vácuo dos modos barra não será nulo, já que

$$
\left\langle\overline{0}\left|\hat{N}_{i}\right| \overline{0}\right\rangle=\sum_{j}\left|\beta_{j i}\right|^{2}
$$

Dizemos então que no vácuo dos modos $\bar{u}_{j}$ existem, em média, $\sum_{j}\left|\beta_{j i}\right|^{2}$ partículas dos $\operatorname{modos} u_{i}$.

Suponhamos que o espaço-tempo tenha um campo de Killing tipo tempo $\xi^{a}$. Se os modos $u_{i}$ forem de freqüência positiva com respeito ao campo de Killing, isto é, se

$$
£_{\xi} u_{i}=-\mathrm{i} \omega u_{i}
$$

e os modos $\bar{u}_{j}$ forem combinação de $u_{i}$ somente $\left(\operatorname{sem} u_{i}^{*}\right)$, então $\beta_{j i}=0$. Aqui $£_{\xi}$ é o operador derivada de Lie com respeito ao campo de Killing $\xi$. Se, por algum processo, os modos $\bar{u}_{j}$ tornarem-se uma mistura de modos sem barra de freqüência positiva e negativa, 
temos o que podemos chamar de produção de partículas se $\bar{u}_{j}$ puder, em alguma região, ser interpretado como sendo de freqüência positiva.

Destes resultados fica claro que o princípio da covariância geral implica que o conceito de partículas, em TQCEC, é desprovido de significado universal. Logo, em espaços-tempos arbitrários o conceito de partícula torna-se vago. Apesar disto, nos casos especiais em que o espaço-tempo apresenta uma região assintoticamente plana, o conceito de partícula ainda pode desempenhar algum papel.

Um exemplo bastante simples é o seguinte. Suponha um espaço-tempo que no infinito passado é plano e que evolui, no infinito futuro, para uma geometria também plana, passando por uma fase de expansão ou contração. Suponha que no passado os campos que descrevem a matéria estão em seu estado de vácuo. Nesta região, tudo se passa como em Minkowski e observadores inerciais não irão medir a presença de partículas com seus detectores. Chamaremos esta região de região in e o vácuo dos campos nesta região de estado de vácuo in; $|0 ; i n\rangle$. Assintoticamente no futuro, como a geometria é plana novamente, podemos definir novas famílias de observadores que constroem um novo estado de vácuo para os campos. Chamaremos esta região de região out e o vácuo dos campos nesta região de estado de vácuo out; $\mid 0 ;$ out $\rangle$. Conforme o espaço-tempo evolui, os modos in deixam de ter a forma de ondas planas que tinham no passado e passam a ser, em geral, funções mais complicadas. Logo, o que era onda plana com freqüência positiva no passado passa a ser uma combinações de freqüências positivas e negativas no futuro. Isto implica que $|0 ; i n\rangle$ não é mais o estado de nenhuma partículas para os observadores inerciais na região out. Para descobrir o conteúdo de partículas do sistema, estes observadores devem medir o valor de $\left\langle 0 ; i n\left|\hat{N}_{\text {out }}\right| 0 ; i n\right\rangle$, que para uma evolução arbitrária será não nulo. Conclui-se, então, que a evolução do espaço-tempo cria as partículas. É desta forma que o campo gravitacional cria pares.

Apesar de podermos nos valer do conceito de partículas no passado e assintoticamente no futuro, nada podemos afirmar sobre o conteúdo de partícula do sistema durante o processo de evolução. Esta situação é análoga à criação de pares em espaço plano. Mesmo em TQC, durante o processo de espalhamento não há sentido estrito no conceito individual de partícula, ele só surge muito longe da fonte espalhadora. 


\subsubsection{Efeito Hawking 7}

Considere uma estrela de massa $M$ esfericamente simétrica circundada por espaço vazio. Suponha que em algum momento, devido a sua evolução, este corpo colapse gravitacionalmente dando origem a um buraco negro. Por simplicidade, vamos impor que o colapso é esfericamente simétrico e que o buraco negro resultante tem carga e momentum angular nulos. Pelo fato de a implosão ser esfericamente simétrica, durante todo processo, a métrica externa à estrela e, depois, ao buraco negro é

$$
d s^{2}=-\left(1-\frac{2 M}{r}\right) d t^{2}+\left(1-\frac{2 M}{r}\right)^{-1} d r^{2}+r^{2}\left(d \theta^{2}+\operatorname{sen}^{2} \theta d \varphi^{2}\right)
$$

o que é garantido pelo teorema de Birkhoff. A divergência que aparece em (2.45) quando $r=2 M$ é fictícia; ela surge por conta da má escolha do sistema de coordenadas. A divergência em $r=0$, entretanto, não há como ser evitada.

A forma exata da métrica interna à estrela não nos interessa, posto que não estamos interessados nos detalhes do colapso. Sabemos, entretanto, que também ela deva ser esfericamente simétrica mas não pode ser estática. De forma bastante geral, podemos escrevê-la como

$$
d s^{2}=g_{\tau \tau}(\bar{r}, \tau) d \tau^{2}+g_{\bar{r} \bar{r}}(\bar{r}, \tau) d \bar{r}^{2}+2 g_{\tau \bar{r}}(\bar{r}, \tau) d \tau d \bar{r}+h^{2}(\bar{r}, \tau)\left(d \theta^{2}+\operatorname{sen}^{2} \theta d \varphi^{2}\right)
$$

onde $\tau$ é uma coordenada temporal no interior da estrela e $\bar{r}$ uma coordenada radial interna. A partir desta forma geral para o elemento de linha vamos construir um sistemas de coordenadas tal que a coordenada radial seja ortogonal à coordenada temporal. Isto pode ser obtido através de uma nova coordenada radial, função de $\bar{r}$ e $\tau$. Se impusermos, via lei de transformação, que neste novo sistema de coordenadas a componente da métrica cruzada entre tempo e coordenada radial seja nulo e que a componente puramente radial seja menos a componente puramente temporal vem condições sobre a transformação de coordenadas. Estas condições evidentemente dependem da métrica nas coordenadas antigas. Podemos ainda ajustar as constantes que comparecem na transformação de coordenadas para fixar que a coordenadas radial interna da superfície da estrela seja igual

\footnotetext{
${ }^{7}$ Para a derivação original de Hawking, ver [9]. Nesta seção, mostraremos uma derivação um pouco mais elaborada do resultado de Hawking do que a apresentada em [40.
} 
à sua coordenada radial externa. Assim, podemos reescrever a métria interna como

$$
d s^{2}=-A(r, \tau)\left(d \tau^{2}-d r^{2}\right)+\tilde{h}^{2}(r, \tau)\left(d \theta^{2}+\operatorname{sen}^{2} \theta d \varphi^{2}\right)
$$

onde a função $\tilde{h}$, para $r=r_{\text {estrela }}$, a coordenada radial da superfície da estrela, é tal que $\tilde{h}\left(r_{\text {estrela }}, \tau\right)=r_{\text {estrela }}$.

É conveniente reescrever as métricas interna e externa em termos, respectivamente, das coordenadas nulas $(U, V)$ e $(u, v)$. Vamos defini-las através das seguintes relações:

$$
\begin{array}{ll}
u=t-r^{*}, & v=t+r^{*}, \\
U=\tau-r, & V=\tau+r .
\end{array}
$$

Aqui, $r^{*}$ está relacionado com a coordenada $r$, fora da estrela, por

$$
d r^{*}=\left(1-\frac{2 M}{r}\right)^{-1} d r
$$

Nas coordenadas nulas, a métrica externa (2.45) torna-se

$$
d s^{2}=-C(r) d u d v+r^{2}\left(d \theta^{2}+\operatorname{sen}^{2} \theta d \varphi^{2}\right),
$$

com $C(r)=\left(1-\frac{2 M}{r}\right)$, enquanto que a interna (2.46) fica

$$
d s^{2}=-A(r, \tau) d U d V+\tilde{h}^{2}(r, \tau)\left(d \theta^{2}+\operatorname{sen}^{2} \theta d \varphi^{2}\right)
$$

Na superfície da estrela, podemos relacionar as coordenadas $(u, v)$ com as coordenadas $(U, V)$ através de

$$
U=a(u), \quad v=b(V),
$$

funções estas que são fixadas através da condição de continuidade da métrica sobre a superfície da estrela. Note que a relção entre $U$ e $u$ independe de $V$ ou $v$ e vice-versa. Isto, pois é razoável que a distorção dos cones de luz quando passamos do vácuo para o interior da estrela seja feita de forma contínua.

Neste espaço-tempo, considere um campo escalar $\hat{\phi}$ sem massa. O operador campo pode ser expresso por

$$
\hat{\phi}=\sum_{i}\left[f_{i} \hat{a}_{i}+f_{i}^{*} \hat{a}_{i}^{\dagger}\right]
$$




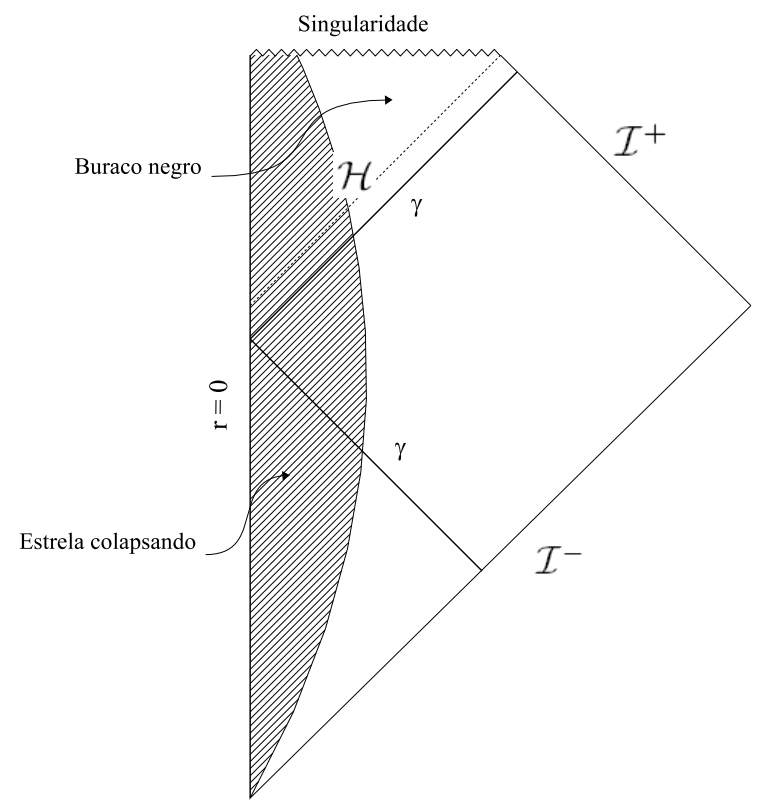

Figura 2.1: Diagrama de Penrose de um colapso estelar que dá origem a um buraco negro. O horizonte de eventos $\mathcal{H}$ é representado pela linha pontilhada e as linhas $\gamma$ representam a trajetória de um raio de luz na aproximação de ótica geométrica.

Os modos $\left\{f_{i}\right\}$ são solução de (2.29) na métrica de Schwarzschild quando $m=0$. Eles podem ser escolhidos no infinito passado nulo, $\mathcal{I}^{-}$, de forma tal que formem um conjunto completo de soluções satisfazendo as condições (2.31), onde a hiperfície $\Sigma$ é (arbitrariamente "próxima" a) $\mathcal{I}^{-}$, e que sejam de freqüência positiva com respeito ao campo de Killing tipo tempo $\left(\partial_{t}\right)^{a}$ sobre $\mathcal{I}^{-}$. Estes são os modos in, os que caminham em direção ao centro da estrela. Interpretamos $\hat{a}_{i}$ e $\hat{a}_{i}^{\dagger}$ como os operadores de destruição e criação de partículas no infinito passado nulo $\mathcal{I}^{-}$, ou seja, de partículas in. Com estes modos, a evolução do campo pode ser completamente determinada se fixarmos $\hat{\phi}$ e $\hat{\pi}$ sobre $\mathcal{I}^{-}$. Logo a expansão (2.52) é valida em qualquer lugar.

No futuro, depois de ocorrido o colapso, podemos determinar a evolução do campo se fixarmos $\hat{\phi}$ e $\hat{\pi}$ sobre o infinito futuro nulo $\mathcal{I}^{+}$e sobre o horizonte de eventos $\mathcal{H}$. Isto nos permite expandir o campo da seguinte forma:

$$
\hat{\phi}=\sum_{j}\left[p_{j} \hat{b}_{j}+p_{j}^{*} \hat{b}_{j}^{\dagger}+q_{j} \hat{c}_{j}+q_{j}^{*} \hat{c}_{j}^{\dagger}\right]
$$

O conjunto de modos $\left\{p_{j}\right\}$ são as soluções da equação de onda fixados por condições sobre 
$\mathcal{I}^{+}$, enquanto que os modos $\left\{q_{j}\right\}$ são aqueles fixados por condições sobre $\mathcal{H}$. Os modos $\left\{p_{j}\right\}$ e $\left\{q_{j}\right\}$ são conjuntos completos de funções que supomos satisfazerem as condições (2.31), respectivamente, sobre $\mathcal{I}^{+}$e $\mathcal{H}$. Além disto, impomos que $\left\{p_{j}\right\}$ sejam de freqüência positiva com respeito ao campo de Killing tipo tempo $\left(\partial_{t}\right)^{a}$ sobre $\mathcal{I}^{+}$. Estes são os modos out, aqueles que caminham em direção ao infinito futuro. Assim, interpretamos os operadores $\hat{b}_{j}$ e $\hat{b}_{j}^{\dagger}$ como os de destruição e criação de partículas em $\mathcal{I}^{+}$, isto é, de partículas out. Estes são os modos que nos interessam, pois desejamos saber o número de partículas no infinito futuro que tenham sido geradas pelo colapso. Sobre os modos $\left\{q_{j}\right\}$ não imporemos nenhuma restrição.

Vamos supor que o estado do campo no passado era $|0 ; i n\rangle$, o vetor tal que $\hat{a}_{i}|0 ; i n\rangle=0$, qualquer que seja $i$. Como $\left\{f_{i}\right\}$ forma um conjunto completo de funções em todo o espaçotempo, podemos expandir o conjunto $\left\{p_{j}\right\}$ em termos deles em $\mathcal{I}^{+}$,

$$
p_{j}=\sum_{i}\left[\alpha_{j i} f_{i}+\beta_{j i} f_{i}^{*}\right]
$$

A partir dos resultados da seção anterior, concluímos que o número de partículas que chegam aos observadores no infinito futuro é

$$
\left\langle 0 ; i n\left|\hat{b}_{j}^{\dagger} \hat{b}_{j}\right| 0 ; i n\right\rangle=\sum_{i}\left|\beta_{j i}\right|^{2}
$$

Vejamos como calcular estes coeficientes de Bogoliubov.

A equação para os modos na região exterior à estrela é

$$
\begin{aligned}
\partial_{t}^{2} \phi & -\left(1-\frac{2 M}{r}\right) \partial_{r}^{2} \phi-\left(1-\frac{2 M}{r}\right)\left(\frac{6 M-2 r}{r^{2}}\right) \partial_{r} \phi+ \\
& -\left(1-\frac{2 M}{r}\right) \frac{1}{r^{2}}\left[\frac{1}{\operatorname{sen} \theta} \partial_{\theta}\left(\operatorname{sen} \theta \partial_{\theta} \phi\right)+\frac{1}{\operatorname{sen}^{2} \theta} \partial_{\varphi}^{2} \phi\right]=0 .
\end{aligned}
$$

Desejamos que a solução seja oscilatória em $t$ e que seja regular e univocamente definida sobre uma casca esférica coberta pelas coordenadas angulares $(\theta, \phi)$. Isto implica que as soluções possíveis têm a seguinte forma:

$$
\phi^{ \pm}(r, \theta, \varphi, t)=\frac{1}{(2 \pi \omega)^{\frac{1}{2}}} e^{\mathrm{i} \omega t} \frac{F_{\omega l}^{ \pm}(r)}{r} e^{ \pm \mathrm{i} \omega r^{*}} \mathrm{Y}_{l m}(\theta, \varphi)
$$

onde $\mathrm{Y}_{l m}(\theta, \varphi)$ são os harmônicos esféricos e $F_{\omega l}^{ \pm}(r)$ funções da coordenada radial que 
obedece a seguinte equação:

$$
\begin{gathered}
\frac{1}{2}\left(1-\frac{2 M}{r}\right) \frac{d^{2}}{d r^{2}} F_{\omega l}^{ \pm}+\left(\frac{5 M-2 r}{r^{2}} \pm \mathrm{i} \omega\right) \frac{d}{d r} F_{\omega l}^{ \pm}+ \\
+\frac{1}{r}\left[\frac{l(l+1)}{2 r}-\frac{5 M-2 r}{r^{2}} \mp 2 \mathrm{i} \omega\right] F_{\omega l}^{ \pm}=0 .
\end{gathered}
$$

Logo, os modos in, no passado, e out, no futuro, respectivamente, são:

$$
\begin{aligned}
f_{\omega^{\prime} l m} & =\frac{1}{\left(2 \pi \omega^{\prime}\right)^{\frac{1}{2}}} e^{\mathrm{i} \omega^{\prime} v} \frac{F_{\omega^{\prime} l}^{+}}{r} \mathrm{Y}_{l m}, \\
p_{\omega l m} & =\frac{1}{(2 \pi \omega)^{\frac{1}{2}}} e^{\mathrm{i} \omega u} \frac{F_{\omega l}^{-}}{r} \mathrm{Y}_{l m} .
\end{aligned}
$$

O fato de a implosão da estrela ser esfericamente simétrica implica que a expansão (2.54) tem a seguinte forma:

$$
p_{\omega l m}=\int_{0}^{\infty} d \omega^{\prime}\left(\alpha_{\omega \omega^{\prime}} f_{\omega^{\prime} l m}+\beta_{\omega \omega^{\prime}} f_{\omega^{\prime} l m}^{*}\right)
$$

Para calcularmos os coeficientes da expansão (2.54), devemos conhecer a forma de $p_{\omega l m}$ em $\mathcal{I}^{-}$. Estes modos, ao serem propagados em direção ao passado a partir de $\mathcal{I}^{+}$, passam pelo interior da estrela. Quando o colapso toma parte, os modos são perturbados pela mudança na condição de contorno do campo gravitacional. Como resultado, teremos uma mistura de modos de freqüência positiva e negativa em $\mathcal{I}^{-}$. Ao ser propagada para trás, uma parte $p_{\omega l m}^{(1)}$ de $p_{\omega l m}$ é espalhada pelo potencial estático de Schwarzschild enquanto que a outra parte $p_{\omega l m}^{(2)}$ entra na estrela. A parte $p_{\omega l m}^{(1)}$ dá uma contribuição $\delta\left(\omega-\omega^{\prime}\right)$ ao coeficiente $\alpha_{\omega \omega^{\prime}}$. A parte $p_{\omega l m}^{(2)}$ será parte espalhada pelo campo no interior da estrela e parte transmitida para $\mathcal{I}^{-}$. A parte transmitida é a que codifica a criação de partículas. Por ora, vamos ignorar as partes espalhadas. Isto implica em aproximar os modos por raios de luz. Temos aqui uma aproximação da propagação dos modos pela ótica geométrica. Esta aproximação será tão melhor quanto menor o comprimento de onda dos modos $p_{\omega l m}$ em relação a $M$ quando eles cruzam a estrela. Veremos mais adiante que este é o caso.

Supondo continuidade dos modos ao entrarem no corpo que colapsa, temos que $p_{\omega l m}$ é proporcional a $e^{\mathrm{i} \omega u}$ fora e a $e^{\mathrm{i} \omega a^{-1}(U)}$ dentro da estrela. O fato de a trajetória do centro da estrela ser $U=V+$ cte, implica na seguinte condição para os modos:

$$
e^{\mathrm{i} \omega a^{-1}(U)}=e^{\mathrm{i} \omega a^{-1}(V+\text { cte })}, \quad \text { no centro. }
$$


Logo, do outro lado, já fora da estrela, os modos $p_{\omega l m}$ são proporcionais a $e^{\mathrm{i} \omega a^{-1}\left[b^{-1}(v)+\mathrm{cte}\right]}$. Temos, então, que determinar as funções $a$ e $b$ definidas em (2.51).

Pela condição de continuidade do campo gravitacional através da superfície da estrela, é possível relacionar as funções $a$ e $b$ com a métrica do espaço-tempo. Vamos supor que a implosão comece no instante $\tau=0$. Podemos descrever a evolução da superfície da estrela por

$$
r_{\text {estrela }}= \begin{cases}R_{0}, & \tau \leq 0 \\ R(\tau), & \tau>0\end{cases}
$$

Das definições (2.47), (2.48) e (2.51) decorre, sobre a superfície $S$ da estrela, que

$$
\begin{aligned}
& a^{\prime}(u)=\left.\frac{d U}{d u}\right|_{S}=\frac{C[R(\tau)][1-\dot{R}(\tau)]}{C[R(\tau)] \dot{t}(\tau)-\dot{R}(\tau)}, \\
& b^{\prime}(V)=\left.\frac{d v}{d V}\right|_{S}=\frac{C[R(\tau)] \dot{t}(\tau)+\dot{R}(\tau)}{C[R(\tau)][1+\dot{R}(\tau)]},
\end{aligned}
$$

onde o ponto sobre as coordenadas indica uma derivada em relação à coordenada temporal $\tau$. Pela condição de que geodésicas radiais tenham o mesmo comprimento sobre a superfície quando calculado tanto no sistema de coordenadas interno quanto no esterno, para $\tau>0$, vem que

$$
\dot{t}(\tau)=\frac{\left\{A[R(\tau), \tau] C[R(\tau)]\left[1-\dot{R}^{2}(\tau)\right]+\dot{R}^{2}(\tau)\right\}^{\frac{1}{2}}}{C[R(\tau)]} .
$$

Assim, substituindo (2.59) em (2.57) e (2.58), temos as formas de $a^{\prime}$ e de $b^{\prime}$ dadas por

$$
\begin{aligned}
a^{\prime}(u) & =\frac{C[R(\tau)][1-\dot{R}(\tau)]}{\left\{A[R(\tau), \tau] C[R(\tau)]\left[1-\dot{R}^{2}(\tau)\right]+\dot{R}^{2}(\tau)\right\}^{\frac{1}{2}}-\dot{R}(\tau)}, \\
b^{\prime}(V) & =\frac{\left\{A[R(\tau), \tau] C[R(\tau)]\left[1-\dot{R}^{2}(\tau)\right]+\dot{R}^{2}(\tau)\right\}^{\frac{1}{2}}+\dot{R}(\tau)}{C[R(\tau)][1+\dot{R}(\tau)]} .
\end{aligned}
$$

Desejamos saber qual é o número de partículas criado que chega ao infinito futuro. Os modos que carregam esta informação são aqueles que, depois do colapso, levam um tempo muito grande para atingir $\mathcal{I}^{+}$. Eles são tais que suas superfícies de fase constante são muito próximas ao horizonte de eventos, isto é, emergem da estrela pouco antes da sua formação. Os modos que "emergem" depois da formação do horizonte são aprisionados e têm como destino a singularidade. Os modos que emergem muito antes da formação do horizonte dão origem, no infinito, a um fluxo transiente de partículas que carregam 
informações sobre os detalhes do colapso; elas não nos interessam. Muito próximo do horizonte $(r=2 M)$, as coordenadas nulas $(u, v)$ crescem arbitrariamente. Isto implica que a oscilação dos modos, cujas superfícies de fase constante estão arbitrariamente próximas de $\mathcal{H}$, é muito alta. Estes modos, então, podem ser propagados sobre as superfícies de fase constante via ótica geométrica até $\mathcal{I}^{+}$. Somos levados a concluir que nos basta conhecer as funções $a$ e $b$ sobre a estrela em instantes imediatamente anteriores ao momento em que a superfície da estrela cruza o horizonte.

Como estamos interessados em $a$ e $b$ nesta região, podemos expandir $R(\tau)$ em uma séria de Taylor em torno de $\tau=\tau_{\mathcal{H}}$.

$$
R(\tau)=R_{\mathcal{H}}-\nu\left(\tau-\tau_{\mathcal{H}}\right)+\mathrm{O}\left[\left(\tau-\tau_{\mathcal{H}}\right)^{2}\right]
$$

onde $\nu=\left|\dot{R}\left(\tau_{\mathcal{H}}\right)\right|>0$, pois $\dot{R}(\tau)<0$, e $\tau_{\mathcal{H}}$ é o instante em que a superfície da estrela cruza o horizonte de eventos. Arbitrariamente próximo do horizonte, $C(r)$ fica arbitrariamente pequeno. Logo podemos escrevê-lo, muito próximo do horizonte, aproximadamente, como:

$$
C(r) \approx 2 \kappa\left(r-R_{\mathcal{H}}\right)
$$

onde $R_{\mathcal{H}}$ é a coordenada $r$ dos pontos sobre o horizonte de eventos e, para o caso de Schwarzschild, $\kappa=\frac{1}{4 M}$. Mantendo termos até primeira ordem em $\tau-\tau_{\mathcal{H}}$, vêm as seguintes expressões para $a^{\prime}$ e $b^{\prime}$ para instantes imediatamente anteriores à formação do horizonte:

$$
\begin{gathered}
a^{\prime}(u) \approx-\kappa\left(\tau-\tau_{\mathcal{H}}\right)(1+\nu), \\
b^{\prime}(V) \approx-\frac{A\left[R\left(\tau_{\mathcal{H}}\right), \tau_{\mathcal{H}}\right](1+\nu)}{2 \nu} \equiv \text { cte. }
\end{gathered}
$$

Utilizando a seguinte forma aproximada para a coordenada $\tau$ instantes antes da formação do horizonte,

$$
\tau \approx \frac{U+R_{\mathcal{H}}+\nu \tau_{\mathcal{H}}}{1+\nu}
$$

podemos integrar a equação para $a$ e obter a seguinte relação entre $U$ e $u$ sobre a superfície da estrela:

$$
a^{-1}(U)=-\frac{1}{\kappa} \ln \left(\frac{U-B_{1}}{B_{2}}\right) .
$$

Integrando a equação para $b$, vem que

$$
b^{-1}(v)=B_{3} v+B_{4},
$$


e destes resultados decorre que

$$
a^{-1}\left[b^{-1}(v)\right]=-\frac{1}{\kappa} \ln \left(\frac{v_{\mathcal{H}}-v}{D}\right),
$$

onde $B_{1}, B_{2}, B_{3}, B_{4}$ e $D$ são constantes de integração e $v_{\mathcal{H}}$ é a coordenada nula dos pontos do horizonte.

Com isto, e sabendo que podemos utilizar ótica geométrica para propagar os modos, temos que a forma de $p_{\omega l m}^{(2)}$ em $\mathcal{I}^{-}$, para $v<v_{\mathcal{H}}$, é aproximadamente dada por

$$
p_{\omega l m}^{(2)} \approx \frac{1}{(2 \pi \omega)^{\frac{1}{2}}} \frac{F_{\omega l}^{-}\left[r\left(v_{\mathcal{H}}\right)\right]}{r} \exp \left[-\mathrm{i} \frac{\omega}{\kappa} \ln \left(\frac{v_{\mathcal{H}}-v}{D}\right)\right],
$$

onde estimamos a função radial pelo seu valor sobre o horizonte de eventos no passado e omitimos uma fase constante. Por hora, vamos supor que não há espalhamento dos modos. Então $F_{\omega l}^{-}\left[r\left(v_{\mathcal{H}}\right)\right]=1$. Mais adiante comentaremos sobre esta questão. Para $v>v_{\mathcal{H}}$ temos que $p_{\omega l m}^{(2)}=0$, pois nesta região apenas há os modos $q_{j}$ definidos em (2.53). Para obter o coeficiente $\beta_{\omega \omega^{\prime}}$ de Bogoliubov basta calcularmos a transformada de Fourier sobre $\mathcal{I}^{-}$de $p_{\omega l m}^{(2)}$. Como os modos que contribuem para a produção de partículas têm $v$ pouco menor que $v_{\mathcal{H}}$, basta integrarmos no intervalo $\left[v_{\mathcal{H}}-\varepsilon, v_{\mathcal{H}}\right)$, com $\varepsilon$ um número real positivo pequeno. Entretanto, para que a integral tenha valor apreciável neste intervalo, faz-se necessário que $f_{\omega^{\prime} l m}$ oscile tanto quanto $p_{\omega l m}^{(2)}$. Isto é satisfeito se $\omega^{\prime} \varepsilon \gg 1$. Neste regime, a integral

$$
\beta_{\omega \omega^{\prime}} \approx-\frac{1}{2 \pi}\left(\frac{\omega^{\prime}}{\omega}\right)^{\frac{1}{2}} \int_{v_{\mathcal{H}}-\varepsilon}^{v_{\mathcal{H}}} d v\left(\frac{v_{\mathcal{H}}-v}{D}\right)^{-\mathrm{i} \frac{\omega}{\kappa}} e^{\mathrm{i} \omega^{\prime} v}
$$

volta o seguinte resultado:

$$
\beta_{\omega \omega^{\prime}} \approx-\frac{1}{2 \pi}\left(\frac{\omega^{\prime}}{\omega}\right)^{\frac{1}{2}} D^{i \frac{\omega}{\kappa}} e^{i \omega^{\prime} v_{\mathcal{H}}} \Gamma\left(1-i \frac{\omega}{\kappa}\right)\left(-\mathrm{i} \omega^{\prime}\right)^{-1+\mathrm{i} \frac{\omega}{\kappa}} .
$$

Tomando o módulo quadrado do resultado acima e utilizando as propriedades da função gama (ver [45]), obtemos

$$
\left|\beta_{\omega \omega^{\prime}}\right|^{2} \approx \frac{1}{2 \pi} \frac{1}{\kappa \omega^{\prime}} \frac{1}{e^{\frac{2 \pi \omega}{\kappa}}-1} .
$$

O número médio de partículas que chega ao infinito futuro com energias entre $\omega$ e $\omega+d \omega$ é dado por $d \omega \int_{0}^{\infty}\left|\beta_{\omega \omega^{\prime}}\right|^{2} d \omega^{\prime}$. Pelo fato de $\left|\beta_{\omega \omega^{\prime}}^{(2)}\right|^{2}$ ir com $\left(\omega^{\prime}\right)^{-1}$ para grandes valores de $\omega^{\prime}$, temos que a integral diverge. Esta divergência corresponde a uma taxa 
finita de produção de partículas que dura um tempo infinito. De fato, se considerarmos que as medidas são feitas durante um intervalo finito de tempo, as partículas serão bem descritas por pacotes de ondas centrados em $\omega$ e não por ondas planas com freqüência $\omega$.

O resultado (2.64) implica que a distribuição das partículas em energia é tal que um observador no infinito futuro entende que o buraco negro formado emite radiação como se fosse um corpo negro, caracterizado pela seguinte temperatura:

$$
T_{\mathrm{H}}=\frac{\kappa}{2 \pi k_{\mathrm{B}}}
$$

Para um buraco negro de Schwarzschild, a temperatura Hawking $T_{\mathrm{H}}$ vale $\frac{1}{8 \pi M k_{\mathrm{B}}}$. Este resultado, entretanto, é obtido desprezando-se o espalhamento dos modos. Se o espalhamento é levado em conta, $\left|F_{\omega l}^{-}\left[r\left(v_{\mathcal{H}}\right)\right]\right|^{2} \neq 1$ e somente uma fração dos modos que chegam até $\mathcal{H}$ são perturbados pelo colapso. Como este fator depende da freqüência dos modos, o espectro das partículas não é exatamente planckiano. Ainda assim, é dito que o espectro das partículas emitidas pelo buraco é térmico no seguinte sentido. Seja $\Gamma_{\omega}$ a fração de partículas emitidas em cada modo e $1-\Gamma_{\omega}$ a fração espalhada. Suponha que o buraco negro esteja imerso em um banho térmico cuja temperatura seja $T_{\mathrm{H}}$. Por conta do espalhamento, somente uma fração $\Gamma_{\omega}$ do banho é absorvido pelo buraco. Logo, na presença do banho, a taxa de emissão do buraco negro em cada modo independe de $\Gamma_{\omega}$ e passa a ser idêntica a de um corpo negro. Neste estágio, o buraco negro entra em equilíbrio térmico com o banho de radiação que o circunda.

A primeira vista é um tanto paradoxal que um buraco negro possa irradiar partículas, já que nada pode escapar do horizonte de eventos. Observando com mais atenção o resultado de Hawking, podemos concluir que o comprimento de onda médio das partículas emitidas é da ordem de $M$, da mesma ordem que o raio do buraco formado. Como não podemos localizar um quantum do campo em uma região igual a um comprimento de onda, não podemos dizer que as partículas são criadas em algum ponto do horizonte. Há, entretanto, dois argumentos heurísticos dados por Hawking para explicar o motivo pelo qual um buraco negro deveria irradiar. Os quanta, pelo fato de tentarmos localizá-los no interior de um buraco negro cujo tamanho é comparável a seu comprimento de onda típico, têm grande probabilidade de tunelarem pelo horizonte de eventos. Alternativamente, a criação de pares próximos ao buraco poderia explicar o efeito Hawking. Pares virtuais 
que vivem próximos ao horizonte e têm comprimento de onda da ordem de $M$ estão tipicamente separados por $M$ e por isto sentem uma grande força de maré. Esta força é intensa o suficiente para não permitir a recombinação dos pares. Uma vez separadas, as partículas com energia positiva escapam para o infinito enquanto que as partículas de energia negativa são engolidas pelo buraco negro.

Apesar de nas imediações do horizonte o conceito de partículas ser mal definido, é claro, para os observadores no infinito futuro, que as partículas criadas carregam energia para $\mathcal{I}^{+}$. A única fonte de energia possível para estas partículas seria o campo gravitacional da estrela. Logo, podemos concluir que com o tempo a massa do buraco diminui. Este é o problema do back-reaction. Não se sabe se buracos negros seriam capazes de evaporarem até sumirem completamente. Como a temperatura vai com o inverso da massa, quanto mais massa o buraco perde maior a energia típica das partículas que emite e, assim, mais rapidamente ele perde massa. Mas o estágio final, quando sua massa atinge a escala de Planck, só pode ser compreendido no contexto de uma teoria quântica da gravidade.

Outra questão interessante que emerge do efeito Hawking é sobre a natureza dos modos in. O processo de criação de partículas via colapso gravitacional é protagonizado por dois efeitos: o campo gravitacional variável no tempo e o desvio para o vermelho gravitacional líquido. O primeiro efeito é imprescindível, pois é ele que gera a mistura dos modos de freqüência positiva e negativa no infinito futuro. O segundo, muito intenso no caso de um colapso gerando um buraco negro, modifica a freqüência dos modos in que chegam ao infinito futuro. É em decorrência deste segundo efeito que é imperativo que os modos in tenham freqüência arbitrariamente alta para que a integral (2.63) tenha uma contribuição apreciável. Ao mergulharem no corpo que colapsa, os modos in sofrem um desvio para o azul. Aqueles que emergem imediatamente antes da formação do horizonte percebem um campo muito mais intenso do que aquele que experimentaram ao mergulhar. Assim, o desvio para o vermelho experimentado por estes modos, ao subirem em direção ao infinito futuro, é brutalmente maior que o desvio para o azul experimentado anteriormente. Isto gera um desvio para o vermelho líquido. A diferença entre os desvios para o azul e para o vermelho é tão grande que é necessário que os modos in tenham freqüência arbitrariamente alta no passado para chegarem com freqüência finita no futuro. Freqüências muito altas 
estão intrinsecamente ligadas a comprimentos de onda arbitrariamente curtos, inclusive aqueles abaixo da escala de Planck. Apesar de ser uma extrapolação do cálculo de TQCEC realizado acima, não esperamos que TQC e RG sejam válidas nas escalas de tempo e espaço que os modos in experimentam no passado. Seriam os buracos negros grandes microscópios da física trans-planckiana? A física na escala de Planck modifica a forma do espectro térmico previsto por Hawking ou não? Estas são perguntas que ainda hoje são motivo de pesquisa. 


\section{Capítulo 3}

\section{Analogia em fluidos}

Neste capítulo apresentaremos a analogia que há entre um campo escalar não-maciço submetido a ação de um campo gravitacional e o som que se propaga em um fluido cujo fluxo é inomogêneo. As propriedades e as definições que mostraremos nas próxima seções independem das equações que ditam a dinâmica do meio e se aplicam a outros contextos. Escolhemos o modelo do fluido, pois este fornece a analogia conceitualmente mais simples que pode ser feita entre a matéria condensada e a RG.

Na seção 3.1 construiremos a equação para perturbações lineares sobre o fluxo irrotacional de um fluido ideal e barotrópico. Estas perturbações sobre o fluxo de fundo interpretaremos como som. Mostraremos que o som em um fluido, cujo fluxo é inomogêneo, pode ser descrito através de um campo de Klein-Gordon não maciço definido sobre uma geometria curva efetiva. Esta geometria efetiva será entendida como um espaço-tempo especial somente provado pelo som.

Na seção 3.2 utilizaremos técnicas e terminologias da RG para expor algumas propriedades gerais dos espaços-tempos acústicos. Mostraremos que a métrica acústica se encaixa na classe de métricas lorentzianas e que os espaços-tempos acústicos são causalmente estáveis. Estas são propriedades importantes para que a analogia seja bem posta. Já na seção 3.3 exibiremos como definir os conceitos de ergoregião, horizonte de eventos e gravidade superficial no contexto de análogos. Eles são importantes, pois são análogos àqueles que caracterizam buracos negros em RG.

Iremos além da hipótese de um fluido ideal na seção 3.4. O som em um fluido viscoso 
serve para ilustrar a faceta mais sedutora dos análogos. Mostraremos que a manifestação da estrutura microscópica do fluido através da viscosidade pode quebrar a invariância de Lorentz. Esta quebra é relevante somente quando estamos abaixo da escala de Planck do modelo, isto é, na escala em que a descrição do fluido como contínuo perde sentido. Na seção 3.5 tocaremos em algumas questões relacionadas à implementação experimental do modelo do fluido.

\subsection{Som em fluidos ideais}

\subsubsection{Equações para o fluido}

Considere um fluido ideal. Por definição, um fluido ideal é aquele no qual o transporte de momentum e energia se dá através da pressão e do fluxo de massa. Para esta classe de fluidos, a segunda lei de Newton e a hipótese de conservação de massa implicam, respectivamente, nas seguintes equações:

$$
\begin{gathered}
\partial_{t} \mathbf{v}+(\mathbf{v} \cdot \nabla) \mathbf{v}+\frac{\nabla P}{\rho}=0 \\
\partial_{t} \rho+\nabla \cdot(\rho \mathbf{v})=0
\end{gathered}
$$

na ausência de forças externas e de fontes e sorvedouros de matéria 1 . A equação (3.1) é a equação de Euler e a (3.2) é a equação da continuidade para a massa. Aqui v(x,t), $\rho(\mathbf{x}, t)$ e $P(\mathbf{x}, t)$ são, nesta ordem, o campo de velocidades, a densidade de massa e a pressão no ponto $\mathrm{x}$ do fluido no instante $t$. As equações (3.1) e (3.2) formam um sistema insuficiente para determinar estas cinco funções. Necessitamos, então, de uma equação que relacione $P$ e $\rho$, isto é, uma equação de estado.

Pela primeira lei da Termodinâmica, temos

$$
d u=T d s-P d(1 / \rho),
$$

onde $u$ é a energia e $s$ a entropia, ambas por unidade de massa. Daí obtemos que

$$
P(s, \rho)=\rho^{2}\left(\frac{\partial u}{\partial \rho}\right)_{s},
$$

\footnotetext{
${ }^{1} \mathrm{~A}$ descrição relativística do fluido nos leva essencialmente aos mesmos resultados que mostraremos neste capítulo. Ver referência [46].
} 
se considerarmos $s$ e $\rho$ como variáveis independentes. Conhecendo a função $u(s, \rho)$, temos uma relação entre $P, \rho$ e $s$ a partir de (3.3) . A energia interna pode ser obtida via métodos de mecânica estatística ou empiricamente. A hipótese de idealidade implica na equação que falta. Segundo ela

$$
\frac{d s}{d t}=\partial_{t} s+(\mathbf{v} \cdot \nabla) s=0
$$

O sistema de equações (3.1), (3.2), (3.3) e (3.4) é completo. Com este sistema, mais condições iniciais e de contorno, podemos determinar as funções $\mathbf{v}(\mathbf{x}, t), \rho(\mathbf{x}, t), P(\mathbf{x}, t)$ e $s(\mathbf{x}, t)$. A partir deste ponto, vamos supor que o fluido em questão é isentrópico, isto é, que em qualquer instante a entropia tem o mesmo valor em todos os pontos do fluido. Neste caso, a equação (3.4) é identicamente satisfeita. Então, com o sistema formado pela equações (3.1), (3.2) e (3.3), mais condições iniciais e de contorno, somos capazes de determinar as funções $\mathbf{v}(\mathbf{x}, t), \rho(\mathbf{x}, t)$ e $P(\mathbf{x}, t)$. A condição de isentropicidade implica que o fluido é barotrópico. Um fluido barotrópico é aquele cuja pressão depende somente da densidade de matéria.

Impondo que o fluxo seja irrotacional, isto é, que $\nabla \times \mathbf{v}=0 \sqrt{2}$, podemos escrever o fluxo $\mathbf{v}$ do fluido em termos de uma função escalar $\psi$, relacionada ao fluxo por

$$
\mathbf{v}=-\nabla \psi
$$

Esta definição, quando substituída em (3.1), nos permite integrar uma vez a equação de Euler se utilizarmos

$$
\nabla u=\frac{P}{\rho^{2}} \nabla \rho
$$

Este equação segue da primeira lei da termodinâmica mais a hipótese de isentropicidade. Por fim, temos o seguinte sistema:

$$
\left\{\begin{array}{l}
-\rho \partial_{t} \psi+\frac{\rho}{2}(\nabla \psi)^{2}+P+\rho u=0 \\
\partial_{t} \rho-\nabla \cdot(\rho \nabla \psi)=0, \\
u=u(\rho)
\end{array}\right.
$$

\footnotetext{
${ }^{2}$ Para a dedução da equação para o som em um fluido cujo fluxo não é irrotacional, ver [4]. Não abordaremos esta questão aqui, pois fluxos com vorticidade não nula não são desejados no contexto de análogos.
} 
mais a relação (3.3), para determinar as funções $\psi, \rho$ e $P$. A densidade de lagrangeana $\mathcal{L}$ que implica no sistema (3.6) é

$$
\mathcal{L}=\rho \partial_{t} \psi-\frac{1}{2} \rho(\nabla \psi)^{2}-\rho u(\rho)
$$

a menos de uma constante multiplicativa e termos de fronteira.

\subsubsection{Equação para o som}

Desejamos descrever perturbações sobre o fluxo de fundo. Para tanto, considere um estado do fluido descrito por $\psi$ e $\rho$ tal que possa ser escrito em termos de uma configuração de fundo $\psi_{0}$ e $\rho_{0}$ mais perturbações.

$$
\begin{gathered}
\psi=\psi_{0}+\psi_{1}, \\
\rho=\rho_{0}+\rho_{1} .
\end{gathered}
$$

Suponha que as perturbações $\psi_{1}$ e $\rho_{1}$ sejam pequenas frente à configuração de fundo. Com "pequeno" queremos dizer que a maior parte do transporte de energia e momentum no fluido se dá através do fluxo de fundo. Substituindo a forma de $\psi$ e $\rho$ no sistema (3.6), guardando somente termos até primeira ordem nas perturbações e tomando que $\psi_{0}$ e $\rho_{0}$ são soluções conhecidas do sistema (3.6), vem

$$
\left\{\begin{array}{l}
\rho_{0}\left(-\partial_{t} \psi_{1}+\nabla \psi_{0} \cdot \nabla \psi_{1}\right)+\rho_{1}\left(\frac{d P}{d \rho}\right)_{\rho=\rho_{0}}=0 \\
\partial_{t} \rho_{1}-\nabla \cdot\left(\rho_{0} \nabla \psi_{1}+\rho_{1} \nabla \psi_{0}\right)=0 .
\end{array}\right.
$$

Isolando $\rho_{1}$ na primeira, substituindo na segunda e utilizando a definição

$$
c^{2}(\mathbf{x}, t)=\left(\frac{d P}{d \rho}\right)_{\rho=\rho_{0}}
$$

obtemos uma equação para a perturbação $\psi_{1}$,

$$
-\partial_{t}\left[\frac{\rho_{0}}{c^{2}}\left(\partial_{t} \psi_{1}+\mathbf{v}_{0} \cdot \nabla \psi_{1}\right)\right]+\nabla \cdot\left[\rho_{0} \nabla \psi_{1}+\frac{\rho_{0}}{c^{2}}\left(\partial_{t} \psi_{1}+\mathbf{v}_{0} \cdot \nabla \psi_{1}\right) \mathbf{v}_{0}\right]=0
$$

No caso particular em que $\mathbf{v}_{0}=\mathbf{0}$ e $\rho_{0} \equiv$ cte, segue de (3.10) a equação de D'Alembert,

$$
\frac{1}{c^{2}} \partial_{t}^{2} \psi_{1}-\nabla^{2} \psi_{1}=0 .
$$


Neste caso particular, temos que $c \equiv$ cte é a velocidade de fase das ondas. Se tomarmos menos o gradiente da equação acima, obtemos uma equação de ondas para a perturbação $\mathbf{v}_{1}$. Logo, os elementos do fluido ondulam.

Vibrações na estrutura do meio material em que estamos imersos e que perturbam a estrutura do aparelho auditivo, sendo traduzidas como sensações pelo cérebro: a esta experiência cotidiana atribuímos o nome de som. Via de regra, estas vibrações são produzidas por alguma fonte. Esta fonte perturba o meio ao redor, causando pequenas variações na densidade. A estas variações na densidade correspondem variações na pressão (ou nas tensões, no caso de sólidos). Estas mudanças locais na pressão implicam no movimento das partículas constituintes do meio. Os deslocamentos de matéria causam novas variações na densidade, e assim o som se propaga até nós. Isto nos permite entender $\psi_{1}$ como o campo que descreve o som. A função $c(\mathbf{x}, t)$ é a velocidade do som relativa ao meio material.

\subsubsection{Analogia com a RG}

Vamos mostrar como surge a analogia entre a equação (3.10) e um campo escalar não maciço que se propaga em uma geometria curva. Para tal fim, devemos reescrever (3.10) de maneira apropriada. Considere as seguintes quantidades auxiliares:

$$
\begin{gathered}
V_{t}=-\frac{\rho_{0}}{c^{2}}\left(\partial_{t} \psi_{1}+\mathbf{v}_{0} \cdot \nabla \psi_{1}\right) \\
\mathbf{V}=\rho_{0} \nabla \psi_{1}+\frac{\rho_{0}}{c^{2}}\left(\partial_{t} \psi_{1}+\mathbf{v}_{0} \cdot \nabla \psi_{1}\right) \mathbf{v}_{0}
\end{gathered}
$$

Por meio delas, podemos reescrever a equação (3.10) como

$$
\partial_{t} V_{t}+\nabla \cdot \mathbf{V}=0
$$

A matriz coluna, cujas componentes são $V_{t}$ e $\mathbf{V}$, está relacionada com $\partial_{t} \psi_{1}$ e $\nabla \psi_{1}$ através do seguinte produto de matrizes:

$$
\left[\begin{array}{c}
V_{t} \\
V_{x} \\
V_{y} \\
V_{z}
\end{array}\right]=\frac{\rho_{0}}{c^{2}}\left[\begin{array}{cccc}
-1 & -\mathrm{v}_{0 x} & -\mathrm{v}_{0 y} & -\mathrm{v}_{0 z} \\
-\mathrm{v}_{0 x} & c^{2}-\mathrm{v}_{0 x}^{2} & -\mathrm{v}_{0 x} \mathrm{v}_{0 y} & -\mathrm{v}_{0 x} \mathrm{v}_{0 z} \\
-\mathrm{v}_{0 y} & -\mathrm{v}_{0 y} \mathrm{v}_{0 x} & c^{2}-\mathrm{v}_{0 y}^{2} & -\mathrm{v}_{0 y} \mathrm{v}_{0 z} \\
-\mathrm{v}_{0 z} & -\mathrm{v}_{0 z} \mathrm{v}_{0 x} & -\mathrm{v}_{0 z} \mathrm{v}_{0 y} & c^{2}-\mathrm{v}_{0 z}^{2}
\end{array}\right]\left[\begin{array}{c}
\partial_{t} \psi_{1} \\
\partial_{x} \psi_{1} \\
\partial_{y} \psi_{1} \\
\partial_{z} \psi_{1}
\end{array}\right]
$$


Em termos dele, a equação (3.10) fica

$$
\partial_{\mu}\left(f^{\mu \nu} \partial_{\nu} \psi_{1}\right)=0
$$

onde $f^{\mu \nu}$ são as componentes da seguinte matriz:

$$
f^{\mu \nu}=\frac{\rho_{0}}{c^{2}}\left[\begin{array}{ccc}
-1 & \vdots & -\mathrm{v}_{0 i} \\
\ldots \ldots & . & \ldots \ldots \ldots \ldots \ldots \\
-\mathrm{v}_{0 j} & \vdots & c^{2} \delta_{i j}-\mathrm{v}_{0 i} \mathrm{v}_{0 j}
\end{array}\right] .
$$

Através da matriz $f^{\mu \nu}$ podemos construir uma outra, a matriz $g^{\mu \nu}$, relacionada com a primeira por $f^{\mu \nu}=\sqrt{-g} g^{\mu \nu}$. Aqui, $g$ é o determinante da matriz inversa de $g^{\mu \nu}$. Ela será denotada por $g_{\mu \nu}$. Sabendo que

$$
g=\operatorname{det}\left\{f^{\mu \nu}\right\}, \quad \operatorname{det}\left\{f^{\mu \nu}\right\}=-\frac{\rho_{0}^{4}}{c^{2}}
$$

obtemos a forma das matrizes $g^{\mu \nu}$ e $g_{\mu \nu}$,

$$
\begin{gathered}
g^{\mu \nu}=\frac{1}{\rho_{0} c}\left[\begin{array}{ccc}
-1 & \vdots & -\mathrm{v}_{0 i} \\
\ldots \ldots & . & \ldots \ldots \ldots \ldots \ldots \\
-\mathrm{v}_{0 j} & \vdots & c^{2} \delta_{i j}-\mathrm{v}_{0 i} \mathrm{v}_{0 j}
\end{array}\right], \\
g_{\mu \nu}=\frac{\rho_{0}}{c}\left[\begin{array}{ccc}
-\left(c^{2}-\mathrm{v}_{0}^{2}\right) & \vdots & -\mathrm{v}_{0 i} \\
\ldots \ldots \ldots \ldots & . & \ldots \ldots \\
-\mathrm{v}_{0 j} & \vdots & \delta_{i j}
\end{array}\right] .
\end{gathered}
$$

Interpretando (3.12) como as componentes de um tensor métrico, o elemento de linha associado é:

$$
d s^{2}=\frac{\rho_{0}}{c}\left[-\left(c^{2}-\mathrm{v}_{0}^{2}\right) d t^{2}-2 \mathbf{v}_{0} \cdot d \mathbf{x} d t+d \mathbf{x} \cdot d \mathbf{x}\right]
$$

Por fim, ao dividirmos por $\sqrt{-g}$, vem a seguinte forma para a equação (3.10):

$$
\frac{1}{\sqrt{-g}} \partial_{\mu}\left(\sqrt{-g} g^{\mu \nu} \partial_{\nu} \psi_{1}\right)=0
$$

A operador que aparece na equação (3.14) atuando sobre $\psi_{1}$ é o operador de LaplaceBeltrami, quando escrito no sistema de coordenadas no qual a métrica tem componentes $g_{\mu \nu}$. A equação (3.14) é exatamente a equação (2.29), quando $m=0$. É neste ponto que reside a analogia entre a $R G$ e a acústica em fluidos. A equação (3.14) nos diz, então, 
que o som percebe as inomogeneidades do fluxo do fluido como um espaço-tempo curvo efetivo. Ao espaço-tempo emergente dá-se o nome acústico e ao seu tensor métrico o nome de métrica acústica. Para termos esperança de simularmos campos gravitacionais através dos espaços-tempos acústicos, é necessário que a métrica acústica tenha pelo menos algumas propriedades das métricas que aparecem na RG. Na seção 3.2 mostraremos que de fato a métrica (3.12) tem características que nos permitem simular espaços-tempos da RG.

Para não sobrecarregar a notação, daqui em diante evitaremos usar os sub-índices que indicam soluções de fundo ou perturbações. Usaremos tais sub-índices somente quando forem indispensáveis para evitar algum tipo de confusão.

\subsubsection{Espaço-tempo newtoniano versus espaço-tempo efetivo}

As equações (3.1) e (3.2) descrevem um fluido ideal newtoniano, isto é, um fluido ideal cujo escoamento dá-se a uma velocidade muito menor que a da luz. O espaço-tempo de fundo do laboratório é, em excelente aproximação, aquele da mecânica de Newton: um espaço tridimensional euclidiano mais um tempo universal. As medidas das propriedades do fluido tomadas por algum observador inercial são funções escalares definidas sobre este espaço-tempo. Da Relatividade Restrita (RR), sabemos que a estrutura causal do espaço-tempo plano é dada pela métrica Minkowski; esta é a estrutura causal percebida no referencial do laboratório.

O som, por sua vez, tem um ponto de vista radicalmente diferente. Segundo a equação (3.14), o som propagando-se sobre um fluxo inomogêneo acopla-se com a métrica (3.12). Suponha hipotéticos observadores imersos no fluido que só pudessem provar ou se comunicar através de sinais sonoros. Para eles, tudo se passaria como se estivessem sob a ação de um campo gravitacional descrito pela métrica acústica. Eles, então, perceberiam uma estrutura causal totalmente diferente daquela do laboratório.

É notável que as equações da mecânica newtoniana para o fluido impliquem em uma equação para perturbações lineares sobre o fluxo de fundo que seja covariante por transformações arbitrárias de coordenadas. Assim como na RG, não existe uma maneira natural de cobrir os espaços-tempos acústicos com um sistema de coordenadas baseada na 
sua métrica acústica. Entretanto, diferentemente da RG, estes espaços-tempos emergem de um espaço-tempo mais fundamental, no sentido que serve de pano de fundo para todos os fenômenos físicos que ocorrem no laboratório. Isto implica que os espaços-tempos acústicos já nascem cobertos por algum sistema de coordenadas, no caso, aquele definidos por observadores inerciais em repouso em relação ao laboratório.

É desta forma que se constrói a analogia entre a acústica em fluidos e a RG. Utilizamos a métrica definida a partir de (3.10) para reescrever a estrutura causal do espaço-tempo do laboratório. Esta estrutura que emerge das inomogeneidades do fluxo do fluido é somente percebida por hipotéticos observadores que se comunicam através do som. A relação entre a RG e a acústica dá-se, então, somente do ponto de vista cinemático. Não podemos censurar perguntas como "Ok, se estamos sob a ação de um campo gravitacional, quem dita sua dinâmica?" por parte destes observadores hipotéticos. Para perguntas deste tipo a resposta seria "Ora, olhem para as equações da hidrodinâmica.". É a hidrodinâmica, e não as equações de Einstein, que ditam a evolução das componentes da métrica efetiva e, conseqüentemente, do espaço-tempo acústico. Logo, somos somente capazes de mimicar características da $\mathrm{RG}$ que independam das equações que ditam a dinâmica do campo gravitacional. Veremos mais adiante que isto é suficiente para o propósito de simular TQCEC em sistemas de matéria condensada.

\subsection{Espaços-tempos acústicos: algumas propriedades}

\subsubsection{Signatura da métrica acústica}

A signatura de um tensor métrico é definida como o número de autovalores com sinais positivos ou negativos da matriz correspondente em alguma base. Como os autovalores de uma matriz independem da base escolhida, a signatura é uma propriedade intrínseca à métrica. Os tensores métricos que aparecem na RG pertencem à classe das métricas lorentzianas: métricas cuja signatura é $(-,+,+,+)$ ou $(+,-,-,-)$, dependendo a convenção. 
Logo, para que seja possível simularmos espaços-tempos da RG é indispensável que a métrica acústica seja lorentziana. Ao diagonalizarmos (3.12) obtemos os seguintes autovalores:

$$
\begin{aligned}
& \lambda_{1}=1>0, \\
& \lambda_{2}=1>0, \\
& \lambda_{3}=\frac{1}{2}\left[\sqrt{\left(c^{2}-\mathrm{v}^{2}-1\right)^{2}+4 c^{2}}-\left(c^{2}-\mathrm{v}^{2}-1\right)\right]>0, \\
& \lambda_{4}=-\frac{1}{2}\left[\sqrt{\left(c^{2}-\mathrm{v}^{2}-1\right)^{2}+4 c^{2}}+\left(c^{2}-\mathrm{v}^{2}-1\right)\right]<0 .
\end{aligned}
$$

Portanto, as métricas acústicas se enquadram na classe das métricas lorentzianas.

\subsubsection{4-velocidade e 4-aceleração}

Quando parametrizada pelo tempo $t$ do laboratório, a linha de mundo de um observador é

$$
x^{\mu}(t)=(t, x(t), y(t), z(t)) .
$$

Nesta parametrização, o 4-vetor tangente à curva é

$$
v^{\mu}=\frac{d x^{\mu}(t)}{d t}=\left(1, v_{x}, v_{y}, v_{z}\right)=(1 ; \boldsymbol{v})
$$

cujo módulo quadrado, em relação à métrica acústica, é dado por

$$
g_{\mu \nu} v^{\mu} v^{\nu}=-\frac{\rho}{c}\left[c^{2}-(\mathbf{v}-\boldsymbol{v})^{2}\right]
$$

O parâmetro afim da curva é definido por

$$
\tau(t)=\int^{t} \sqrt{g_{\mu \nu} v^{\mu} v^{\nu}} d t^{\prime}
$$

No contexto da RG, o parâmetro afim é interpretado como o tempo próprio do observador que segue aquela linha de mundo. Em espaços-tempos acústicos não há nenhuma interpretação física para este parâmetro. Em geometria diferencial, o parâmetro afim é o comprimento da curva; uma curva parametrizada pelo seu parâmetro afim é dita estar parametrizada pelo comprimento de arco. A 4-velocidade é definida como o 4-vetor tangente à curva nesta parametrização,

$$
u^{\mu}=\frac{d x^{\mu}(\tau)}{d \tau}
$$


Esta parametrização implica que

$$
g_{\mu \nu} u^{\mu} u^{\nu}=-1
$$

Para um elemento de fluido, a relação entre o parâmetro afim de sua linha de mundo e o tempo do laboratório é

$$
d \tau=\sqrt{\rho c} d t
$$

Assim, a 4-velocidade do fluido pode ser expressa como

$$
\mathrm{u}^{\mu}=\frac{1}{\sqrt{\rho c}}(1 ; \mathbf{v})
$$

que pode ser escrita em termos da derivada covariante através de

$$
\mathrm{u}^{\mu}=-\sqrt{\rho c} \nabla^{\mu} t
$$

A 4-aceleração de um observador é definida por

$$
a^{\mu}=u^{\sigma} \nabla_{\sigma} u^{\mu}
$$

Ela nos dá uma medida de quanto um observador desvia-se da geodésica que seguiria caso fosse "livre numa geometria dada por $g_{\mu \nu}$ ". A partir desta definição, podemos descobrir qual a equação para a linha de mundo dos elementos do fluido no espaço-tempo acústico. Temos

$$
\mathrm{a}^{\mu}=\mathrm{u}^{\sigma} \nabla_{\sigma} \mathrm{u}^{\mu}=\sqrt{\rho c} \nabla^{\sigma} t \nabla_{\sigma}\left(\sqrt{\rho c} \nabla^{\mu} t\right)=\left\{\begin{array}{ccc}
0 & \text { se } & \mu=0, \\
\frac{\partial_{j}(\rho c)}{2 \rho^{2}} & \text { se } & \mu=j .
\end{array}\right.
$$

Logo, a 4-aceleração dos elementos de fluido é

$$
\mathrm{a}^{\mu}=\frac{1}{2 \rho^{2}}(0 ; \nabla(\rho c))
$$

Este resultado implica que os elementos de fluido somente seguirão geodésicas do espaçotempo acústico quando a equação de estado for tal que o produto entre a densidade e a velocidade do som não depende da posição. Isto é um tanto inesperado, haja visto que é o fluxo dos elementos de fluido que define o espaço-tempo acústico. Por isto, ingenuamente poderia-se imaginar que o próprio fluxo de fundo do fluido sempre seguiria uma geodésica, o que não é verdade. 


\subsubsection{Estabilidade causal}

Um espaço-tempo dito causalmente estável é aquele que não apresenta curvas fechadas tipo tempo. Esta propriedade impede, por exemplo, as fantásticas viagens no tempo. Há um teorema que garante que o espaço-tempo $\left(M, g_{a b}\right)$ é causalmente estável se, e somente se, sobre $M$ existe uma função diferenciável $f$ tal que o campo vetorial $\nabla^{a} f$ é tipo tempo e direcionado para o passado em todo ponto 3

Em espaços-tempos acústicos, o papel da função $f$ pode muito bem ser desempenhado pelo parâmetro temporal $t$ do laboratório. O campo $\nabla^{a} t=-\frac{1}{\rho c}(1 ; \mathbf{v})$ é tipo tempo,

$$
\nabla^{a} t \nabla_{a} t=-\frac{1}{\rho c}<0
$$

e é voltado ao passado em todo lugar. Pela relação (3.16), vemos que este campo está ligado à 4-velocidade dos elementos do fluido. É interessante reparar que a presença deste fluxo de fundo no referencial do laboratório evita problemas de causalidade que podem surgir caso o espaço-tempo não seja causalmente estável.

\subsubsection{Geodésicas tipo "som"}

O limite da acústica geométrica em um meio não-homogêneo faz sentido quando as escalas de tempo e comprimento das inomogeneidades do fluxo de fundo são muito maiores quando comparadas à freqüência e ao comprimento de onda típicos da perturbação sonora. Quando isto é satisfeito, podemos desprezar a natureza ondulatória do som e passar a descrevê-lo através de raios cujos vetores tangentes apontam na mesma direção da propagação da onda sonora. Esta descrição é possível pois, neste regime, as inomogeneidades afetam pouco a direção de propagação das ondas. Isto decorre do fato de as ondas serem muito pouco espalhadas pelo meio.

Vamos tomar o potencial do campo de velocidades linearizado como o produto entre uma amplitude e uma fase, $\psi(\mathbf{x}, t)=A(\mathbf{x}, t) e^{\mathrm{i} \phi(\mathbf{x}, t)}$. Substituindo esta forma para $\psi$ em (3.14) vem

$$
g^{\mu \nu} \partial_{\mu} \phi \partial_{\nu} \phi-\mathrm{i} g^{\mu \nu} \partial_{\mu} \partial_{\nu} \phi=0
$$

\footnotetext{
${ }^{3}$ Ver referência [36, capítulo 8 teorema 8.2.2.
} 
onde tomamos a variação de $A$ como sendo desprezível frente às variações da fase. Como $\phi$ é necessariamente uma função real (estamos descartando a hipóteses de dissipações), da relação acima podemos tirar que

$$
g^{\mu \nu} \partial_{\mu} \phi \partial_{\nu} \phi=0
$$

Desejamos que em cada ponto do espaço a onda seja descrita por um vetor de onda $\mathbf{k}$ e uma freqüência $\omega$. Vamos definir estas quantidades como

$$
\begin{gathered}
\mathbf{k}=\nabla \phi, \\
\omega=-\partial_{t} \phi .
\end{gathered}
$$

Vamos definir também o seguinte 4-vetor:

$$
k_{\mu}=(-\omega ; \mathbf{k})=\partial_{\mu} \phi
$$

Lembrando que a derivada covariante atua sobre uma função escalar da mesma forma que a derivada usual, podemos reescrever (3.16) como:

$$
\nabla_{\mu} \phi \nabla^{\mu} \phi=k^{\mu} k_{\mu}=0
$$

e tomando a derivada covariante da expressão anterior vem:

$$
k^{\sigma} \nabla_{\sigma} k^{\mu}=0
$$

Pelas duas ultimas equações concluímos que os raios de som são geodésicas tipo luz do espaço-tempo acústico.

\subsection{Espaços-tempos acústicos: algumas definições}

\subsubsection{Espaço-tempo acústico estacionário}

Em RG, um espaço-tempo é dito estacionário quando possui um campo de Killing tipo tempo. A existência deste campo implica que observadores seguindo suas linhas integrais não irão perceber variações no campo gravitacional. 
Vejamos qual é a condição para que a 4-velocidade dos observadores do laboratório seja um campo de Killing tipo tempo para a métrica acústica. Vamos cobrir o espaçotempo acústico com o sistema de coordenadas do laboratório. Tomando a derivada de Lie da métrica acústica com respeito ao campo $\left(\partial_{t}\right)^{a}$ temos

$$
£_{\partial_{t}} g_{\mu \nu}=\partial_{t} g_{\mu \nu}=0
$$

se $\left(\partial_{t}\right)^{a}$ é um campo de Killing. Por outro lado, o módulo quadrado do vetor $\left(\partial_{t}\right)^{a}$ com respeito à métrica acústica é

$$
g_{\mu \nu}\left(\partial_{t}\right)^{\mu}\left(\partial_{t}\right)^{\nu}=-\frac{\rho}{c}\left(c^{2}-\mathrm{v}^{2}\right)
$$

Logo, para que $\left(\partial_{t}\right)^{a}$ seja um campo de Killing tipo tempo é necessário satisfazer duas condições. A primeira condição é a de que as componentes da métrica no sistema de coordenadas do laboratório não dependam de $t$. Isto é satisfeito se o fluxo e a densidade do fluido forem independentes do tempo. A segunda condição é a de que a norma de $\left(\partial_{t}\right)^{a}$ seja negativa, o que só é possível se o fluxo for subsônico. Então, um espaço-tempo acústico é estacionário no sentido da RG quando é satisfeita a condição de estacionaridade hidrodinâmica e o fluxo é subsônico.

\subsubsection{Espaço-tempo acústico estático}

Um espaço-tempo estático é um caso particular de espaços-tempos estacionários. Em RG, um espaço-tempo é estático quando possui um campo de Killing tipo tempo $\xi^{a}$ e um conjunto de hiperfícies tipo espaço $\left\{\Sigma_{t}\right\}$ às quais $\xi^{a}$ é normal. Neste caso é sempre possível escrever o elemento de arco da seguinte forma

$$
d s^{2}=\left(\xi_{\mu} \xi^{\mu}\right)^{-1} d t^{2}+g_{i j} d x^{i} d x^{j}
$$

onde $g_{i j}$ é a métrica da seç̧ão espacial e $t$ é tal que $\xi^{a}=\nabla^{a} t$.

Novamente, vamos cobrir o espaço-tempo acústico com as coordenadas do laboratório. Desejamos saber quais são as condições necessárias para que $\left(\partial_{t}\right)^{a}$ seja campo de Killing e a métrica acústica tenha a forma acima. Se independente de $t$, o elemento de linha associado à métrica acústica é

$$
d s^{2}=\frac{\rho(\mathbf{x})}{c(\mathbf{x})}\left\{-\left[c^{2}(\mathbf{x})-\mathrm{v}^{2}(\mathbf{x})\right] d t^{2}-2 \mathbf{v}(\mathbf{x}) \cdot d \mathbf{x} d t+d \mathbf{x} \cdot d \mathbf{x}\right\}
$$


Vamos mostrar que existe um sistema de coordenadas no qual $g_{0 i}=0$. Para tanto, basta definir a coordenada temporal $\tau$ satisfazendo

$$
d t=d \tau-\frac{\mathbf{v} \cdot d \mathbf{x}}{c^{2}-\mathrm{v}^{2}}
$$

Substituindo a forma de $d t$ na expressão do elemento de linha, obtemos

$$
d s^{2}=\frac{\rho(\mathbf{x})}{c(\mathbf{x})}\left\{-\left[c^{2}(\mathbf{x})-\mathrm{v}^{2}(\mathbf{x})\right] d \tau^{2}+d \mathbf{x} \cdot d \mathbf{x}+\frac{[\mathbf{v}(\mathbf{x}) \cdot d \mathbf{x}]^{2}}{c^{2}(\mathbf{x})-\mathrm{v}^{2}(\mathbf{x})}\right\} .
$$

Desta forma do elemento de arco, concluímos, a rigor, que $\left(\partial_{\tau}\right)^{a}$ é o campo de Killing. Entretanto, sabemos que $\left(\partial_{\tau}\right)^{a}=\partial_{\tau} \mathbf{x}\left(\partial_{\mathbf{x}}\right)^{a}+\partial_{\tau} t\left(\partial_{t}\right)^{a}=\left(\partial_{t}\right)^{a}$. Para que esta transformação de coordenadas exista é suficiente que o vetor $\mathbf{v} /\left(c^{2}-\mathrm{v}^{2}\right)$ seja integrável. Isto é satisfeito se este vetor puder ser escrito como o gradiente de um campo escalar, ou de outra forma, se

$$
\nabla \times\left(\frac{\mathbf{v}}{c^{2}-\mathrm{v}^{2}}\right)=\mathbf{0} .
$$

Utilizando a hipótese de que o fluxo é irrotacional, a condição acima implica em

$$
\mathbf{v} \times \nabla\left(c^{2}-\mathrm{v}^{2}\right)=\mathbf{0}
$$

Por esta condição, um espaço-tempo acústico estacionário é estático se o fluxo é normal, com respeito à métrica do laboratório, às superfícies $c^{2}-\mathrm{v}^{2} \equiv$ cte.

\subsubsection{Ergoregião}

A norma de um vetor tangente à linha de mundo de um observador com respeito à métrica acústica é

$$
g_{\mu \nu} v^{\mu} v^{\nu} \propto-\left(c^{2}-\mathrm{v}^{2}\right) v^{0} v^{0}+\boldsymbol{v} \cdot \boldsymbol{v}-2 v^{0} \mathbf{v} \cdot \boldsymbol{v}<0 .
$$

Na região em que $\mathrm{v}>c$ temos que as duas primeiras parcelas são positivas definidas. Logo, para que a sentença acima valha, é necessário que $v^{0} \mathbf{v} \cdot \boldsymbol{v}>0$. Se usarmos $t$ como o parâmetro da curva integral de $v^{\mu}$, temos que $v^{0}=1$ e assim $\mathbf{v} \cdot \boldsymbol{v}>0$. Isto implica que na região de fluxo supersônico $\boldsymbol{v} \neq \mathbf{0}$. Conseqüentemente, o observador não pode ficar parado e é arrastado pelo fluido.

Vamos chamar de ergoregião a região na qual o observador é inevitavelmente arrastado pelo fluido. Pelo fato de o fluxo ser supersônico, o módulo quadrado do vetor que 
escolhemos como dando a direção temporal será

$$
\left(\partial_{t}\right)^{a}\left(\partial_{t}\right)_{a}=g_{t t}=-\frac{\rho}{c}\left(c^{2}-\mathrm{v}^{2}\right)>0 .
$$

Logo, este vetor passa a ser tipo espaço na ergoregião. Vamos definir a ergoregião como aquela em que o vetor $\left(\partial_{t}\right)^{a}$ passa a ser tipo espaço. Esta definição é livre de ambigüidades, pois $t$ é uma coordenada temporal "natural" por ser o tempo físico do laboratório. Em outras palavras, o laboratório fornece uma noção de "repouso absoluto".

\subsubsection{Horizonte de eventos}

Considere uma superfície fechada no espaço. Se sobre esta superfície, em todos os pontos, o fluxo aponta para dentro e sua componente normal, com respeito à métrica do laboratório, a ela é maior que a velocidade local do som, então não há meios de o som escapar de seu interior. A estas superfícies dá-se o nome de superfícies de aprisionamento. Um conjunto destas superfícies dá origem à região conhecida como região de aprisionamento. A borda desta região definiremos como o horizonte de eventos aparente.

O horizonte de eventos absoluto é definido como a borda da região do espaço-tempo acústico da qual geodésicas tipo luz não podem escapar. Este é o horizonte futuro de eventos. O horizonte passado de eventos é a borda da região a qual nenhuma geodésica tipo luz dirigida ao futuro pode atingir. Estas definições dependem, é claro, das definições de infinito nulo passado e infinito nulo futuro.

Em geometrias estacionárias, o horizonte de eventos aparente e o horizonte de eventos absoluto coincidem. Definamos, então, o horizonte de eventos $\mathcal{H}$ como a hiperfície pertencente a um espaço-tempo acústico gerado por um fluxo convergente sobre a qual $c^{2}-\mathrm{v}_{\perp}^{2}=0$. $\mathrm{O}$ vetor $\mathbf{v}_{\perp}$ é o vetor normal, em relação à métrica do laboratório, às superfícies geradas pelas intersecção entre $\mathcal{H}$ e as hiperfícies $t$ constante. Esta definição implica que o horizonte de eventos está sempre contido na ergoregião. O espaço-tempo acústico que apresenta um horizonte de eventos é chamado de buraco sônico ou de buraco negro acústico ou ainda de buraco mudo. 


\subsubsection{Gravidade superficial}

\section{Espaços-tempos acústicos estáticos}

Em espaços-tempos acústicos estáticos, pelo fato de o fluxo ser normal, em relação à métrica do laboratório, às superfícies $c^{2}-\mathrm{v}^{2} \equiv$ cte, temos que $\mathbf{v} \propto \nabla\left(c^{2}-\mathrm{v}^{2}\right)$. Isto implica que o 4-vetor normal, em relação à métrica acústica, às hiperfícies $c^{2}-\mathrm{v}^{2} \equiv$ cte é proporcional à 4-velocidade do fluido. A 4-velocidade do fluido, por sua vez, é proporcional ao campo de Killing tipo tempo. Isto pode ser visto pela relação (3.16). Logo, o campo de Killing $\left(\partial_{t}\right)^{a}$ é normal, com respeito à métrica acústica, ao horizonte de eventos em espaços-tempos acústicos estáticos.

A quantidade $\left(\partial_{t}\right)^{a}\left(\partial_{t}\right)_{a}$, quando calculada sobre o horizonte, nos volta

$$
\left.\left(\partial_{t}\right)^{a}\left(\partial_{t}\right)_{a}\right|_{\mathcal{H}}=0
$$

para qualquer ponto pertencente ao horizonte. Logo, o vetor $\left.\nabla^{b}\left[\left(\partial_{t}\right)^{a}\left(\partial_{t}\right)_{a}\right]\right|_{\mathcal{H}}$ é normal, em relação à métrica acústica, a $\mathcal{H}$. Vamos definir a gravidade superficial $\kappa$ neste caso como o coeficiente de proporcionalidade entre este vetor e $\left(\partial_{t}\right)^{a}$ da seguinte forma:

$$
\left.\nabla^{b}\left[\left(\partial_{t}\right)^{a}\left(\partial_{t}\right)_{a}\right]\right|_{\mathcal{H}}=-\frac{2}{c} \kappa\left(\partial_{t}\right)^{b}
$$

É justificado extrair o fator $1 / c$ na expressão acima, pois desejamos que $\kappa$ tenha unidade de aceleração. Calculando a derivada vem

$$
\left.\nabla^{b}\left[\left(\partial_{t}\right)^{a}\left(\partial_{t}\right)_{a}\right]\right|_{\mathcal{H}}=\left.\frac{1}{c} \hat{\mathbf{n}} \cdot \nabla\left(c^{2}-\mathrm{v}^{2}\right)\right|_{\mathrm{v}=c}\left(\partial_{t}\right)^{a}
$$

de onde tiramos a seguinte forma para a gravidade superficial:

$$
\kappa=-\left.\frac{1}{2} \hat{\mathbf{n}} \cdot \nabla\left(c^{2}-\mathrm{v}^{2}\right)\right|_{\mathrm{v}=c} .
$$

Aqui $\hat{\mathbf{n}}$ denota o vetor unitário normal, com respeito à métrica do laboratório, às superfícies $c^{2}-\mathrm{v}^{2}=0$.

\section{Espaços-tempos acústicos estacionários}

Em espaços-tempos estacionários geralmente $\mathbf{v} \times \nabla\left(c^{2}-\mathrm{v}^{2}\right) \neq 0$. Logo, não podemos afirmar que $\left(\partial_{t}\right)^{a}$ é normal, em relação à (3.12), a $\mathcal{H}$. Isto gera dificuldades técnicas para definirmos a gravidade superficial. 
Para contorná-las, temos que construir um campo vetorial que gere todo o horizonte. Vejamos como. Para seguir, vamos assumir que de alguma forma conhecemos a superfície $c^{2}-\mathrm{v}_{\perp}^{2}=0$ e que ao menos em sua vizinhança somos capazes de decompor o fluxo do fluido em duas parcelas, $\mathbf{v}=\mathbf{v}_{\perp}+\mathbf{v}_{\|}$, tais que

$$
\mathbf{v}_{\perp} \times \nabla\left(c^{2}-\mathrm{v}_{\perp}^{2}\right)=\mathbf{0}, \quad \mathbf{v}_{\|} \cdot \nabla\left(c^{2}-\mathrm{v}_{\perp}^{2}\right)=0 .
$$

Pela definição, a cada instante $t$ o vetor $\mathbf{v}_{\perp}$ é normal e $\mathbf{v}_{\|}$é tangente às superfícies $c^{2}-\mathrm{v}_{\perp}^{2} \equiv$ cte, com respeito à métrica do laboratório. Considere agora o seguinte campo vetorial:

$$
L^{\mu}=\left(1 ; \mathbf{v}_{\|}\right)
$$

Por construção, este campo gera, a todo instante, as hiperfícies $c^{2}-\mathrm{v}^{2} \equiv$ cte e, em particular, o horizonte de eventos. O campo $L^{a}$ não é um campo de Killing, entretanto calculando a sua norma,

$$
L^{a} L_{a}=-\frac{\rho}{c}\left(c^{2}-\mathrm{v}_{\perp}^{2}\right),
$$

obtemos que $L^{a}$ é tipo luz sobre $\mathcal{H}$. Tomando a derivada covariante da norma de $L^{a}$ e utilizando a definição de gravidade superficial, vem:

$$
\kappa=-\left.\frac{1}{2} \hat{\mathbf{n}} \cdot \nabla\left(c^{2}-\mathrm{v}_{\perp}^{2}\right)\right|_{\mathrm{v}_{\perp}=c} \cdot
$$

Este resultado para a gravidade superficial em análogos difere daquele usado em [14], pois, em seu modelo, Unruh assumiu que a velocidade do som no meio era uniforme.

\subsection{Adicionando viscosidade}

Como definimos no início deste capítulo, no fluido ideal o transporte de momentum e energia dá-se pelo fato de tirarmos partículas de um lugar e colocá-las em outro e pela pressão interna. Em fluidos reais, além destes efeitos, temos que o movimento relativo entre suas partes implica na transferência irreversível de energia e momentum. Isto é o que chamamos de viscosidade. A viscosidade está ligada a fricções internas e outros processos dissipativos. Logo, é um fenômeno irreversível.

Um elemento de fluido, ao deslizar sobre outro, exerce uma força no sentido de seu movimento sobre o elemento de baixo. A força viscosa aparece devido ao deslizamento 
destas camadas, isto é, depende das diferenças de velocidades entre as partes do fluido. Logo, esta força dissipativa deve estar relacionada a derivadas espaciais do fluxo. De fato, a experiência nos diz que, para gradientes do fluxo pequenos, a força viscosa deve ser proporcional a gradientes de $\mathbf{v}$.

Considerações deste tipo nos levam a seguinte generalização da equação de Euler:

$$
\rho\left[\partial_{t} \mathbf{v}+(\mathbf{v} \cdot \nabla) \mathbf{v}\right]=-\nabla P+\eta \nabla^{2} \mathbf{v}+\left(\zeta+\frac{1}{3} \eta\right) \nabla(\nabla \cdot \mathbf{v}) .
$$

A equação da continuidade (3.2) permanece inalterada. A (3.17) é a famosa equação de Navier-Stokes. Aqui $\eta$ e $\zeta$ são parâmetros fenomenológicos conhecidas por viscosidade e segunda viscosidade, nesta ordem, que podem depender da temperatura. Estes parâmetros aparecem quando construímos o tensor de stress mais geral devido à viscosidade impondo que ele seja simétrico e supondo que dependa somente de gradientes do fluxo. A partir dos gradientes de $\mathbf{v}$ há somente dois tensores simétricos que podem ser construídos: o diádico $\nabla \mathbf{v}$ simetrizado e o divergente de $\mathbf{v}$ multiplicando uma delta de Kronecker. Os parâmetros $\eta$ e $\zeta$ são usados para fazer uma linear arbitrária destes dois tensores. Para mais detalhes ver [37]. Vejamos quais as conseqüências da viscosidade na derivação da equação para o som, como delineada na seção 3.1 .

Vamos supor novamente que o fluxo é irrotacional. O processo de linearização da equação (3.17) implica no seguinte termo adicional no lado esquerdo da primeira equação de (3.8):

$$
\frac{1}{c^{2}}\left(\frac{4}{3} \eta+\zeta\right) \nabla^{2} \psi_{1}
$$

A equação para as perturbações sobre o potencial do fluxo fica

$$
\nabla^{\mu} \nabla_{\mu} \psi_{1}=-\left(\frac{4}{3} \eta+\zeta\right) \frac{c}{\rho_{0}}\left(\partial_{t}+\mathbf{v} \cdot \nabla\right)\left(\frac{1}{\rho_{0} c^{2}} \nabla^{2} \psi_{1}\right)
$$

onde $\nabla_{\mu}$ é a derivada covariante relacionada à métrica (3.12). O lado direito da expressão acima pode ser escrito em termos da 4 -velocidade $u^{\mu}$ do fluido,

$$
\partial_{t}+\mathbf{v} \cdot \nabla=\sqrt{\rho_{0} c} \mathrm{u}^{\mu} \partial_{\mu}
$$

então,

$$
\nabla^{\mu} \nabla_{\mu} \psi_{1}=-\left(\frac{4}{3} \eta+\zeta\right) \sqrt{\frac{c^{3}}{\rho_{0}}} \mathrm{u}^{\mu} \nabla_{\mu}\left(\frac{1}{\rho_{0} c^{2}} \nabla^{2} \psi_{1}\right)
$$


Com $\eta$ e $\zeta$ não nulos, a equação (3.18) claramente não é mais covariante sob transformações gerais de coordenadas. Isto é um problema se desejamos simular algumas das conseqüências da RG sobre campos. Entretanto, esperamos que análogos também sejam capazes de nos guiar além da RG. Com este propósito, talvez efeitos tipo viscosidade sejam interessantes. Pela forma da equação (3.18), podemos prever que a relação de dispersão dos modos não será do tipo freqüência proporcional ao módulo do vetor de onda. Logo, a relação entre momentum e energia das partículas associadas não será mais aquela da RR. $\mathrm{Na}$ literatura diz-se, então, que estes efeitos quebram a invariância de Lorentz.

A viscosidade está intimamente ligada à estrutura microscópica da matéria que compõe o fluido. Ela só se torna importante quando temos intensos gradientes de velocidade, o que implica que para os modos o fluido é tão mais viscoso quanto menor seu comprimento de onda. Via de regra, a viscosidade é relevante para modos cujo comprimento de onda é da ordem do livre caminho médio dos átomos. Neste regime, os modos passam a ser sensíveis a estrutura íntima do fluxo sobre o qual definimos o espaço-tempo acústico.

Isto nos permite afirmar que a estrutura microscópica do fluido, em certas escalas de tempo e tamanho, podem levar à quebra da invariância de Lorentz. Esta situação é análoga a que se espera para modos testando o espaço-tempo em tempos e tamanhos que estão abaixo da escala de Planck.

\subsection{Alguns apontamentos experimentais}

O principal interesse em modelos de matéria condensada análogos a sistemas gravitacionais reside no vislumbre da possibilidade de testarmos o efeito Hawking em laboratório. Como argumentamos na seção 2.2, a radiação Hawking deve-se ao fato de que a formação de um horizonte de eventos perturba o vácuo quântico. Para que seja possível implementar processos deste tipo em laboratório é necessário produzir fluxos supersônicos muito frios. Isto implica em algumas dificuldades experimentais.

É natural que um espaço-tempo acústico com um horizonte de eventos seja construído através de um escoamento que em algum momento torna-se supersônico. Implementar fluxos supersônicos em laboratório e mantê-los estáveis não parece ser tarefa simples. A principal dificuldade decorre do fato de que escoamentos supersônicos podem eventual- 
mente gerar ondas de choque no fluido. Uma onda de choque consiste em uma superfície de descontinuidade que se propaga pelo fluido; sobre esta superfície quantidades como v, $\rho$ e $P$ tornam-se descontínuas. Quando uma onda de choque forma-se, ela perturba o fluido a um ponto tal que não podemos mais tomar o fluxo como isentrópico. Grosseiramente falando, podemos entender a formação das ondas de choque da seguinte forma. Considere frentes de ondas de som que se propagam pelo fluido. Quando o escoamento é supersônico, estas frentes de onda são dragadas pelo fluxo e se "empilham" umas sobre as outras. Isto é análogo ao que ocorre quando alguma embarcação singra no mar a uma velocidade superior à velocidade do som na superfície da água. Ao cortar a superfície, a quilha da embarcação cria perturbação que não conseguem escapar para longe e se empilham e quebram na proa. Ao se empilharem, as ondas de som no fluido criam uma zona de alta pressão que acaba por implicar, devido à não-linearidade da hidrodinâmica, em superfícies de descontinuidade.

Numa possível realização experimental de um modelo análogo , estaríamos interessados em verificar de que forma as inomogeneidades do meio afetam suas flutuações de ponto zero. À temperatura ambiente, estas flutuações intrinsecamente quânticas são mascaradas pelo balançar térmico das partículas do meio. A temperatura necessária para que efeitos do tipo radiação Hawking sejam perceptíveis é tão mais baixa quanto menor o gradiente do fluxo, no caso do modelo do fluido. Em [14], Unruh estima em $10^{-7} \mathrm{~K}$ a temperatura da radiação Hawking produzida por um fluxo supersônico que varia 300 m/s por milímetro. Isto é, uma variação do fluxo igual a velocidade do som no ar numa região com o comprimento da menor divisão de uma régua escolar para produzir um décimo de bilionésimo de Kelvin! Via de regra, a velocidade do som no meio é inversamente proporcional à densidade de matéria, o que torna ainda mais difícil buracos mudos em um copo d'água.

Fica claro, então, que é imperativo que a temperatura do meio seja frações de Kelvin para que tenhamos alguma esperança de medir radiação Hawking em laboratório. A estas temperaturas, quase todos os fluidos que conhecemos à temperatura ambiente tornam-se sólidos. Além disso, a temperatura é tão baixa que o comprimento de onda térmico passa a ser comparável com o livre caminho médio das partículas do fluido. Nestas situações, 
temos que levar em conta a natureza quântica dos próprios constituintes do fluxo; estes são os chamados fluidos quânticos. De fato, próximo ao zero absoluto, somente o hélio apresenta uma fase fluida. São conhecidos dois isótopos estáveis do hélio, o $\mathrm{He}^{3}$ e o $\mathrm{He}^{4}$, que têm propriedades absolutamente distintas. $\mathrm{O} \mathrm{He}^{4}$, em particular, apresenta algumas propriedades interessantes abaixo de $2,19 \mathrm{~K}$; a mais notável delas é a de apresentar uma fase superfluida descoberta em 1938 por P. L. Kapitza. O He II, como é conhecida esta fase do hélio, apresenta uma mistura de fluxos, sendo que um deles é idêntico ao de um fluido ideal cujo escoamento é irrotacional. Este é um sistema bastante promissor, do ponto de vista experimental, para se implementar modelos análogos.

Apesar das dificuldades, modelos análogos são, sem sombra de dúvidas, o atalho mais curto para se verificar algumas das predições da TQCEC. Os desafios tecnológicos são muito menores do que os envolvidos em outros meios. O próprio Unruh afirma, em [14], ao comentar o pequeno valor para a temperatura da radiação de fônons em análogos, o que segue. "This is a rather low temperature, and it is probably undetectable in the presence of turbulent instabilities, etc., which would arise in trying to drive the fluid transsonically through a small nozzle. It is, however, a much simpler experimental task than creating a $10^{-18}$ cm black hole." 


\section{Capítulo 4}

\section{Aplicações do modelo do fluido}

Neste capítulo utilizaremos o modelo do fluido para construir geometrias acústicas que simulem horizontes de eventos e mostrar algumas conseqüências da TQCEC neste contexto. Na seção 4.1 mostraremos como podemos utilizar as equações da hidrodinâmica para construir os buracos mudos em uma, duas e três dimensões. Na seção 4.2 utilizaremos uma montagem tipo bocal de Laval para derivarmos o resultado de Hawking via o espaço de momenta. Esta análise será útil ao introduzirmos o problema dos modos trans-planckianos. Nas seções 4.3 e 4.4 trataremos, respectivamente, dos problemas do back-reaction e dos modos trans-planckianos no contexto de análogos. Na seção 4.3 mostraremos um formalismo geral desenvolvido em [34] para tratar o back-reaction em análogos e qual sua conseqüência quando aplicado ao escoamento de um fluido em um bocal de Laval. Na seção 4.4 mostraremos uma forma de tratar os modos trans-planckianos em análogos como proposta por Jacobson em [17].

\subsection{Buracos mudos}

\subsubsection{Fluxos unidimensionais}

Suponha um escoamento de um fluido ideal que dependa da coordenada $z$ e seja homogêneo no plano $x y$. Isto pode ser obtido numa configuração em que o fluxo do fluido ao longo de z é muito maior que nas outras direções. Considere o caso estacionário, onde todas as quantidades que descrevem o fluido são independentes de $t$. Na ausência de 
fontes e forças externas, as equações da continuidade e de Euler, nesta ordem, nos dão

$$
\begin{aligned}
& \nabla \cdot\left[\rho(z) \mathrm{v}_{z}(z) \hat{\mathbf{z}}\right]=0 \\
& \mathrm{v}_{z} \frac{d \mathrm{v}_{z}}{d z}+\frac{c^{2}}{\rho} \frac{d \rho}{d z}=0 .
\end{aligned}
$$

Vamos supor que o escoamento está restrito a um tubo cuja secção transversal tem área $A(z)$. Integrando a equação da continuidade num certo volume do tubo, levando em consideração que o fluxo se anula sobre as paredes, temos que $\rho(z) A(z) \mathrm{v}_{z} \equiv$ cte. Usando esta relação para eliminar $\rho$ na equação de Euler, vem

$$
\frac{\frac{d \mathrm{v}_{z}}{d z}}{\mathrm{v}_{z}}\left(\frac{\mathrm{v}_{z}^{2}-c^{2}}{c^{2}}\right)=\frac{\frac{d A}{d z}}{A}
$$

Vejamos as conseqüências deste resultado. Considere que o fluxo é subsônico. Se a área da secção transversal do tubo decresce, então $\frac{d A}{d z}<0$ e a velocidade cresce, pois $\mathrm{v}_{z}^{2}-c^{2}<0$ e $\frac{d \mathrm{v} z}{d z} \propto-\frac{d A}{d z}$. Considere agora que o fluxo é supersônico. Neste caso $\mathrm{v}_{z}^{2}-c^{2}>0$, o que implica que $A$ e $\mathrm{v}_{z}$ crescem ou decrescem juntos. Podemos usar estas relações entre a área de secção transversal e o fluxo para construir um tubo que somente por conta de seu perfil acelere o fluxo até a velocidade do som. Para tanto, basta que $A$ decresça até que a velocidade do fluido seja igual a velocidade local do som. De (4.1) vemos que quando $\mathrm{v}_{z}=c, \frac{d A}{d z}=0$. Logo, a velocidade do som é atingida pelo meio no ponto de mínimo da área de secção transversal. O perfil que produz este tipo de escoamento é conhecido como bocal de Laval.

Com um bocal de Laval somos capazes de produzir um horizonte de eventos sônico $\mathcal{H}$ no ponto $z=z_{\mathcal{H}}$ onde $A(z)$ é mínima. A gravidade superficial sobre o horizonte pode ser escrita em termos da função $A(z)$,

$$
\kappa=-\left.c \frac{d}{d z}\left(c-\mathrm{v}_{z}\right)\right|_{\mathcal{H}}=-\frac{1}{4}\left[\frac{d c^{2}}{d z}-\sqrt{\left(\frac{d c^{2}}{d z}\right)^{2}+\frac{8 c^{4}}{A} \frac{d^{2} A}{d z^{2}}}\right]_{z=z_{\mathcal{H}}} .
$$

No caso em que a velocidade do som no meio é constante, a expressão anterior reduz-se a

$$
\kappa=\left.\sqrt{\frac{c^{4}}{2 A} \frac{d^{2} A}{d z^{2}}}\right|_{z=z_{\mathcal{H}}} .
$$

Se o fluido é submetido a uma densidade de força por unidade de volume $\mathbf{f}=\mathrm{f}(z) \hat{\mathbf{z}}$, a relação (4.1) fica

$$
\frac{\frac{d \mathrm{v}_{z}}{d z}}{\mathrm{v}_{z}}\left(\frac{\mathrm{v}_{z}^{2}-c^{2}}{c^{2}}\right)-\frac{\mathrm{f}}{\rho c^{2}}=\frac{\frac{d A}{d z}}{A} .
$$


Isto implica que

$$
\left.\frac{\frac{d A}{d z}}{A}\right|_{z=z_{\mathcal{H}}}=-\left.\frac{\mathrm{f}}{\rho c^{2}}\right|_{z=z_{\mathcal{H}}} .
$$

Logo, o horizonte de eventos sônico não se localiza mais no ponto de mínimo da área de secção transversal. A gravidade superficial fica

$\kappa=-\frac{1}{4}\left\{\frac{d c^{2}}{d z}-\sqrt{\left(\frac{d c^{2}}{d z}\right)^{2}+8 c^{4}\left[\frac{1}{A} \frac{d^{2} A}{d z^{2}}-\frac{1}{A^{2}}\left(\frac{d A}{d z}\right)^{2}+\frac{1}{\rho c^{2}} \frac{d \mathrm{f}}{d z}-\frac{\mathrm{f}}{\left(\rho c^{2}\right)^{2}} \frac{d}{d z}\left(\rho c^{2}\right)\right]}\right\}_{z=z_{\mathcal{H}}}$.

Uma força externa é mais um artificio que pode ser usado para regularmos a posição do horizonte e a gravidade superficial.

\subsubsection{Fluxos bidimensionais}

Suponha um fluxo laminar no plano $x y$. Vamos considerar que o escoamento é muito mais intenso no plano $x y$ do que na direção transversal, de forma que seja possível aproximá-lo por um fluxo puramente bidimensional. Impondo simetria radial ao fluxo, decorrem das equações da continuidade e de Euler, nesta ordem, que

$$
\begin{gathered}
\partial_{t} \rho+\frac{1}{r} \partial_{r}\left(r \rho \mathrm{v}_{r}\right)=g, \\
\rho \partial_{t} \mathbf{v}+\rho \mathrm{v}_{r} \partial_{r} \mathbf{v}-\hat{\mathbf{r}} \partial_{r} P=\mathbf{f},
\end{gathered}
$$

onde $g$ e f são, respectivamente, uma fonte ou sorvedouro e um campo de força por unidade de volume. Se o fluxo é estacionário e o fluido incompressível, a equação da continuidade nos dá

$$
\mathrm{v}_{r}=\frac{A}{r}
$$

na presença de uma fonte ou sorvedouro tipo delta. Da condição de vorticidade nula vem

$$
\mathrm{v}_{\varphi}=\frac{B}{r}
$$

Aqui, $A$ e $B$ são constantes. Logo, temos o seguinte campo de velocidade para o fluxo de fundo:

$$
\mathbf{v}=\frac{A}{r} \hat{\mathbf{r}}+\frac{B}{r} \hat{\varphi}
$$

Este campo tem as seguintes linhas integrais:

$$
r=r_{0}+\frac{A}{B}\left(\varphi-\varphi_{0}\right)
$$


Logo, os elementos de fluido seguem espirais de Arquimedes.

Se $A$ e $B$ são diferentes de zero, então temos um vórtice em torno de uma fonte, se $A>0$, ou em torno de um sorvedouro, se $A<0$. A métrica acústica associada a este escoamento é

$$
g_{\mu \nu}=\frac{\rho}{c}\left[\begin{array}{ccc}
-\left(c^{2}-\frac{A^{2}+B^{2}}{r^{2}}\right) & -\frac{A}{r} & -B \\
-\frac{A}{r} & 1 & 0 \\
-B & 0 & r^{2}
\end{array}\right] .
$$

Um fluxo tal que $A<0(A>0)$ é capaz de simular um buraco negro (buraco branco) bidimensional com rotação, se $B \neq 0$.

A ergoregião é o lugar geométrico dos pontos tais que $\mathbf{v}^{2}=c^{2}$. Logo, a coordenada radial destes pontos é

$$
r_{\text {ergoregião }}=\frac{\sqrt{A^{2}+B^{2}}}{c} .
$$

A coordenada radial dos pontos do horizonte de eventos satisfazem $\mathbf{v}_{\perp}^{2}\left(r_{\mathcal{H}}\right)=c^{2}$. Neste caso, como o fluxo tem simetria radial, $\mathbf{v}_{\perp}=\mathbf{v} \cdot \hat{\mathbf{r}} \hat{\mathbf{r}}$. Então

$$
r_{\mathcal{H}}=\frac{|A|}{c}
$$

Logo, em um buraco mudo sem rotação, a fronteira da ergoregião coincide com o horizonte de eventos. A gravidade superficial do buraco mudo será

$$
\kappa=\left\{\begin{array}{r}
-\frac{c^{3}}{|A|}, \\
\frac{c^{3}}{|A|} .
\end{array}\right.
$$

O sinal negativo corresponde a um buraco branco e o sinal positivo corresponde a um buraco negro.

\subsubsection{Fluxos tridimensionais}

\section{Fluxo convergente}

Considere um fluxo tridimensional esfericamente simétrico. Neste caso, as equações da continuidade e de Euler são

$$
\begin{gathered}
\partial_{t} \rho+\frac{1}{r^{2}} \partial_{r}\left(r^{2} \rho \mathrm{v}_{r}\right)+\frac{1}{r \tan \theta} \rho \mathrm{v}_{\theta}=g \\
\partial_{t} \mathbf{v}+\mathrm{v}_{r} \partial_{r} \mathbf{v}-\hat{\mathbf{r}} \partial_{r} P=\mathbf{f} .
\end{gathered}
$$


A condição de fluxo irrotacional implica que

$$
\mathrm{v}_{\varphi}=0, \quad \mathrm{v}_{\theta}=\frac{B}{r}
$$

Se o fluxo é independente do tempo, da equação da continuidade decorre que $B=0$, já que todas as outras parcelas são funções somente de $r$. Por fim, ficamos com o seguinte sistema de equações para resolver:

$$
\begin{gathered}
\frac{1}{r^{2}} \frac{d}{d r}\left(r^{2} \rho \mathbf{v}_{r}\right)=g, \\
\rho \mathrm{v}_{r} \frac{d \mathrm{v}_{r}}{d r}-\frac{d P}{d r}=\mathrm{f}_{r} .
\end{gathered}
$$

Suponha que na ausência de forças o fluido é sugado de forma esfericamente simétrica para um único ponto. Neste caso, podemos escrever o sorvedouro como $g(r)=\frac{A}{r^{2}} \delta(r)$. Aqui, $A$ é uma constante que dá a intensidade com que o fluido é sugado. Se impusermos que infinitamente distante do sorvedouro o fluido está parado, temos que a equação da continuidade nos dá

$$
\rho \mathrm{v}_{r}=\frac{A}{r^{2}} .
$$

Voltando o resultado anterior na equação de Euler, vem a relação entre a densidade e a pressão,

$$
\frac{d P}{d r}=\left(\frac{A}{r}\right)^{2} \frac{d}{d r}\left(\frac{1}{\rho r^{2}}\right) .
$$

Esta equação pode ser resolvida se conhecida a equação de estado $P=P(\rho)$.

\section{Buraco mudo conforme à métrica de Schwarzschild}

Desejamos encontrar quais condições sobre o fluxo são necessárias para que o elemento de linha do espaço-tempo acústico (3.13) tenha a mesma forma do elemento de linha do espaço-tempo de Schwarzschild (2.45). Para tanto, basta encontrarmos um sistema de coordenadas em que a secção espacial de Schwarzschild seja plana. Este novo sistema de coordenadas está relacionado às coordenadas de Schwarzschild por

$$
d t^{2}=d T^{2}+\frac{2 M / r}{(1-2 M / r)^{2}} d r^{2}-\frac{2 \sqrt{2 M / r}}{1-2 M / r} d T d r
$$

Substituindo a relação anterior em (2.45), vem o seguinte elemento de linha:

$$
d s^{2}=-\left(1-\frac{2 M}{r}\right) d T^{2}+2 \sqrt{\frac{2 M}{r}} d T d r+d r^{2}+r^{2}\left(d \theta^{2}+\operatorname{sen}^{2} \theta d \varphi^{2}\right) .
$$


Este novo sistema de coordenadas é conhecido por sistema de Painlevé-Gullstrand.

Para que o elemento de linha (3.13) tenha a forma de Painlevé-Gullstrand, é necessário que a velocidade do som no meio seja constante e que o fluxo seja estacionário, convergente, esfericamente simétrico e que a componente do fluxo na direção radial seja

$$
\mathrm{v}_{r}=-\sqrt{\frac{2 M}{r}} .
$$

Estas imposições sobre o fluxo implicam, através da equação da continuidade, que

$$
\rho \propto \frac{1}{r^{3 / 2}}
$$

Logo, através do modelo do fluido, somos somente capazes de simular métricas conformes à métrica de Schwarzschild. O fator conforme $\Omega^{2}$ é proporcional a $1 / r^{3 / 2}$, já que a métrica acústica (3.12) tem todas suas componentes multiplicadas pelo fator $\rho / c$. Isto não é um problema, ao menos para a reprodução do efeito Hawking em laboratório via análogos, já que a gravidade superficial é um invariante conforme.

\section{Buraco mudo canônico}

Um buraco mudo canônico é aquele espaço-tempo acústico gerado pelo fluxo estacionário e convergente, esfericamente simétrico, de um fluido cuja densidade é constante. Estas suposições, quando aplicadas à equação da continuidade, implicam que

$$
\mathbf{v}=-c\left(\frac{r_{\mathcal{H}}}{r}\right)^{2} \hat{\mathbf{r}}
$$

onde $r_{\mathcal{H}}$ é a coordenada radial dos pontos tais que $\mathrm{v}_{r}=c$.

A este fluxo está associado o seguinte intervalo invariante do espaço-tempo acústico:

$$
d s^{2}=\frac{\rho}{c}\left[-c^{2}\left(1-\frac{r_{\mathcal{H}}^{4}}{r^{4}}\right) d t^{2}+2 c\left(\frac{r_{\mathcal{H}}}{r}\right) d t d r+d r^{2}+r^{2}\left(d \theta^{2}+\operatorname{sen}^{2} \theta d \varphi^{2}\right)\right] .
$$

Podemos colocá-lo na forma do elemento de linha de um espaço-tempo estático. Isto é possível se fizermos a seguinte transformação de coordenadas:

$$
d t=d \tau+\frac{\left(r_{\mathcal{H}} / r\right)^{2}}{c\left[1-\left(r_{\mathcal{H}} / r\right)^{4}\right]} d r .
$$

Substituindo na forma anterior do elemento de linha do espaço-tempo acústico, temos

$$
d s^{2}=-c^{2}\left(1-\frac{r_{\mathcal{H}}^{4}}{r^{4}}\right) d \tau^{2}+\frac{1}{1-\left(\frac{r_{\mathcal{H}}}{r}\right)^{4}} d r^{2}+r^{2}\left(d \theta^{2}+\operatorname{sen}^{2} \theta d \varphi^{2}\right)
$$


Em [22], M. Visser afirma que seria possível produzir uma versão dependente do tempo de um buraco mudo canônico na parte externa de uma bolha cujo raio oscila suficientemente rápido para gerar um horizonte de eventos.

\subsection{Radiação Hawking no bocal de Laval}

Considere um fluido cujo escoamento se dá em uma montagem tipo bocal de Laval. Suponha que o fluxo evolui de forma tal que em algum momento forma-se um horizonte sônico na cintura do bocal. Suponha que o fluxo é quase unidimensional. Esta montagem dá origem ao seguinte espaço-tempo acústico:

$$
d s^{2}=\frac{\rho}{c}\left[-\left(c^{2}-\mathrm{v}_{z}^{2}\right) d t^{2}-2 \mathrm{v}_{z} d t d z+d x^{2}+d y^{2}+d z^{2}\right]
$$

onde estamos supondo que o escoamento dá-se ao longo da direção do eixo z. Sabemos que os modos responsáveis pela criação de partículas são aqueles que passam imediatamente próximos ao horizonte de eventos. Logo, é suficiente resolvermos a equação de campo muito próximo do horizonte. Não estamos interessados no fluxo de partículas transiente, o que nos permite considerar o fluxo já em seu regime independente do tempo. Isto nos permite aproximar o fluxo por

$$
\mathrm{v}_{z}(z) \simeq-c\left(z_{\mathcal{H}}\right)+\frac{\kappa}{c\left(z_{\mathcal{H}}\right)}\left(z-z_{\mathcal{H}}\right)
$$

onde estamos supondo que $\kappa\left(z-z_{\mathcal{H}}\right) / c\left(z_{\mathcal{H}}\right) \ll 1$. Nesta região, a equação da continuidade impõe que

$$
\rho(z) \simeq \rho\left(z_{\mathcal{H}}\right)\left[1+\frac{\kappa}{c\left(z_{\mathcal{H}}\right)}\left(z-z_{\mathcal{H}}\right)\right]
$$

Como muito próximo do horizonte a densidade varia muito pouco, vamos aproximá-la por $\rho\left(z_{\mathcal{H}}\right)$. Esta aproximação implicará em desprezarmos um termo tipo potencial que simplesmente diminui a intensidade do fluxo de partículas, sem alterar o seu espectro. Vamos supor que a equação de estado $P=P(\rho)$ é tal que tomar a velocidade do som no meio como homogênea e igual a $c\left(z_{\mathcal{H}}\right)$ é uma boa apriximação nas imediações do horizonte sônico.

A equação para os modos, nesta geometria, tem a forma

$$
-\partial_{t}^{2} \psi-\partial_{t}\left(\mathrm{v}_{z} \partial_{z} \psi\right)-\partial_{z}\left(\mathrm{v}_{z} \psi\right)+\partial_{z}\left[\left(c^{2}-\mathrm{v}_{z}^{2}\right) \partial_{z} \psi\right]=0
$$


Como a métrica é independente do tempo, é útil escrever os modos como

$$
\psi(z, t)=\int_{0}^{\infty} d \omega \psi_{\omega}(z) e^{-\mathrm{i} \omega t}
$$

Aqui integramos somente em $\omega$ positivo pois desejamos encontrar a forma dos modos do campo que sejam de freqüência positiva. Da transformação acima, temos a seguinte equação para $\psi_{\omega}$ :

$$
\left[\omega+\mathrm{i} \frac{d}{d z}\left(\mathrm{v}_{z}-c\right)\right]\left[\omega+\mathrm{i}\left(\mathrm{v}_{z}+c\right) \frac{d}{d z}\right] \psi_{\omega}=0
$$

onde estamos definondo que os operadores diferenciais atuam sobre qualquer função de $z$ que esteja à sua direita.

Vamos resolver o problema de uma forma diferente daquela que utilizamos na seção 2.2, utilizaremos o espaço de momenta dos modos out. Esta representação se mostrará útil quando tratarmos dos modos trans-planckianos em análogos. Vamos fazer a seguinte transformação:

$$
\tilde{\psi}_{\omega}(p)=\int_{-\infty}^{\infty} d z \frac{e^{-\mathrm{i} p z}}{\sqrt{2 \pi}} \psi_{\omega}(z)
$$

A equação para $\tilde{\psi}_{\omega}$ é obtida multiplicando a equação para $\psi_{\omega}$ por $\frac{e^{-i p z}}{\sqrt{2 \pi}}$ e integrando em $z$

$$
\left[\omega-c p-p\left(-c+\frac{\mathrm{i} \kappa}{c} \frac{d}{d p}\right)\right]\left[\omega+c p-\left(-c+\frac{\mathrm{i} \kappa}{c} \frac{d}{d p}\right) p\right] \tilde{\psi}_{\omega}=0 .
$$

Para derivar esta equação assumimos a forma aproximada para o fluxo (4.2). As soluções desta equação são

$$
\tilde{\psi}_{\omega}=A_{\omega} \theta(p) p^{-\frac{\mathrm{i} \omega c}{\kappa}-1}+B_{\omega} \theta(-p)(-p)^{-\frac{\mathrm{i} \omega c}{\kappa}-1}
$$

onde $A_{\omega}$ e $B_{\omega}$ são constantes e $\theta(x)$ é a função de Heaviside. É possível mostrar que na representação dos momenta o produto interno de Klein-Gordon, quando utilizamos (4.2), fica

$$
\left(\tilde{\psi}_{\omega}, \tilde{\psi}_{\omega^{\prime}}\right)=2 \int_{-\infty}^{\infty} d p p \tilde{\psi}_{\omega}(p) \tilde{\psi}_{\omega^{\prime}}^{*}(p)
$$

Substituindo a forma dos modos (4.5) no produto interno (4.6), obtemos a seguinte norma:

$$
\left(\tilde{\psi}_{\omega}, \tilde{\psi}_{\omega^{\prime}}\right)=\frac{4 \pi \kappa}{c}\left(\left|A_{\omega}\right|^{2}-\left|B_{\omega}\right|^{2}\right) \delta\left(\omega-\omega^{\prime}\right)
$$

Deste resultado, concluímos que os modos de norma positiva são aqueles tais que $\left|A_{\omega}\right|>$ $\left|B_{\omega}\right|$, ou seja, aqueles onde $p>0$ é predominante. 
Desejamos os coeficientes de Bogoliubov da transformação entre os modos in e os modos out. Os modos in são aqueles que tem $p>0$ e passam muito próximos ao horizonte sônico. Eles têm a forma

$$
\tilde{\psi}_{\omega}^{i n}=\sqrt{\frac{c}{4 \pi \kappa}} \theta(p) p^{-\frac{\mathrm{i} \omega c}{\kappa}-1} .
$$

Para encontrarmos os coeficientes de Bogoliubov basta calcularmos a transformada de Fourier inversa do modos. Isto, pois expandimos os modos em termos da base de ondas planas no futuro. A transformada inversa nos dá

$$
\psi_{\omega}^{i n}=\frac{1}{2 \pi} \sqrt{\frac{c}{2 \kappa}} \Gamma\left(-\frac{\mathrm{i} \omega c}{\kappa}\right)\left[\theta(z) e^{\frac{\pi \omega c}{2 \kappa}} z^{\frac{\mathrm{i} \omega c}{\kappa}}+\theta(-z) e^{-\frac{\pi \omega c}{2 \kappa}}(-z)^{\frac{\mathrm{i} \omega c}{\kappa}}\right] .
$$

A parte $z>0$ descreve os modos que sobem a correnteza e chegam até o infinito futuro, enquanto que a parte $z<0$ descreve os modos que são aprisionados do outro lado do horizonte. Em termos dos modos out, temos

$$
\psi_{\omega}^{\text {in }}=\alpha_{\omega} \psi_{\omega, \text { escapa }}^{\text {out }}+\beta_{\omega} \psi_{\omega, \text { aprisionado }}^{\text {out }} .
$$

Com isto, obtemos que a razão entre os coeficientes de Bogoliubov é

$$
\frac{\left|\beta_{\omega}\right|^{2}}{\left|\alpha_{\omega}\right|^{2}}=e^{-\frac{2 \pi \omega c}{\kappa}}
$$

e utilizando a propriedade (2.40) dos coeficientes, vem

$$
\left|\beta_{\omega}\right|^{2}=\frac{1}{e^{\frac{2 \pi \omega c}{\kappa}}-1}
$$

Logo, o horizonte de eventos emite partículas segundo um espectro térmico caracterizado pela temperatura

$$
T_{\mathrm{H}}=\frac{\kappa}{2 \pi c k_{\mathrm{B}}}
$$

\subsection{Back-reaction em análogos}

Como apontado na seção 2.2, efeitos tais como a radiação Hawking nos levam à inevitável pergunta: quando processos quânticos tomam parte, como se dá a evolução do espaçotempo? Em casos como colapso estelar, em primeira aproximação, podemos considerar que a métrica desempenha o papel de pano de fundo sobre o qual o campo quântico evolui. Podemos entender esta aproximação como linear, no sentido de que o campo não 
comparece no lado direito das equações de Einstein e, conseqüentemente, nas componentes da métrica. Na ordem seguinte, temos que levar em conta as correções na métricas devidas ao valor esperado do tensor energia-momentum do campo sobre a geometria de fundo não perturbada. Isto é o que chamamos de back-reaction. O espaço-tempo de fundo evolui e perturba o campo. Estas perturbações, por sua vez, são computadas para o conteúdo de energia do sistema e implicam em perturbações sobre a métrica do espaço-tempo.

No contexto de análogos, é perfeitamente possível nos perguntarmos: quando processos quânticos tomam parte, como se dá a evolução do fluxo de fundo? Pelo fato de as equações da hidrodinâmica serem não lineares, perturbações em primeira ordem sobre o fluxo implicarão em correções de ordem superior. Logo, para calcularmos a primeira contribuição das perturbações sonoras no meio sobre o fluxo temos de segurar termos até segunda ordem nas perturbações. Faremos isto através da expansão da ação obtida da densidade de lagrangeana (3.7).

Para um fluido ideal barotrópico cujo fluxo é irrotacional, a ação é

$$
S\left[\psi, \partial_{t} \psi, \nabla \psi, \rho\right]=\int_{t_{1}}^{t_{2}} d t \int_{V} d^{3} x\left[\rho \partial_{t} \psi-\frac{1}{2} \rho(\nabla \psi)^{2}-\rho u\right]
$$

onde $V$ é o volume ocupado pelo fluido. Suponha um estado do fluido que possa ser escrito como

$$
\begin{aligned}
& \psi=\psi_{0}+\epsilon \psi_{1}+\epsilon^{2} \psi_{2}, \\
& \rho=\rho_{0}+\epsilon \rho_{1}+\epsilon^{2} \rho_{2} .
\end{aligned}
$$

O parâmetro $\epsilon$ serve simplesmente para facilitar a separação das ordens de perturbação que aparecem ao substituirmos (4.8) em (4.7). Ele carrega o caráter de "pequeno" das perturbações $\psi_{1}, \psi_{2}, \rho_{1}$ e $\rho_{2}$, no sentido dado ao termo "pequeno" no capítulo anterior. Ao fazermos a substituição, podemos escrever a ação (4.7) como

$$
S=S_{0}+\epsilon S_{1}+\epsilon^{2} S_{2}+\epsilon^{3} S_{3}+\epsilon^{4} S_{4}
$$

A parcela $S_{0}$ é simplesmente a ação (4.7) para as soluções de fundo $\psi_{0}$ e $\rho_{0}$. Se o fluxo de fundo satisfaz as equações (4.5), então é possível mostrar que $S_{1}=0$. O termo $S_{2}$, quando variado em relação a $\psi_{1}$ e $\rho_{1}$, implica nas equações (3.8) e, ao eliminarmos $\rho_{1}$, pode ser posto na forma (2.28) se $m=0$ e a métrica tiver as componentes (3.12). Tendo fixado $\delta S_{0}=0$ e $\delta S_{2}=0$, vem que o termo $S_{3}$ é identicamente nulo. O termo $S_{4}$ tem a 
forma

$$
\begin{aligned}
S_{4}= & \int_{t_{1}}^{t_{2}} d t \int_{V} d^{3} x\left\{\rho_{2} \partial_{t} \psi_{2}+\frac{\rho_{0}}{c^{2}}\left(\partial_{t} \psi_{1}+\mathbf{v}_{0} \cdot \nabla \psi_{1}\right) \nabla \psi_{1} \cdot \nabla \psi_{2}+\rho_{2} \mathbf{v}_{0} \cdot \nabla \psi_{2}+\right. \\
& \left.-\frac{\rho_{0}}{2}\left(\nabla \psi_{2}\right)^{2}-\rho_{2}\left[\frac{1}{2}\left(\nabla \psi_{1}\right)^{2}+\frac{c^{2} \rho_{2}}{2 \rho_{0}}+\left(\partial_{t} \psi_{1}+\mathbf{v}_{0} \cdot \nabla \psi_{1}\right)^{2}\left(\frac{\rho_{0}}{2 c^{4}} \frac{d c^{2}}{d \rho_{0}}-\frac{1}{2 c^{2}}\right)\right]\right\} .
\end{aligned}
$$

Nesta expressão aparecem combinações de $\mathbf{v}_{0}$ e $\psi_{1}$ que podem ser usadas para compor componentes do tensor energia-momentum do campo $\psi_{1}$. As componentes deste tensor são

$$
T_{\mu \nu}=\partial_{\mu} \psi_{1} \partial_{\nu} \psi_{1}-\frac{1}{2} g_{\mu \nu} g^{\alpha \beta} \partial_{\alpha} \psi_{1} \partial_{\beta} \psi_{1}
$$

Seu traço e suas componentes $T_{i}^{0}$, nesta ordem, são

$$
\begin{gathered}
T_{\mu}^{\mu}=\frac{1}{\rho_{0} c}\left(\partial_{t} \psi_{1}+\mathbf{v}_{0} \cdot \nabla \psi_{1}\right)^{2}-\frac{c}{\rho_{0}}\left(\nabla \psi_{1}\right)^{2}, \\
\mathbf{T}_{i}^{0}=-\frac{1}{\rho_{0} c}\left(\partial_{t} \psi_{1}+\mathbf{v}_{0} \cdot \nabla \psi_{1}\right) \nabla \psi_{1} .
\end{gathered}
$$

Substituindo em $S_{4}$, vem

$$
\begin{aligned}
S_{4}= & \int_{t_{1}}^{t_{2}} d t \int_{V} d^{3} x\left\{\rho_{2} \partial_{t} \psi_{2}-\frac{\rho_{0}^{2}}{c} \mathbf{T}_{i}^{0} \cdot \nabla \psi_{2}+\rho_{2} \mathbf{v}_{0} \cdot \nabla \psi_{2}+\frac{\rho_{0}}{2}\left(\nabla \psi_{2}\right)^{2}+\right. \\
& \left.+\rho_{2}\left[\frac{c^{2} \rho_{2}}{2 \rho_{0}}+\left(\partial_{t} \psi_{1}+\mathbf{v}_{0} \cdot \nabla \psi_{1}\right)^{2} \frac{\rho_{0}}{2 c^{4}} \frac{d c^{2}}{d \rho_{0}}-\frac{\rho_{0}}{2} T_{\mu}^{\mu}\right]\right\} .
\end{aligned}
$$

Para simplificar, vamos supor que a velocidade do som no meio é constante. Neste caso, tomando variações de $S_{4}$ com relação a $\psi_{2}$ e $\rho_{2}$, obtemos as seguintes equações para as perturbações sobre o fluxo de fundo devidas ao som:

$$
\left\{\begin{array}{c}
\partial_{t} \psi_{2}+\mathbf{v}_{0} \cdot \nabla \psi_{2}-\frac{c^{2}}{\rho_{0}} \rho_{2}=-\frac{\rho_{0}}{2 c} T_{\mu}^{\mu} \\
\partial_{t} \rho_{2}+\nabla \cdot\left(-\rho_{0} \nabla \psi_{2}+\rho_{2} \mathbf{v}_{0}\right)=-\nabla \cdot\left(\sqrt{-g} \mathbf{T}_{i}^{0}\right)
\end{array}\right.
$$

Estamos interessados no back-reaction das flutuações quânticas sobre o fluxo de fundo. Para sermos coerentes, devemos calcular o valor médio do tensor energia-momentum no estado $|\psi\rangle$ do campo. Neste caso, temos que substituir $T_{\mu}^{\mu}$ e $\mathbf{T}_{i}^{0}$, respectivamente, por $\left\langle\hat{T}_{\mu}^{\mu}\right\rangle$ e $\left\langle\hat{\mathbf{T}}_{i}^{0}\right\rangle$ em (4.9).

Em quatro dimensões, as equações (4.9) não têm uma solução geral para uma métrica acústica arbitrária, já que é preciso saber a forma do fluxo de fundo para conhecermos as componentes do tensor energia-momentum do campo. Quando o fluxo é unidimensional, entretanto, pelo fato de qualquer espaço-tempo bidimensional ser conforme a Minkowski, é 
possível conhecer as componentes do tensor energia-momentum do campo para um fluxo qualquer. Utilizando um fluxo quase-unidimensional em um bocal de Laval, Balbinot et. al. em [33] resolveram as equações (4.9), em primeira ordem em $\hbar$, para instantes imediadamente após a formação do horizonte de eventos $(\kappa t / c \ll 1)$ e muito próximo da cintura do bocal $\left(\kappa z / c^{2} \ll 1\right)$. Como resultado, obtiveram que a posição do horizonte e a temperatura da radiação de fônons evoluem, nesta ordem, como

$$
\begin{gathered}
z_{\mathcal{H}}=-\epsilon \frac{9}{48 \pi} \frac{\kappa^{2} A_{\mathcal{H}} t}{c^{3}}, \\
T_{\mathrm{H}}=\frac{\hbar \kappa}{2 \pi c k_{\mathrm{B}}}\left(1-\epsilon \frac{563}{720 \pi} \frac{\kappa^{3} A_{\mathcal{H}} t}{c^{5}}\right) .
\end{gathered}
$$

Para tanto, utilizaram que próximo à cintura do bocal a área de secção transversal é dada aproximadamente por $A_{\mathcal{H}}\left(1-\kappa^{2} z^{2} / c^{4}\right)$, assim $A_{\mathcal{H}}$ é a área de secção transversal na cintura.

A evolução da coordenada $z$ dos pontos da superfície sobre a qual a velocidade do fluido torna-se igual a velocidade do som indica que a região subsônica avança sobre a região supersônica. Isto decorre do fato de que parte da energia do fluxo é degradada pelas partículas que deixam o horizonte. Este efeito guarda alguma semelhança com o que supõem-se ocorrer com buracos negros. Neste caso, o fluxo de partículas implica na perda de massa do objeto e na conseqüente diminuição da área do horizonte de eventos. Contudo, vemos que a evolução da temperatura do fluxo de partículas difere daquele esperado para buracos negros. Conforme a região subsônica avança sobre a região supersônica, a temperatura das partículas diminui.

Como argumentamos na seção 3.5, produzir em laboratório fluxos que simulem geometrias com um horizonte de eventos é uma tarefa experimental bastante complicada. Um outro problema, também de ordem experimental, totalmente diferente é o da detecção dos efeitos não triviais de TQCEC que podem emergir destes fluxos. Neste sentido, uma análise da ação do fluxo de partículas sobre o fluxo de fundo do fluido pode se mostrar uma janela de observação útil. 


\subsection{Modos trans-planckianos em análogos}

A TQCEC parte da hipótese de que tanto a RG como a Mecânica Quântica valem em uma vasta escala de tempo, distância e massa. De fato, alguns de seus resultados são obtidos estendendo-se seus domínios de aplicabilidade a todas as escalas destas grandezas. Entretanto, a TQCEC não passa de uma aproximação do que imaginamos ser uma teoria quântica da gravitação. Acredita-se que abaixo da escala de Planck de tempo e espaço a RG e a Mecânica Quântica não sejam mais capazes de descrever a Natureza. Logo, resultados obtidos via TQCEC que dependam de alguma forma da física trans-planckiana devem ser vistos com cautela. Este é o caso do efeito Hawking. Como mencionado na seção 2.2, os modos in, responsáveis pela presença de partículas no infinito futuro, sofrem um desvio para o vermelho arbitrariamente alto. Para que tenham comprimento de onda apreciável no futuro, estes modos, no passado, testam escalas de tempo e distância arbitrariamente pequenas, inclusive aquelas abaixo da escala de Planck. Isto nos leva à pergunta: se os modos que dão origem ao efeito Hawking testam uma física desconhecida, como podemos garantir que o espectro de partículas é de fato térmico ou mesmo ter certeza da própria existência deste fenômeno?

Segundo Jacobson [17], as divergências no UV que aparecem em teoria de campos são um bom motivo para duvidarmos de um "reservatório" de modos trans-planckianos. Uma maneira de excluir estes infinitos graus de liberdade na escala de Planck seria impor um corte nos modos com, digamos, comprimento de onda inferior a $l_{\mathrm{P}}$. Este tipo de procedimento, quando imposto aos modos in implica, devido ao desvio para o vermelho, que não teremos radiação Hawking no futuro. E não somente isto. Os modos out são extirpados dos graus de liberdade do campo; uma antena clássica não seria capaz de irradiar nestas freqüências. Um simples corte nos modos nos leva à conclusão de que simplesmente não há efeito Hawking além de outros resultados paradoxais, como o da antena clássica mencionado acima. Isto parece ser drástico demais, pois sem temperatura Hawking não temos como generalizar a segunda lei da termodinâmica para buracos negros.

Jacobson argumenta que uma forma possível de conciliar a existência da radiação Hawking e o corte em comprimento de onda abaixo da escala de Planck é supor algum mecanismo que "regenere" os modos out. Ele propõe que os modos in sejam convertidos 
em modos out de forma tal que os modos out não sofram um desvio para o vermelho arbitrariamente alto. Uma forma de produzir esta conversão seria através de uma mudança na relação de dispersão dos modos em alguma escala.

Um desvio da relação de dispersão linear implica que em algum momento temos que abrir mão da invariância de Lorentz. Vamos colocar este fato de forma mais precisa. O princípio da equivalência nos permite dizer que na vizinhança de qualquer evento de um espaço-tempo arbitrário é sempre possível construir uma família de observadores inerciais. Logo, o espaço tangente a qualquer evento do espaço-tempo da RG é invariante por transformações de Lorentz. Isto implica que, ao menos localmente, as equações dos campos podem ser colocadas na forma que têm em espaço plano desde que não acoplem com derivadas de ordem maior ou igual a dois da métrica. Então, localmente, a relação de dispersão é sempre da forma $\omega^{2}=p^{2}+m^{2}$, que é invariante sob transformações de Lorentz. Façamos agora o raciocínio inverso. Se, em alguma escala, a relação de dispersão é tal que $\omega^{2} \neq p^{2}+m^{2}$, nas vizinhanças de um ponto do espaço-tempo, então a forma da equação do campo é diferente daquela em espaço plano. Sendo assim, a equação de campo deixa de ser covariante sob transformações de Lorentz. Logo, temos que a invariância de Lorentz é quebrada; as transformações de Lorentz deixam de ser uma simetria local do espaçotempo.

O corte dos modos trans-planckianos, ou a simples mudança da relação de dispersão em alguma escala de comprimento de onda, somente é uma questão bem posta uma vez fixado um sistema de referência. Isto, pois, em princípio, através de um boost adequado com velocidade arbitrariamente próxima à da luz, somos capazes de "alongar" um modo de comprimento de onda arbitrariamente pequeno. Assim, o corte elege uma família de observadores segundo a qual dizemos quem são os modos que têm comprimento de onda abaixo da escala de Planck.

É por lançar alguma luz sobre estes problemas que os modelos análogos são um tema sedutor. Da mesma forma que em contextos astrofísicos, os modos responsáveis pela criação de fônons sofrem um desvio para o vermelho arbitrariamente alto devido a presença do horizonte acústico. Entretanto, sabemos que sistemas de matéria condensada não podem ser descritos como um contínuo em distâncias da ordem do livre caminho médio e 
em tempos da ordem do tempo livre médio das partículas no meio. Daí decorre que um fluido não consegue suportar ondas sonoras com comprimento de onda arbitrariamente curto. Nestas escalas, a equação (3.10) é inadequada para descrever perturbações no fluido. Logo, assim como com a derivação original de Hawking, temos que ter alguma cautela quanto ao resultado derivado por Unruh para a radiação de fônons emitida por buracos mudos.

No caso de sistemas gravitacionais, ainda não dispomos de uma teoria que descreva o que talvez possamos chamar de "graus de liberdade microscópicos" do espaço-tempo. Entretanto, é muito bem conhecida a física que descreve os graus de liberdade microscópicos de um sistema de matéria condensada. Em modelos análogos, então, a questão dos modos trans-planckianos (modos trans-bohrnianos talvez fosse uma denominação mais apropriada neste contexto) surge de forma mais natural e pode ser colocada de forma mais precisa: se levarmos em conta a estrutura microscópica do meio, a radiação de fônons continua tendo um espectro térmico ou mesmo continua a existir?

Uma forma através da qual a física microscópica se manifesta para as perturbações sonoras é a mudança na relação de dispersão dos modos do campo. Como apontado na seção 3.4, para ondas sonoras com comprimentos de onda suficientemente curtos a viscosidade do meio torna-se relevante e a equação para o som passa a ser a (3.18). Mostraremos no capítulo 5 que perturbações em condensados de Bose-Einstein obedecem uma relação de dispersão que difere da linear para comprimentos de onda pequenos. Em análogos, então, podemos traduzir o efeito da estrutura microscópica do meio sobre os modos trans-planckianos via uma modificação da relação de dispersão nestas escalas. Esta alteração na relação de dispersão quebra a invariância de Lorentz para as ondas sonoras. Isto é razoável, pois em escalas microscópicas é de se esperar que as simetrias do sistema de átomos (se existirem) sejam dominantes.

Para ilustrar, vamos introduzir um modelo simplificado bastante utilizado na literatura [16], [20], 18], 19] para estudar as conseqüências da alteração da relação de dispersão sobre o espectro da radiação de fônons. Considere o modelo exposto na seção 4.2. Vamos supor que a estrutura microscópica do fluido implica na seguinte modificação na equação (4.3):

$$
\left[\omega+\mathrm{i} \frac{d}{d z} \mathrm{v}_{z}\right]\left[\omega+\mathrm{iv}_{z} \frac{d}{d z}\right] \psi_{\omega}=c^{2} F^{2}\left(-\frac{d}{d z}\right) \psi_{\omega}
$$


No espaço dos momenta, temos que modificar a equação (4.4) para

$$
\left[\omega-p\left(-c+\frac{\mathrm{i} \kappa}{c} \frac{d}{d p}\right)\right]\left[\omega-\left(-c+\frac{\mathrm{i} \kappa}{c} \frac{d}{d p}\right) p\right] \tilde{\psi}_{\omega}=c^{2} F^{2}(p) \tilde{\psi}_{\omega} .
$$

No caso em que $\mathrm{v}_{z}=0$, temos a relação de dispersão no referencial do fluido,

$$
\omega^{2}=c^{2} F^{2}(p)
$$

Logo, neste modelo a família de observadores privilegiada pela relação de dispersão é aquela co-movente com o fluido. A vantagem de trabalharmos na representação do espaço de momenta reside no fato de que, enquanto (4.10) é uma equação diferencial de ordem qualquer, a equação diferencial (4.11) sempre é de ordem dois nas imediações do horizonte.

Mesmo sem impor nenhuma restrição sobre a função $F^{2}(p)$, podemos simplificar a equação (4.11). Vamos reescrevê-la como

$$
\left[\omega-c p-p\left(-c+\frac{\mathrm{i} \kappa}{c} \frac{d}{d p}\right)\right]\left[\omega+c p-\left(-c+\frac{\mathrm{i} \kappa}{c} \frac{d}{d p}\right) p\right] \tilde{\psi}_{\omega}=c^{2}\left[F^{2}(p)-p^{2}\right] \tilde{\psi}_{\omega} .
$$

Se fizermos o seguinte ansatz para a solução da equação anterior:

$$
\tilde{\psi}_{\omega}(p)=\varphi_{\omega}(p) e^{-\frac{\mathrm{i} c^{2} p}{\kappa}} \chi(p)
$$

e impusermos que $\varphi_{\omega}$ é solução da equação (4.4), vem que a função $\chi$ satisfaz

$$
\frac{d^{2} \chi}{d p^{2}}=-\frac{c^{4}}{\kappa^{2}} \frac{F^{2}(p)}{p^{2}} \chi
$$

Deste resultado fica claro o motivo pelo qual não colocamos o sub-índice $\omega$ em $\chi$. Curiosamente, $\chi$ somente depende da forma de $F$.

A mudança na forma da relação de dispersão decorre de uma mudança nas derivadas espacias. A parte que vem do acoplamento entre tempo e espaço, por conta da métrica acústica não diagonal, tem a mesma forma de antes. Logo, a forma do produto interno de Klein-Gordon é mantida. Se impusermos que os modos $\tilde{\psi}_{\omega}$ estão normalizados e que $\varphi_{\omega}$ tem a mesma norma que os modos $\tilde{\psi}_{\omega}$ na seção 4.2, vem a seguinte condição para $\chi$ :

$$
-\frac{\mathrm{i} \kappa}{2 c^{2}}\left(\chi^{*} \frac{d \chi}{d p}-\chi \frac{d \chi^{*}}{d p}\right)= \pm 1
$$

O sinal + vale para os modos de freqüência positiva enquanto que o sinal - vale para os de freqüência negativa. 
Unruh, em [16], integrou numericamente as equações deste modelo e para tanto supôs que $F(p)=p_{0}\left[\tanh \left(\frac{p}{p_{0}}\right)^{n}\right]^{\frac{1}{n}}$. O parâmetro $p_{0}$ é a escala a partir da qual manifesta-se a estrutura microscópica do meio. Como resultado, obteve que o espectro dos modos out, dentro da precisão numérica, concordava com um espectro térmico caracterizado pela temperatura de Hawking. Este resultado foi confirmado analiticamente por Brout et. al. em [20]. A confirmação do espectro de Hawking quando modificada a relação de dispersão corrobora a linha argumentativa de Jacobson, já que tais resultados, devido à relação de dispersão modificada, decorrem de mecanismos de conversão de modos. 


\section{Capítulo 5}

\section{Modelos análogos em condensados}

\section{de Bose-Einstein}

Neste capítulo discorreremos um pouco sobre a física dos condensados de Bose-Einstein. Em particular, estamos interessados numa descrição via uma teoria de campos efetiva do estado condensado quando muito próximo ao zero absoluto. Condensados de BoseEinstein são uma classe de sistemas muito interessante para construirmos espaços-tempos acústicos [26], 448], [27], [49]. Do ponto de vista experimental acredita-se que com a tecnologia disponível para manipular e realizar medições em gases ultrafrios seja possível em poucos anos construir um modelo análogo em laboratório [50], [51]. Estudos teóricos sobre análogos encontram um terreno bastante fértil em condensados já que tais sistemas têm uma física microscópica e macroscópica muito bem entendida [52], [53], [54]. Além disso, condensados de Bose-Einstein também são capazes de simular modelos cosmológicos tipo FRW [28], [29].

Na seção 5.1 mostraremos uma abordagem desenvolvida por Bogoliubov para tratar condensados de Bose-Einstein em sistemas de partículas fracamente interagentes a $T=0$. Através deste formalismo construiremos a equação de Gross-Pitaevskii, ponto de partida para a analogia. Na seção 5.2 mostraremos que, para variações da função de onda do condensado em grandes escalas, seu módulo e sua fase obedecem um par de equações acopladas que identificaremos como as equação da continuidade e de Bernoulli para um fluido ideal barotrópico. Na seção 5.3 exploraremos brevemente os vórtices quantizados 
em condensados. Na seção 5.4 construiremos os análogos cosmológicos em condensados a partir de uma nuvem de gás em expansão e da variação da secção de choque das partículas condensadas. Esta última montagem parece ser menos complicada de se implementar em laboratório. Para finalizar, apresentamos na seção 5.5 a formulação de Bogoliubov para perturbações em condensados e mostramos que esta abordagem é consistente com aquela utilizada para a construção dos análogos.

\subsection{Equação de Gross-Pitaevskii}

Considere um sistema formado por $N$ átomos bosônicos. Vamos desprezar graus de liberdade internos como spin, estrutura eletrônica e nuclear. Suponha que o sistema é suficientemente rarefeito para que somente a interação entre pares seja relevante. Do ponto de vista de segunda quantização, o hamiltoniano deste sistema de muitos corpos é

$$
\begin{aligned}
\hat{H}= & \int_{V} d^{3} x \hat{\Psi}^{\dagger}(\mathbf{x}, t)\left[-\frac{\hbar^{2}}{2 m} \nabla^{2}+\mathrm{V}(\mathbf{x})\right] \hat{\Psi}(\mathbf{x}, t)+ \\
& +\frac{1}{2} \int_{V} d^{3} x \int_{V} d^{3} x^{\prime} \hat{\Psi}^{\dagger}(\mathbf{x}, t) \hat{\Psi}^{\dagger}\left(\mathbf{x}^{\prime}, t\right) \mathrm{U}\left(\mathbf{x}-\mathbf{x}^{\prime}\right) \hat{\Psi}(\mathbf{x}, t) \hat{\Psi}\left(\mathbf{x}^{\prime}, t\right) .
\end{aligned}
$$

Aqui, $\hat{\Psi}$ é o operador campo que descreve as partículas (não-relativísticas) do sistema, $V$ é o volume ocupado pelo sistema, $\mathrm{V}$ é um potencial externo que supõe-se depender somente das coordenadas espaciais e U é o potencial de interação entre pares. O operador campo para bósons satisfaz as seguintes relações de comutação:

$$
\left[\hat{\Psi}(\mathbf{x}, t), \hat{\Psi}^{\dagger}\left(\mathbf{x}^{\prime}, t\right)\right]=\delta\left(\mathbf{x}-\mathbf{x}^{\prime}\right), \quad\left[\hat{\Psi}(\mathbf{x}, t), \hat{\Psi}\left(\mathbf{x}^{\prime}, t\right)\right]=0, \quad\left[\hat{\Psi}^{\dagger}(\mathbf{x}, t), \hat{\Psi}^{\dagger}\left(\mathbf{x}^{\prime}, t\right)\right]=0 .
$$

As relações de comutação (5.2) implicam na propriedade de que todos os bósons podem ocupar o mesmo estado ao mesmo tempo; esta propriedade está na raiz da condensação de Bose-Einstein. Na picture de Heisenberg, a equação de movimento do campo é dada por

$$
\mathrm{i} \hbar \partial_{t} \hat{\Psi}(\mathbf{x}, t)=[\hat{\Psi}(\mathbf{x}, t), \hat{H}]
$$


que implica, quando usamos as relações de comutação (5.2), na seguinte equação de movimento:

$$
\mathrm{i} \hbar \partial_{t} \hat{\Psi}=\left[\frac{-\hbar^{2}}{2 m} \nabla^{2}+\mathrm{V}+\int_{V} d^{3} x^{\prime} \hat{\Psi}^{\dagger}\left(\mathbf{x}^{\prime}, t\right) \mathrm{U}\left(\mathbf{x}-\mathbf{x}^{\prime}\right) \hat{\Psi}\left(\mathbf{x}^{\prime}, t\right)\right] \hat{\Psi}
$$

O operador número de partículas é definido como

$$
\hat{N}=\int_{V} d^{3} x \hat{\Psi}^{\dagger} \hat{\Psi}
$$

e ele é tal que $[\hat{N}, \hat{H}]=0$. Este resultado é esperado, já que a equação de Schrödinger conserva probabilidade.

Sabemos que um sistema de partículas bosônicas livres, quando resfriado a temperaturas suficientemente baixas, sofrem uma transição de fase. Abaixo da temperatura de transição o número de partículas no estado fundamental do sistema passa a ser macroscópico. A esta fase dá-se o nome de condensado de Bose-Einstein. Quanto menor a temperatura, maior o número de partículas "condensadas" no estado fundamental; a $T=0$ todas as partículas ocupam o estado de menor energia do sistema. Aqui desejamos estudar a condensação de Bose-Einstein quando as partículas não são mais livres, mas podemos considerar que a interação entre elas é pequena.

\subsubsection{Caso homogêneo}

Suponha um gás de Bose confinado em uma caixa suficientemente grande para que efeitos de borda sejam desprezíveis. Vamos considerar que o sistema não está submetido a nenhum potencial externo. Se a interação entre as partículas é pequena as ondas planas formam, em primeira aproximação, uma boa base para expandirmos o operador campo.

$$
\begin{gathered}
\hat{\Psi}(\mathbf{x}, t)=\frac{1}{V^{1 / 2}} \sum_{\mathbf{k}} e^{\mathrm{i}(\mathbf{k} \cdot \mathbf{x}-\omega t)} \hat{a}_{\mathbf{k}}, \\
\hat{\Psi}^{\dagger}(\mathbf{x}, t)=\frac{1}{V^{1 / 2}} \sum_{\mathbf{k}} e^{-\mathrm{i}(\mathbf{k} \cdot \mathbf{x}-\omega t)} \hat{a}_{\mathbf{k}}^{\dagger} .
\end{gathered}
$$

Seja $|N, 0,0, \ldots\rangle$ o estado de ocupação do estado $\mathbf{k}=\mathbf{0}$ do sistema livre. Este é o estado fundamental de um gás de Bose de partículas livres a $T=0$. Por conta das relações (5.2), os operadores $\hat{a}_{\mathbf{k}}$ e $\hat{a}_{\mathbf{k}}^{\dagger}$ satisfazem a relações de comutação (2.13). Elas implicam nas 
seguintes regras de operação sobre o estado $|N, 0,0, \ldots\rangle$ :

$$
\begin{gathered}
\hat{a}_{\mathbf{k}=\mathbf{0}}^{\dagger} \hat{a}_{\mathbf{k}=\mathbf{0}}|N, 0,0, \ldots\rangle=N|N, 0,0, \ldots\rangle, \\
\hat{a}_{\mathbf{k}=\mathbf{0}}|N, 0,0, \ldots\rangle=\sqrt{N}|N-1,0,0, \ldots\rangle, \\
\hat{a}_{\mathbf{k}=\mathbf{0}}^{\dagger}|N, 0,0, \ldots\rangle=\sqrt{N+1}|N+1,0,0, \ldots\rangle .
\end{gathered}
$$

Desejamos obter o limite termodinâmico da teoria, isto é, tomaremos $V \rightarrow \infty$ e $N \rightarrow \infty$ de forma tal que a razão $N / V$ seja finita. Estamos supondo que a interação entre as partículas é uma perturbação ao sistema. Isto significa que no limite termodinâmico o espalhamento de partículas no condensado é responsável por tirar um número ínfimo delas do estado fundamental. Como a $T=0$ o número de partículas no estado fundamental é macroscópico, convém separar o operador que cria partículas com $\mathbf{k}=\mathbf{0}$ do resto do espectro do campo. Vamos definir o seguinte operador:

$$
\hat{\xi}_{\mathbf{0}}=\frac{1}{V^{1 / 2}} \hat{a}_{\mathbf{0}}
$$

Ele satisfaz

$$
\left[\hat{\xi}_{0}, \hat{\xi}_{0}^{\dagger}\right]=\frac{1}{V}
$$

e opera sobre o estado fundamental da seguinte forma:

$$
\begin{gathered}
\hat{\xi}_{\mathbf{0}}|N, 0,0, \ldots\rangle=\left(\frac{N}{V}\right)^{1 / 2}|N-1,0,0, \ldots\rangle, \\
\hat{\xi}_{\mathbf{0}}^{\dagger}|N, 0,0, \ldots\rangle=\left(\frac{N+1}{V}\right)^{1 / 2}|N+1,0,0, \ldots\rangle .
\end{gathered}
$$

Pelas relações acima, fica claro que no limite termodinâmico os operadores $\hat{\xi}_{\mathbf{0}}$ e $\hat{\xi}_{0}^{\dagger}$ se confundem quando atuam sobre $|N, 0,0, \ldots\rangle$. Isto nos permite aproximá-los por múltiplos do operador identidade e escrevê-los como

$$
\hat{\xi}_{0}=\hat{\xi}_{0}^{\dagger}=\sqrt{n_{0}}
$$

onde $n_{0}$ é a densidade de partículas no estado fundamental. Quando $T=0$, podemos escrever o operador campo em termos da densidade de partículas no estado fundamental mais flutuações devido às interações,

$$
\hat{\Psi}=\sqrt{n_{0}}+\hat{\varphi}
$$


Daí decorre que o operador número é dado por

$$
\hat{N}=N_{0}+\sum_{\mathbf{k} \neq \mathbf{0}} \hat{a}_{\mathbf{k}}^{\dagger} \hat{a}_{\mathbf{k}}
$$

onde $N_{0}$ é o número de partículas que ocupam o estado fundamental. Este procedimento de separar o número macroscópico de partículas que ocupam o estado fundamental das excitadas implica que o operador número deixa de comutar com o hamiltoniano. Logo, o número de partículas em todo o sistema não é mais conservado, o que pode ser interpretado da maneira a seguir.

Todo o arcabouço é construído de forma tal que seja possível definir um estado de vácuo do sistema não perturbado que seja aniquilado por $\hat{a}_{\mathbf{k}}$. Como o estado fundamental é ocupado por partículas, a única forma é separar o operador $\hat{a}_{\mathbf{0}}$ do espectro do campo, pois $\hat{a}_{\mathbf{k}}|N, 0,0, \ldots\rangle=0$ para $\mathbf{k} \neq \mathbf{0}$. Isto nos permite definir o estado de vácuo $|0\rangle=$ $|N, 0,0, \ldots\rangle$. Este procedimento é lícito se estamos interessado num número macroscópico de partículas condensadas $\left(N_{0} \sim 10^{23}\right)$. Para este sistema, mais uma ou menos uma partícula no estado fundamental não implica em um estado macroscópico diferente para o condensado, mas é relevante para os estados excitados. Estas ponderações nos levam a trocarmos o operador $\hat{\xi}_{0}$ por um múltiplo da identidade, o que depois gera a inconsistência da não-conservação de partículas. Em outras palavras, a inconsistência vem do fato de aproximarmos o estado condensado por um reservatório infinito de partículas.

Sabemos, entretanto, que isto não é verdade: apesar de muito grande, o número de partículas que compõem o sistema é finito. Para contornarmos este problema, basta impormos o vínculo de que o número de partículas é constante. Seja $\left\{\left|\alpha_{i}\right\rangle\right\}$ o conjunto de auto-estados do hamiltoniano do sistema quando levamos em consideração as interações entre pares. O vínculo terá a forma

$$
N=N_{0}+\sum_{\mathbf{k} \neq \mathbf{0}}\left\langle\alpha_{0}\left|\hat{a}_{\mathbf{k}}^{\dagger} \hat{a}_{\mathbf{k}}\right| \alpha_{0}\right\rangle
$$

com $N$ sendo dado por $\left\langle\alpha_{0}|\hat{N}| \alpha_{0}\right\rangle$, onde $\left|\alpha_{0}\right\rangle$ é o estado fundamental do sistema perturbado. Impondo este vínculo, vamos reformular o problema desde o princípio. Voltemos ao ponto em que $[\hat{N}, \hat{H}]=0$. Vamos definir o seguinte operador:

$$
\hat{K}=\hat{H}-\mu \hat{N}
$$


onde $\mu$ é um parâmetro real. Este operador é hermitiano, é conservado e comuta com $\hat{N}$. Logo, tem uma base de autovetores comum com $\hat{H}$ e $\hat{N}$. O valor médio do operador $\hat{K}$ no estado de vácuo de $\hat{H}$ é

$$
\left\langle\alpha_{0}|\hat{K}| \alpha_{0}\right\rangle=K_{0}(V, N, \mu)=E_{0}(V, N)-\mu N
$$

Desejamos eliminar $N$. Se $\left|\alpha_{0}\right\rangle$ corresponde ao estado fundamental, então $E_{0}$ é o menor autovalor de $\hat{H}$. Podemos, então, fixar $\mu$ de forma tal que $K_{0}$ também seja mínimo. Esta imposição implica que

$$
\left(\frac{\partial K_{0}}{\partial N}\right)_{V, \mu}=\left(\frac{\partial E_{0}}{\partial N}\right)_{V}-\mu=0 .
$$

Logo, $\mu$ é o potencial químico do sistema a $T=0$,

$$
\mu=\left(\frac{\partial E_{0}}{\partial N}\right)_{V}
$$

Esta última é uma relação entre $N, V$ e $\mu$ através da qual podemos eliminar o número de partículas em favor de $\mu$ e $V$.

Então, para que a prescrição de trocar os operadores $\hat{\xi}_{0}$ e $\hat{\xi}_{0}^{\dagger}$ pelo escalar $\sqrt{n}$ seja consistente com um sistema de um número fixo de partículas, basta trocarmos o hamiltoniano $\hat{H}$ pelo hamiltoniano grão-canônico $\hat{K}$. Fazendo $\hat{\xi}_{0}=\hat{\xi}_{0}^{\dagger}=\sqrt{n_{0}}$, temos

$$
\begin{gathered}
\hat{N}=N_{0}+\hat{N}^{\prime}, \\
\hat{K}=E_{0}-\mu N_{0}+\hat{K}^{\prime},
\end{gathered}
$$

onde o símbolo' significa que estamos excluindo $\mathbf{k}=\mathbf{0}$ da soma sobre o espectro do operador. Até este ponto, $N_{0}$ é um parâmetro livre. Ele pode ser fixado através do potencial termodinâmico grão-canônico, definido por

$$
\Omega(T, V, \mu, N)=U-T S-\mu N
$$

impondo que $N_{0}$ é um extremo,

$$
\left[\frac{\partial}{\partial N_{0}} \Omega(T=0, V, \mu, N)\right]_{V, \mu, N=N_{0}}=0
$$

já que estamos supondo que o sistema encontra-se em equilíbrio termodinâmico. Isto implica em $N_{0}=N_{0}(\mu, V)$ e, uma vez fixado $\mu$, fixamos o número de partículas no condensado. 


\subsubsection{Caso não-homogêneo}

Vamos generalizar estes resultados para o caso em que o potencial externo é diferente de zero. Uma vez o sistema submetido a um potencial externo, não é mais útil decompormos o operador campo em termos de ondas planas. De fato, não podemos esperar que o estado fundamental do sistema seja o estado de momentum nulo, já que o potencial externo espalha as partículas. Novamente vamos supor que a interação entre pares é pequena e $T=0$. Logo, é razoável supormos que a maior parte das partículas estão no estado condensado. Isto nos permite escrever o operador campo como um campo médio $\psi$ mais flutuações,

$$
\hat{\Psi}(\mathbf{x}, t)=\psi(\mathbf{x}, t)+\hat{\varphi}(\mathbf{x}, t)
$$

onde $\psi=\langle\hat{\Psi}\rangle$. A quantidade $\psi$ é a média de ensemble do operador campo no estado condensado. No limite termodinâmico, $\psi$ é somente diferente de zero quando a temperatura do sistema está abaixo da temperatura de transição para a fase condensada. O campo médio $\psi$ é parâmetro de ordem da transição, também conhecido por função de onda do condensado.

Desejamos uma equação para $\psi$. Para tanto, lançaremos mão do hamiltoniano grãocanônico (5.7). Substituindo a decomposição do operador campo (5.8) em (5.7), é conveniente separar o hamiltoniano grão-canônico nas seguintes parcelas:

$$
\hat{K}=K_{1}+\hat{K}_{2}+\hat{K}_{3}
$$

Na parcela $K_{1}$ somente comparece o campo $\psi$. Na parcela $\hat{K}_{2}$ comparecem o campo $\psi$ e as perturbações $\hat{\varphi}$, mas estas somente até primeira ordem. Na parcela $\hat{K}_{3}$ comparecem o campo $\psi$ e as ordens maiores que a primeira das perturbações $\hat{\varphi}$. A equação de movimento para a função de onda do condensado pode ser obtida a partir da equação de Heisenberg para o operador campo $\hat{\Psi}$

$$
\mathrm{i} \hbar \partial_{t} \hat{\Psi}=\left[\hat{\varphi}, \hat{K}_{2}+\hat{K}_{3}\right]
$$

onde já utilizamos que $\left[\hat{\varphi}, K_{1}\right]=0$. Como estamos interessados na equação em ordem 
zero nas perturbações, basta calcular o comutador $\left[\hat{\varphi}, \hat{K}_{2}\right]$, sabendo que

$$
\begin{aligned}
\hat{K}_{2}= & \int_{V} d^{3} x\left\{\left[-\frac{\hbar^{2}}{2 m} \nabla^{2}+\mathrm{V}(\mathbf{x})-\mu(\mathbf{x}, t)\right] \psi^{*}(\mathbf{x}, t)\right. \\
& \left.+\psi^{*}(\mathbf{x}, t) \int_{V} d^{3} x^{\prime} \mathrm{U}\left(\mathbf{x}-\mathbf{x}^{\prime}\right)\left|\psi\left(\mathbf{x}^{\prime}, t\right)\right|^{2}\right\} \hat{\varphi}(\mathbf{x}, t) \\
& +\int_{V} d^{3} x \hat{\varphi}^{\dagger}(\mathbf{x}, t)\left\{\left[-\frac{\hbar^{2}}{2 m} \nabla^{2}+\mathrm{V}(\mathbf{x})-\mu(\mathbf{x}, t)\right]\right. \\
& \left.+\int_{V} d^{3} x^{\prime} \mathrm{U}\left(\mathbf{x}-\mathbf{x}^{\prime}\right)\left|\psi\left(\mathbf{x}^{\prime}, t\right)\right|^{2}\right\} \psi(\mathbf{x}, t),
\end{aligned}
$$

e que as perturbações $\hat{\varphi}$ satisfazem as relações de comutação (5.2). Até ordem zero, vem a seguinte equação para a função de onda do condensado:

$$
\mathrm{i} \hbar \partial_{t} \psi=\left\{\left[-\frac{\hbar}{2 m} \nabla^{2}+\mathrm{V}(\mathbf{x})-\mu(\mathbf{x}, t)\right]+\int_{V} d^{3} x^{\prime} \mathrm{U}\left(\mathbf{x}-\mathbf{x}^{\prime}\right)\left|\psi\left(\mathbf{x}^{\prime}, t\right)\right|^{2}\right\} \psi
$$

Supondo que a função de onda do condensado varie pouco na escala do raio atômico (hipótese razoável se a interação entre as partículas é pequena), podemos fazer a seguinte aproximação:

$$
\mathrm{U}\left(\mathbf{x}-\mathbf{x}^{\prime}\right)=U_{0} \delta\left(\mathbf{x}-\mathbf{x}^{\prime}\right)
$$

sendo $U_{0}$ dado por

$$
U_{0}=\frac{4 \pi a \hbar^{2}}{m}
$$

A constante positiva $a$ é o comprimento de espalhamento e está relacionada com a secção de choque das partículas. Esta aproximação implica na seguinte equação para $\psi$ :

$$
\mathrm{i} \hbar \partial_{t} \psi=\left[-\frac{\hbar}{2 m} \nabla^{2}+\mathrm{V}(\mathbf{x})-\mu(\mathbf{x}, t)+U_{0}|\psi|^{2}\right] \psi
$$

Esta é a celebrada equação de Gross-Pitaevskii. Pela seqüência de suposições que fizemos, a equação (5.10) é válida para um gás de Bose fracamente interagente a $T=0$.

\subsection{Regime hidrodinâmico}

Vamos escrever a função de onda do condensado na representação de Mandelung,

$$
\psi(\mathbf{x}, t)=\sqrt{n(\mathbf{x}, t)} e^{\frac{i}{\hbar} \chi(\mathbf{x}, t)}
$$


onde $n$ e $\chi$ são funções reais. Substituindo a forma de Mandelung na equação de GrossPitaevskii temos, nesta ordem, para a parte real e imaginária,

$$
\begin{gathered}
\partial_{t} n+\nabla \cdot\left(n \frac{\nabla \chi}{m}\right)=0, \\
\partial_{t} \chi+\frac{m}{2}\left(\frac{\nabla \chi}{m}\right)^{2}+\mathrm{V}-\mu+U_{0} n-\frac{\hbar^{2}}{2 m} \frac{\nabla^{2} \sqrt{n}}{\sqrt{n}}=0 .
\end{gathered}
$$

Por um instante, vamos reescrever a densidade de partículas como $n(\mathbf{x}, t)=n_{0} f^{2}(\mathbf{x}, t)$, onde $n_{0}$ é a densidade do sistema homogêneo e $f$ é uma função adimensional. Substituindo esta forma para $n$ em (5.12) e dividindo por $\frac{4 \pi a \hbar^{2} n_{0}}{m}$, temos que a última parcela de (5.12) torna-se

$$
-\frac{1}{8 \pi a n_{0}} \frac{\nabla^{2} f}{f}
$$

que é adimensional. O comprimento $\xi=\left(8 \pi a n_{0}\right)^{-\frac{1}{2}}$ dá uma escala de variação para a função de onda do condensado. Vamos adotar esta escala como critério para definirmos o chamado regime hidrodinâmico do condensado. O regime hidrodinâmico é obtido quando satisfeita a seguinte condição:

$$
\frac{1}{\xi^{2}} \frac{\nabla^{2} f}{f} \ll 1
$$

Neste regime podemos desprezar a última parcela da equação (5.12) e ficamos com

$$
\partial_{t}\left(\frac{\chi}{m}\right)+\frac{1}{2}\left(\frac{\nabla \chi}{m}\right)^{2}+\frac{\mathrm{V}-\mu}{m}+\frac{U_{0}}{m} n=0 .
$$

As equações (5.11) e (5.13) podem ser interpretadas, respectivamente, como a equação da continuidade e a equação de Bernoulli para um fluido ideal e barotrópico com densidade de partículas dada por $n$ e fluxo dado por $\mathbf{v}=\frac{\nabla \chi}{m}$. Esta identificação é razoável pois correntes de partículas estão relacionadas com gradientes da fase da função de onda. Note que, pelo fato de o campo de velocidades do fluido ser definido através do gradiente de um potencial escalar, o fluxo é naturalmente irrotacional. A partir do estudo das perturbações sobre um condensado não homogêneo somos capazes de construir espaçostempos acústicos da mesma forma que fizemos para um fluido ideal no capítulo 3. Aqui, as perturbações que, devido às inomogeneidades do meio, percebem uma métrica efetiva são aquelas sobre a fase da função de onda do condensado. Em particular, a velocidade do som no condensado será dada por

$$
c^{2}(\mathbf{x}, t)=\frac{U_{0} n(\mathbf{x}, t)}{m}
$$




\subsection{Vórtices quantizados}

Um resultado bastante útil diz respeito à circulação de $\mathbf{v}$ em algum caminho fechado $C$ no condensado. A circulação $\Gamma$ é definida por

$$
\Gamma=\oint_{C} \mathbf{v} \cdot d \mathbf{l}
$$

O fato de desejarmos que $\psi$ seja uma função unívoca implica que, depois de uma circuitação completa em um caminho fechado, a função $\chi$ só pode mudar de $2 \pi k \hbar$, sendo $k \in \mathbb{Z}$. Então

$$
\oint_{C} \mathbf{v} \cdot d \mathbf{l}=\frac{2 \pi k \hbar}{m}
$$

Considere uma solução das equações (5.11) e (5.13) estacionária e com simetria cilíndrica com a seguinte forma

$$
\psi(r, \theta)=\sqrt{n(r)} e^{\mathrm{i} k \theta}
$$

O fluxo correspondente é dado por

$$
\mathbf{v}=\frac{\hbar k}{m r} \hat{\theta}
$$

Supondo que não há campos externos e que $n(r \rightarrow \infty)=n_{0}$, a equação (5.13) nos dá

$$
n(r)=n_{0}-\frac{k^{2}}{8 \pi a r^{2}}
$$

O fato de a circulação em um fluido ser quantizada implica que o fluxo também o será. Isto reflete a quantização do momentum angular do fluido. Esta peculiaridade deste fluido quântico poderia ser usada para imprimir à radiação Hawking gerada uma característica especial. Uma montagem através da qual isto poderia ser implementado seria um condensado escoando em um toro que em alguma região afunila, formando um bocal de Laval.

\subsection{Modelos cosmológicos em condensados}

\subsubsection{Condensados em expansão}

Considere um condensado de Bose-Einstein que está confinado em uma armadilha estática para $t<0$. Em $t=0$ o potencial aprisionante é desligado e a nuvem de gás pode expandir 
livremente. Supondo que a nuvem seja esfericamente simétrica, é possível mostrar que seu raio evolui segundo a seguinte equação [51]

$$
r(t)=r_{0} b(t)
$$

onde $r_{0}$ é a posição radial inicial de cada ponto da amostra se $b(t=0)=1$. O volume do condensado é dado por

$$
V(t)=\frac{4}{3} \pi R^{3}(t)=V(0) b^{3}(t)
$$

onde $R_{0}$ é o raio da amostra, e a velocidade com que a nuvem expande será

$$
\mathrm{v}_{r}(t)=\dot{r}(t)=r(t) \frac{\dot{b}(t)}{b(t)}
$$

Supondo que a nuvem é homogênea, a velocidade do som no meio pode ser escrita como

$$
c^{2}(\mathbf{x}, t)=\frac{c^{2}(\mathbf{x}, 0)}{b^{3}(t)}
$$

já que $V(t)=V(0) b^{3}(t)$.

Tendo em vista estes resultados, o elemento de linha do espaço-tempo acústico,

$$
d s^{2}=\frac{n}{c}\left[-\left(c^{2}-\mathrm{v}_{r}^{2}\right) d t^{2}-2 \mathrm{v}_{r} d r d t+d r^{2}+r^{2} d \Omega^{2}\right],
$$

pode ser colocado na forma

$$
d s^{2}=\frac{n(\mathbf{x}, 0)}{c(\mathbf{x}, 0)}\left[-c^{2}(\mathbf{x}, 0) b^{-9 / 2} d t^{2}+b^{1 / 2}\left(d r_{0}^{2}+r_{0}^{2} d \Omega^{2}\right)\right]
$$

Definindo a seguinte coordenada temporal:

$$
d \tau=b^{-9 / 4}(t) d t
$$

vem que

$$
d s^{2}=\frac{n(\mathbf{x}, 0)}{c(\mathbf{x}, 0)}\left[-c^{2}(\mathbf{x}, 0) d \tau^{2}+b^{1 / 2}\left(d R_{0}^{2}+R_{0}^{2} d \Omega^{2}\right)\right] .
$$

Desta forma, para o elemento de linha do espaço-tempo acústico vemos que uma nuvem com densidade uniforme é capaz de simular um espaço-tempo homogêneo e isotrópico em expansão. Neste caso, $\tau$ é a coordenada temporal análoga a dos observadores co-moventes com o fluido cósmico. 
Em particular, no caso em que $b(t)=(9 H t+1)^{4 / 9}$, temos que a relação entre as coordenadas temporais $t$ e $\tau$ é

$$
t(\tau)=\frac{1}{9 H}\left[e^{9 H\left(\tau-\tau_{0}\right)}-1\right]
$$

onde $H$ é uma constante. Logo, a função $b$, como função de $\tau$, é dada por

$$
b(\tau)=e^{4 H\left(\tau-\tau_{0}\right)}
$$

se tomarmos $b\left(\tau_{0}\right)=1$. Neste caso, temos um análogo ao universo de de Sitter.

Apesar de aparentemente mais simples de realizar em laboratório, já que prescindem de fluxos supersônicos, os análogos cosmológicos usando nuvens em expansão também têm algumas limitações. Uma delas decorre do fato de que a equação de movimento para os pontos da nuvem é obtida ao desprezarmos o termo da energia cinética na equação de Gross-Pitaevskii [29]. Conforme o tempo passa, a energia cinética do gás, em detrimento da energia potencial, aumenta e em algum momento torna-se comparável à energia de interação entre as partículas. Isto limita a validade da equação (5.15) somente para instantes próximos a $t=0$. Logo, através de nuvens de gás em expansão somos capazes de simular um universo em expansão por um certo intervalo de tempo.

\subsubsection{Expansão via variação da secção de choque}

Uma forma mais simples de implementarmos um análogo cosmológico em condensados é através da variação da velocidade do som no meio [28]. Considere um condensado descrito por uma função de onda $\psi$ tal que $n$ é constante e homogêneo. Neste caso, o elemento de linha do espaço-tempo acústico tem a forma

$$
d s^{2}=-c(t) d t^{2}+\frac{1}{c(t)} d \mathbf{x}^{2}
$$

Redefinindo a coordenada temporal como

$$
d \tau=c^{1 / 2}(t) d t
$$

vem que

$$
d s^{2}=-d \tau^{2}+\frac{1}{c(t)} d \mathbf{x}^{2}
$$


Logo, o parâmetro de expansão é o fator $1 / c(t)$. Note que quanto menor a velocidade do som maior o raio do universo. Isto ocorre pois, do ponto de vista do laboratório, quanto menor a velocidade do som mais tempo leva para observadores comunicarem-se através de sinais sonoros. Para estes observadores hipotéticos, então, tudo se passa como se a velocidade do som se mantivesse a mesma mas as distâncias espaciais tivessem aumentado. Logo, eles têm a impressão de que o universo em que vivem "inchou".

Em laboratório, é possível manipular a velocidade do som em um condensado de Bose-Einstein. A velocidade do som no meio é dada por

$$
c^{2} \propto a
$$

isto é, $c^{2}$ é proporcional ao comprimento de espalhamento das partículas que compõem o gás. Logo, se for possível manipular o parâmetro $a$ de forma que ele decresça no tempo, então é possível simular um universo em expansão sem alterar as dimensões da nuvem de gás.

Para que ao longo de todo o processo possamos definir um espaço-tempo acústico é necessário que a equação de Gross-Pitaevskii valha a todo instante. Logo, é imperativo que a escala de tempo em que o parâmetro $a$ é manipulado seja consistente com as aproximações que levaram à equação (5.10). Além disto, é necessário que as partículas, após a colisão, não carreguem a informação de que sua secção de choque está mudando; num certo sentido, é necessário que o processo de espalhamento seja markoviano. Se carregarem esta informação, teremos uma equação tipo Gross-Pitaevskii com um termo a mais descrevendo a colisão através de uma integral no tempo. Tipicamente, esta integral teria uma contribuição relevante em janelas de tempo da ordem da duração da colisão entre as partículas. Para que este termo seja desprezível, é necessário que a escala em que a secção de choque das partículas muda seja muito maior que a duração de uma colisão.

Para estimar a ordem da duração de uma colisão, basta sabermos o tamanho da região em que a interação entre os átomos é relevante e qual a velocidade típica das partículas. Para átomos alcalinos, a região em que a interação ocorre é da ordem de $10^{-9} \mathrm{~m}$. O momentum típico dos átomos pode ser estimado através do princípio da incerteza, já que eles se encontram confinados em alguma armadilha. Para armadilhas com comprimento da ordem de $10^{-5} \mathrm{~m}$, temos que $p \sim 10^{-29} \mathrm{~kg} \times \mathrm{m} / \mathrm{s}$ e, para massas da ordem de $10^{-27} \mathrm{~kg}$, 
temos que $v=p / m \sim 10^{-2} \mathrm{~m} / \mathrm{s}$. Logo, a escala de tempo da interação entre pares é da ordem de $10^{-7} \mathrm{~s}$. Temos, então, que variar a secção de choque dos átomos numa escala maior que décimos de microssegundos para que a hipótese de que a colisão é markoviana seja válida.

\subsection{Teoria de Bogoliubov para excitações em conden- sados}

Para que o tratamento das perturbações em condensados seja coerente, é necessário verificar se a teoria de Bogoliubov coincide com o tratamento perturbativo via a equação de Gross-Pitaevskii [55]. Para tratar as perturbações térmicas de um condensado de Bose-Einstein a temperaturas próximas do zero absoluto, N. N. Bogoliubov desenvolveu o formalismo abordado na seção 5.1 e aplicou a transformação que leva seu nome para diagonalizar o hamiltoniano perturbado. Vejamos como.

Novamente, considere um sistema de muitos átomos bosônicos descritos pelo hamiltoniano grão-canônico (5.7) com a interação entre pares dada por (5.9). Supondo que o sistema não está submetido a um potencial externo V, é útil fazer a decomposição em ondas planas (5.5) do campo $\hat{\Psi}$ e separar os operadores que criam e aniquilam partículas no estado de momentum nulo. Se a temperatura for suficientemente baixa para que o número de partículas no estado condensado seja macroscópico e muito maior que o número de átomos nos estados excitados, então podemos tratar os operadores $\hat{\xi}_{\mathbf{0}}$ e $\hat{\xi}_{\mathbf{0}}^{\dagger}$ como múltiplos da identidade. Comparando a expansão (5.5) com a decomposição (5.6) concluímos que

$$
\hat{\varphi}(\mathbf{x}, t)=\frac{1}{V^{1 / 2}} \sum_{\mathbf{k} \neq \mathbf{0}} e^{i \mathbf{k} \cdot \mathbf{x}} \hat{a}_{\mathbf{k}}(t) .
$$

Substituindo a decomposição (5.6) no hamiltoniano grão-canônico e novamente o separando em parcelas, vem que

$$
K_{1}=-\frac{U_{0} V n_{0}^{2}}{2}
$$




$$
\begin{aligned}
\hat{K}_{3} & =\int_{V} d^{3} x \hat{\varphi}^{\dagger}(\mathbf{x}, t)\left[-\frac{\hbar^{2}}{2 m} \nabla^{2}-\mu\right] \hat{\varphi}(\mathbf{x}, t)+ \\
& +U_{0} \int_{V} d^{3} x\left[\frac{1}{2} \hat{\varphi}^{\dagger}(\mathbf{x}, t) \hat{\varphi}^{\dagger}(\mathbf{x}, t)+\frac{1}{2} \hat{\varphi}(\mathbf{x}, t) \hat{\varphi}(\mathbf{x}, t)+2 \hat{\varphi}^{\dagger}(\mathbf{x}, t) \hat{\varphi}(\mathbf{x}, t)\right] .
\end{aligned}
$$

A parcela $K_{1}$ é obtida quando satisfazemos a equação de Gross-Pitaevskii para $n$ constante e potencial externo nulo. A parcela $\hat{K}_{2}$ é identicamente nula. Substituindo a expansão em ondas planas das flutuações em $\hat{K}_{3}$ e integrando em todo o espaço, temos que o hamiltoniano grão-canônico torna-se

$$
\hat{K}=-\frac{U_{0} V n_{0}^{2}}{2}+\sum_{\mathbf{k} \neq \mathbf{0}}\left(\epsilon_{\mathbf{k}}^{0}+U_{0} n_{0}\right) \hat{a}_{\mathbf{k}}^{\dagger} \hat{a}_{\mathbf{k}}+U_{0} n_{0} \sum_{\mathbf{k} \neq \mathbf{0}}\left(\frac{1}{2} \hat{a}_{\mathbf{k}}^{\dagger} \hat{a}_{-\mathbf{k}}^{\dagger}+\frac{1}{2} \hat{a}_{\mathbf{k}} \hat{a}_{-\mathbf{k}}\right),
$$

$\operatorname{com} \epsilon_{\mathrm{k}}^{0}$ dado por

$$
\epsilon_{\mathrm{k}}^{0}=\frac{\hbar^{2} \mathrm{k}^{2}}{2 m}
$$

Para diagonalizar o operador $\hat{K}$, utilizaremos a seguinte transformação de Bogoliubov:

$$
\begin{aligned}
& \hat{a}_{\mathbf{k}}=u_{\mathrm{k}} \hat{b}_{\mathbf{k}}-v_{\mathrm{k}} \hat{b}_{-\mathbf{k}}^{\dagger}, \\
& \hat{a}_{\mathbf{k}}^{\dagger}=u_{\mathrm{k}} \hat{b}_{\mathbf{k}}^{\dagger}-v_{\mathrm{k}} \hat{b}_{-\mathbf{k}},
\end{aligned}
$$

impondo que os operadores $\hat{b}_{\mathbf{k}}$ e $\hat{b}_{\mathbf{k}}^{\dagger}$ satisfazem as mesmas relações de comutação que os operadores $\hat{a}_{\mathbf{k}}$ e $\hat{a}_{\mathbf{k}}^{\dagger}$ e que os coeficientes $u_{\mathrm{k}}$ e $v_{\mathrm{k}}$ sejam reais. Para que ambas as relações de comutação $\left[\hat{a}_{\mathbf{k}}, \hat{a}_{\mathbf{k}^{\prime}}^{\dagger}\right]=\delta_{\mathbf{k}, \mathbf{k}^{\prime}}$ e $\left[\hat{b}_{\mathbf{k}}, \hat{b}_{\mathbf{k}^{\prime}}^{\dagger}\right]=\delta_{\mathbf{k}, \mathbf{k}^{\prime}}$ sejam simultaneamente satisfeitas, é necessário que os coeficientes $u_{\mathrm{k}}$ e $v_{\mathrm{k}}$ satisfaçam

$$
u_{\mathrm{k}}^{2}-v_{\mathrm{k}}^{2}=1
$$

As outras duas relações não implicam em nenhuma restrição sobre os coeficientes $u_{\mathrm{k}} \mathrm{e}$ $v_{\mathrm{k}}$. Substituindo a transformação de Bogoliubov em $\hat{K}$, temos que se os coeficientes da transformação tiverem a forma

$$
\begin{aligned}
& u_{\mathrm{k}}^{2}=\frac{1}{2}\left\{1+\frac{\epsilon_{\mathrm{k}}^{0}+U_{0} n_{0}}{\left[\epsilon_{\mathrm{k}}^{0}\left(\epsilon_{\mathrm{k}}^{0}+2 U_{0} n_{0}\right)\right]^{1 / 2}}\right\}, \\
& v_{\mathrm{k}}^{2}=\frac{1}{2}\left\{-1+\frac{\epsilon_{\mathrm{k}}^{0}+U_{0} n_{0}}{\left[\epsilon_{\mathrm{k}}^{0}\left(\epsilon_{\mathrm{k}}^{0}+2 U_{0} n_{0}\right)\right]^{1 / 2}}\right\},
\end{aligned}
$$

então o hamiltoniano grão-canônico reduz-se a

$$
\hat{K}=-\frac{U_{0} V n_{0}^{2}}{2}-\frac{1}{2} \sum_{\mathbf{k} \neq \mathbf{0}}\left(\epsilon_{\mathrm{k}}^{0}+U_{0} n_{0}-\epsilon_{\mathrm{k}}\right)+\sum_{\mathbf{k} \neq \mathbf{0}} \epsilon_{\mathrm{k}} \hat{b}_{\mathbf{k}}^{\dagger} \hat{b}_{\mathbf{k}}
$$


com

$$
\epsilon_{\mathrm{k}}=\sqrt{\frac{\hbar^{2} \mathrm{k}^{2}}{2 m}\left(\frac{\hbar^{2} \mathrm{k}^{2}}{2 m}+2 U_{0} n_{0}\right)} .
$$

A relação de dispersão (5.17) é conhecida por relação de dispersão de Bogoliubov. Ela apresenta dois limites interessantes. Vamos chamar de modos de comprimento de onda longo aqueles tais que

$$
\lambda \gg \frac{h}{\sqrt{4 U_{0} n_{0} m}} .
$$

Para eles a relação (5.17) reduz-se a

$$
\omega_{\mathrm{k}}=c \mathrm{k}
$$

com $c$, a velocidade do som, dada por (5.14). Os modos de comprimento de onda curtos são aqueles tais que

$$
\lambda \ll \frac{\hbar}{\sqrt{4 U_{0} n_{0} m}},
$$

e neste caso

$$
\omega_{\mathrm{k}}=\frac{\hbar \mathrm{k}^{2}}{2 m}
$$

Os modos de comprimento de onda longos descrevem partículas "relativísticas" sem massa. Estes são os fônons: modos coletivos das partículas do sistema. Os modos de comprimento de onda curtos descrevem partículas newtonianas de massa $m$. Estas são excitações sobre o estado condensado sensíveis aos graus de liberdade microscópicos do sistema. Note que a escala de comprimento definida pela relação de dispersão de Bogoliubov é a mesma escala de comprimento que emerge da equação de Gross-Pitaevskii quando definimos o regime hidrodinâmico. Logo, no contexto da teoria de Bogoliubov e no contexto da aproximação hidrodinâmica os fônons emergem na mesma escala de comprimento.

Pelas regras de comutação, sabemos que o operador $\hat{b}_{\mathbf{k}}^{\dagger} \hat{b}_{\mathbf{k}}$, o operador número das chamadas quase-partículas, tem como autovalores os números naturais. O autovetor com autovalor nulo é aquele definido por

$$
\hat{b}_{\mathbf{k}}|\tilde{0}\rangle=0
$$

para $\mathbf{k} \neq \mathbf{0}$. Da mesma forma, o autovetor de autovalor nulo de $\hat{a}_{\mathbf{k}}^{\dagger} \hat{a}_{\mathbf{k}}$ é definido por

$$
\hat{a}_{\mathbf{k}}|0\rangle=0,
$$


também para $\mathbf{k} \neq \mathbf{0}$. Se aplicarmos o operador $\hat{a}_{\mathbf{k}}$ no vetor $|\tilde{0}\rangle$ vem que

$$
\hat{a}_{\mathbf{k}}|\tilde{0}\rangle=\left(u_{\mathrm{k}} \hat{b}_{\mathbf{k}}-v_{\mathrm{k}} \hat{b}_{-\mathbf{k}}^{\dagger}\right)|\tilde{0}\rangle=-v_{\mathrm{k}}\left|\tilde{1}_{-\mathbf{k}}\right\rangle
$$

Repare que no limite de comprimentos de onda curtos, quando podemos desprezar $U_{0} n_{0}$ frente a $\epsilon_{\mathrm{k}}^{0}$, temos que o coeficiente de Bogoliubov $v_{\mathrm{k}}$ é nulo. Neste limite o vácuo do hamiltoniano microscópico e o vácuo das quase-partículas se confundem. Por conta disto, podemos interpretar as partículas newtonianas que emergem neste limite como átomos em estados excitados. Como $\hat{a}_{\mathbf{k}}$ não aniquila o estado $|\tilde{0}\rangle$, temos que os auto-estados de $\hat{a}_{\mathbf{k}}^{\dagger} \hat{a}_{\mathbf{k}}$ são combinações não triviais dos auto-estados de $\hat{b}_{\mathbf{k}}^{\dagger} \hat{b}_{\mathbf{k}}$. Em particular, o auto-estado $|\tilde{0}\rangle$ implica no seguinte número de partículas fora do condensado:

$$
\left\langle\tilde{0}\left|\hat{a}_{\mathbf{k}}^{\dagger} \hat{a}_{\mathbf{k}}\right| \tilde{0}\right\rangle=v_{\mathrm{k}}^{2}
$$

para cada estado $\mathbf{k} \neq \mathbf{0}$. Note que se $U_{0}=0$, então o número de partículas nos estados excitados é nulo. A energia do sistema no estado de vácuo das quase-partículas é

$$
\langle\tilde{0}|\hat{H}| \tilde{0}\rangle=\frac{U_{0} V n_{0}^{2}}{2}-\frac{1}{2} \sum_{\mathbf{k} \neq \mathbf{0}}\left(\epsilon_{\mathrm{k}}^{0}+U_{0} n_{0}-\epsilon_{\mathrm{k}}\right),
$$

que evidentemente diverge. Isto pode ser corrigido levando em conta termos de maior ordem nos elementos da matriz do potencial de pares. Novamente notamos que se $U_{0}=0$, a energia do estado fundamental das quase-partículas é nula.

Desejamos relacionar as quase-partículas do formalismo de Bogoliubov com os fônons encontrados via perturbação da equação de Gross-Pitaevskii. Podemos escrever o operador campo $\hat{\Psi}$ como a função de onda do condensado mais flutuações quânticas sobre a solução de fundo,

$$
\hat{\Psi}=\sqrt{n_{0}+\hat{n}_{1}} e^{\mathrm{i}\left(\chi_{0}+\hat{\chi}_{1}\right)} \simeq \sqrt{n_{0}} e^{\mathrm{i} \chi_{0}}+\sqrt{n_{0}} e^{\mathrm{i} \chi_{0}}\left(\frac{\hat{n}_{1}}{2 n_{0}}+\mathrm{i} \hat{\chi}_{1}\right)
$$

Daí concluímos que podemos expressar o operador campo de perturbações como

$$
\begin{gathered}
\hat{\varphi}=\sqrt{n_{0}} e^{\mathrm{i} \chi_{0}}\left(\frac{\hat{n}_{1}}{2 n_{0}}+\mathrm{i} \hat{\chi}_{1}\right), \\
\hat{\varphi}^{\dagger}=\sqrt{n_{0}} e^{-\mathrm{i} \chi_{0}}\left(\frac{\hat{n}_{1}^{\dagger}}{2 n_{0}}-\mathrm{i} \hat{\chi}_{1}^{\dagger}\right),
\end{gathered}
$$


e se os operadores $\hat{n}_{1}$ e $\hat{\chi}_{1}$ são hermitianos, podemos inverter as relações acima,

$$
\begin{gathered}
\hat{n}_{1}=\sqrt{n_{0}} e^{i \chi_{0}}\left(\hat{\varphi}^{\dagger}+\hat{\varphi}\right), \\
\hat{\chi}_{1}=\frac{i e^{-i \hat{\chi}_{0}}}{2 \sqrt{n_{0}}}\left(\hat{\varphi}^{\dagger}-\hat{\varphi}\right) .
\end{gathered}
$$

A partir das relações de comutação (5.2) para o operador $\hat{\varphi}$ vem que os operadores $\hat{n}_{1}$ e $\hat{\chi}_{1}$ satisfazem

$$
\left[\hat{n}_{1}(\mathbf{x}, t), \hat{\chi}_{1}\left(\mathbf{x}^{\prime}, t\right)\right]=\mathrm{i} \delta\left(\mathbf{x}-\mathbf{x}^{\prime}\right)
$$

Deste resultado podemos concluir que o operador $\hat{\chi}_{1}$ é o momentum canonicamente conjugado do operador $\hat{n}_{1}$. Logo, os operadores de criação e destruição dos modos destes campos satisfazem as relações (2.13). Isto mostra que perturbar a equação de GrossPitaevskii e estudar as flutuações quânticas através de um campo bosônico é consistente com a teoria que descreve os graus de liberdade microscópicos do sistema. 


\section{Capítulo 6}

\section{Outros modelos}

Neste capítulo discorreremos brevemente sobre geometrias efetivas que emergem em contextos um pouco diferentes dos abordados nos capítulos anteriores. Na seção 6.1 trataremos de uma proposta de R. Schültzhold e W. G. Unruh [56] de modelo análogo baseado em ondas na superfície de um fluido ideal cujo fluxo é irrotacional. Apesar de ainda ser baseado nas equações da hidrodinâmica, este modelo tem a vantagem de a velocidade do som no meio estar ligada à profundidade do canal em que o fluido escoa o que a torna um parâmetro de fácil manipulação experimental. Na seção 6.2 mostraremos que pequenas oscilações em cordas tensionadas são capazes de simular espaços-tempos de $1+1$ dimensões [57]. Eles são interessantes, pois são capazes de representar espaços-tempos de $3+1$ dimensões. Por último, na seção 6.3, mostraremos que é um fato bastante geral que sistemas cuja equação de movimento pode ser obtida via o formalismo lagrangeano dêem origem a geometrias efetivas no processo de linearização [58].

\subsection{Ondas em superfícies de fluidos}

Considere um fluido ideal e incompressível que escoa em um canal ou canaleta. Suponha que o fluido está submetido ao campo gravitacional g nas imediações da superfície da Terra e sua superfície livre suporta uma pressão externa $P_{\text {ext }}$. Vamos tomar o fundo do canal como plano e paralelo à superfície do fluido não perturbado e que o sistema de coordenadas é tal que $\hat{\mathbf{z}}$ é o versor normal a este plano. Se o fluxo é irrotacional, as 
equações de movimento são

$$
\begin{aligned}
-\partial_{t} \psi+\frac{1}{2}(\nabla \psi)^{2} & =-\frac{P}{\rho}-\mathrm{g} z-\mathrm{V}_{\|}, \\
\nabla^{2} \psi & =0
\end{aligned}
$$

na ausência de fontes. $\mathrm{O}$ potencial $\mathrm{V}_{\|}$é tal que a densidade de força $\mathbf{f}_{\|}=-\rho \nabla \mathrm{V}_{\|}$não tem componente na direção $\hat{\mathbf{z}}$. Seja $h(x, y, t)$ a altura dos pontos pertencentes à superfície livre do fluido num certo instante $t$. Suponha que o fundo do canal pertença ao plano $z=0$. A este problema devemos impor as seguintes condições de contorno:

$$
\begin{gathered}
\mathrm{v}_{z}(x, y, z=0, t)=0, \\
P(x, y, z=h, t)=P_{\text {ext }} \\
\mathrm{v}_{z}(x, y, z=h, t)=\partial_{t} h+\mathbf{v} \cdot \nabla h .
\end{gathered}
$$

Suponha um estado do fluido que possa ser expresso em termos de uma solução de fundo mais perturbações,

$$
\begin{aligned}
& \mathbf{v}=\mathbf{v}_{0}+\mathbf{v}_{1}, \\
& P=P_{0}+P_{1}, \\
& h=h_{0}+h_{1} .
\end{aligned}
$$

Ao fluxo de fundo vamos impor que $\mathrm{v}_{0 z}=0$ e $(\hat{\mathbf{z}} \cdot \nabla) \mathbf{v}_{0}=\mathbf{0}$. Isto implica que o fluxo de fundo é puramente horizontal, isto é, $\mathbf{v}_{0}=\mathbf{v}_{0}^{\|}$e homogêneo ao longo da direção $\hat{\mathbf{z}}$. Logo, $h_{0}$, a altura da superfície livre o fluido não perturbada, é constante. A condição de que o escoamento é puramente horizontal implica, através da equação de Euler, que

$$
P_{0}=\rho \mathrm{g}\left(h_{0}-z\right)+P_{\text {ext }}
$$

Até primeira ordem, as equações para as perturbações são

$$
\begin{gathered}
-\partial_{t} \psi_{1}+\mathbf{v}_{0} \cdot \nabla \psi_{1}=-\frac{P_{1}}{\rho}, \\
\nabla^{2} \psi_{1}=0 .
\end{gathered}
$$

Da condição de contorno (6.1) vem que

$$
-\hat{\mathbf{z}} \cdot \nabla \psi_{1}\left(x, y, z=h_{0}, t\right)=\partial_{t} h_{1}+\mathbf{v}_{0} \cdot \nabla h_{1} .
$$


Em primeira ordem, as perturbações na pressão e na altura dos pontos da superfície livre do fluido estão relacionadas por

$$
P_{1}=\rho \mathrm{g} h_{1}
$$

Substituindo (6.5) em (6.2), podemos escrever $h_{1}$ em termos de $\psi_{1}$,

$$
h_{1}=\frac{1}{\mathrm{~g}}\left(\partial_{t} \psi_{1}+\mathbf{v}_{0} \cdot \nabla \psi_{1}\right)
$$

Desejamos obter uma equação para as ondulações da superfície livre do fluido em termos das variáveis $x, y$ e $t$ e suas derivadas. Para tanto, temos que eliminar a derivada em $z$ em (6.4). Isto é possível se integrarmos a equação da continuidade para as perturbações (6.3) em z. Temos

$$
-\hat{\mathbf{z}} \cdot \nabla \psi_{1}=\nabla_{\|}^{2} \int^{z} d z^{\prime} \psi_{1}\left(x, y, z^{\prime}, t\right) .
$$

É útil expandirmos o potencial de velocidades em torno da superfície livre do fluido,

$$
\psi_{1}=\left.\sum_{n=0}^{\infty} \frac{\left(z-h_{0}\right)^{n}}{n !} \psi_{1}^{(n)}\right|_{z=h_{0}} .
$$

Vamos considerar o limite de águas rasas, isto é, $\lambda \gg h_{0}$. Como $\left(z-h_{0}\right)^{n} \sim h_{0}^{n}$ e $\nabla_{\|}^{2} \sim \lambda^{-2}$, podemos desprezar as parcelas com termos $h_{0} / \lambda$ maiores que zero quando tomamos $\nabla_{\|}^{2}$ da expansão (6.7). Logo, neste limite é lícito aproximarmos $\nabla_{\|}^{2} \psi_{1}(x, y, z, t)$ por $\nabla_{\|}^{2} \psi_{1}\left(x, y, z=h_{0}, t\right)$ nas imediações da superfície livre do fluido. Por fim, temos que

$$
-\hat{\mathbf{z}} \cdot \nabla \psi_{1}\left(x, y, z=h_{0}, t\right)=\nabla_{\|}^{2} \psi_{1}^{(0)}\left(x, y, z=h_{0}, t\right)
$$

Voltando este resultado em (6.4) obtemos que

$$
h_{0} \nabla_{\|}^{2} \psi_{1}^{(0)}=\partial_{t} h_{1}+\mathbf{v}_{0} \cdot \nabla h_{1}
$$

No limite de águas rasas, podemos utilizar (6.8) para eliminar $h_{1}$ em (6.6) e obter seguinte equação para $\psi_{1}^{(0)}$ :

$$
\left(\partial_{t}+\mathbf{v}_{0}^{\|} \cdot \nabla_{\|}\right)^{2} \psi_{1}^{(0)}-h_{0} \mathrm{~g} \nabla_{\|}^{2} \psi_{1}^{(0)}=0
$$

Esta equação pode ser colocada na forma (3.14), onde as componentes do tensor métrico inverso do espaço-tempo acústico são

$$
g^{\mu \nu}=\left[\begin{array}{ccc}
-1 & -\mathrm{v}_{0 x}^{\|} & -\mathrm{v}_{0 y}^{\|} \\
-\mathrm{v}_{0 x}^{\|} & c^{2}-\mathrm{v}_{0 x}^{\| 2} & -\mathrm{v}_{0 x}^{\|} \mathrm{v}_{0 y}^{\|} \\
-\mathrm{v}_{0 y}^{\|} & -\mathrm{v}_{0 x}^{\|} \mathrm{v}_{0 y}^{\|} & c^{2}-\mathrm{v}_{0 y}^{\| 2}
\end{array}\right],
$$


onde $c$ é a velocidade do som no meio e é dada por

$$
c^{2}=h_{0} \text { g. }
$$

Este modelo tem vantagens sobre o modelo em fluidos apresentado no capítulo 3 , Em primeiro lugar, a velocidade de fase das ondas depende de um parâmetro facilmente manipulado em laboratório: a profundidade do canal. Isto permite construir espaçostempos acústicos dotados de um horizonte de eventos sem que seja necessário que o escoamento dê-se a uma velocidade próxima a velocidade do som no bulk do fluido. Assim, podemos evitar a formação de ondas de choque no meio. Outra vantagem é a de que as ondas podem ser detectadas através da variação da altura da superfície, o que permite técnicas simples de medida tais como interferometria. Por outro lado, segundo Schützhold e Unruh [56], este modelo não é adequado para a verificação de efeitos quânticos pois detectá-los torna-se ainda mais difícil devido à baixa velocidade das ondas. Ainda assim, seria um modelo útil para simularmos efeitos de teoria clássica de campos em espaços curvos como, por exemplo, a superradiância e a mistura de modos de norma positiva e negativa.

Até este ponto consideramos que a força que atua no fluido está ligada ao transporte de momentum via pressão interna. Na superfície do fluido, entretanto, a tensão superficial torna-se importante quando sua curvatura torna-se suficientemente grande. A tensão superficial está ligada a forças que em primeira aproximação podem ser consideradas elásticas e surgem da interação entre os átomos do fluido.

Em uma membrana elástica a força restauradora é proporcional ao laplaciano de suas deformações. Disto podemos concluir que, para levar em conta a tensão superficial, devemos modificar a condição de contorno para a pressão na superfície livre do fluido para

$$
P(x, y, z=h, t)=P_{\mathrm{ext}}-\alpha \nabla_{\|}^{2} h
$$

onde $\alpha$ denota o coeficiente de tensão superficial do fluido, um parâmetro real e constante. Isto implica que a relação entre $P_{1}$ e $h_{1}$ é modificada para

$$
P_{1}=\rho g h_{1}-\alpha \nabla_{\|}^{2} h_{1}
$$

e voltando na equação de Bernoulli para as perturbações, temos

$$
\mathrm{g} h_{1}-\frac{\alpha}{\rho} \nabla_{\|}^{2} h_{1}=\partial_{t} \psi_{1}+\mathbf{v}_{0} \cdot \nabla \psi_{1} .
$$


Tomando o operador $\left(\partial_{t}+\mathbf{v}_{0} \cdot \nabla\right)$ da equação acima vem que

$$
\mathrm{g}\left(\partial_{t}+\mathbf{v}_{0} \cdot \nabla\right) h_{1}-\frac{\alpha}{\rho}\left(\partial_{t}+\mathbf{v}_{0} \cdot \nabla\right) \nabla_{\|}^{2} h_{1}=\left(\partial_{t}+\mathbf{v}_{0} \cdot \nabla\right)^{2} \psi_{1}
$$

Vamos supor que as escalas de comprimento em que a tensão superficial torna-se relevante é muito menor que as escalas de variação espacial do fluxo de fundo. Com esta aproximação, temos

$$
\mathrm{g}\left(\partial_{t}+\mathbf{v}_{0} \cdot \nabla\right) h_{1}-\frac{\alpha}{\rho} \nabla_{\|}^{2}\left(\partial_{t}+\mathbf{v}_{0} \cdot \nabla\right) h_{1}=\left(\partial_{t}+\mathbf{v}_{0} \cdot \nabla\right)^{2} \psi_{1}
$$

Utilizando (6.8) na anterior, vem a seguinte equação para as perturbações sobre o potencial de velocidades em torno da superfície do fluido:

$$
\left(\partial_{t}+\mathbf{v}_{0}^{\|} \cdot \nabla_{\|}\right)^{2} \psi_{1}^{(0)}-h_{0} \mathrm{~g} \nabla_{\|}^{2} \psi_{1}^{(0)}=-\frac{\alpha h_{0}}{\rho} \nabla_{\|}^{4} \psi_{1}^{(0)} .
$$

No referencial em que o fluido está em repouso, temos a seguinte relação de dispersão para as ondas na superfície do fluido:

$$
\omega^{2}=g h_{0} \mathrm{k}^{2}\left[1-\left(\frac{\mathrm{k}}{\rho \mathrm{g} / \alpha}\right)^{2}\right] .
$$

Logo, os modos com comprimentos de onda pequenos em relação a $\alpha / \rho$ g são sensíveis à tensão superficial. O modelo de ondas em superfícies, então, também pode prestar-se à investigação experimental de efeitos da mudança da relação de dispersão para comprimentos de onda curtos em teoria de campos clássicos em espaços curvos.

\subsection{Cordas tensionadas}

Suponha uma corda de densidade de massa $\mu=\mu(s)$ e tensão $\tau=\tau(s)$, ambas por unidade de comprimento. Aqui $s$ é o comprimento da corda partindo de algum ponto de referência. Vamos considerar que as vibrações sejam suficientemente pequenas, em relação ao comprimento $L$ da corda quando em equilíbrio, para que $\mu$ e $\tau$ não sejam sensíveis a mudanças do perfil da corda. A ação $S$ para estas vibrações pode ser escrita como

$$
S=-\frac{1}{2} \int_{t_{1}}^{t_{2}} d t \int_{0}^{L} d s\left\{\mu(s)\left[\partial_{t} \phi(s, t)\right]^{2}-\tau(s)\left[\partial_{s} \phi(s, t)\right]^{2}\right\} .
$$

A velocidade $c$ das vibrações na corda é dada por

$$
c^{2}(s)=\frac{\tau(s)}{\mu(s)}
$$


e eliminando $\tau(s)$, temos que a ação do campo de vibrações torna-se

$$
S=-\frac{1}{2} \int_{t_{1}}^{t_{2}} d t \int_{0}^{L} d s \mu(s)\left\{\left[\partial_{t} \phi(s, t)\right]^{2}-c^{2}(s)\left[\partial_{s} \phi(s, t)\right]^{2}\right\} .
$$

Vamos definir a variável $\chi$ através da seguinte relação:

$$
d \chi=c^{-1}(s) d s
$$

Substituindo em (6.10), vem que

$$
S=-\frac{1}{2} \int_{t_{1}}^{t_{2}} d t \int_{\chi(0)}^{\chi(L)} d \chi a^{2}(\chi)\left\{\left[\partial_{t} \tilde{\phi}(\chi, t)\right]^{2}-\left[\partial_{\chi} \tilde{\phi}(\chi, t)\right]^{2}\right\},
$$

onde a função $a^{2}[\chi(s)]$ é dada por

$$
a^{2}[\chi(s)]=c(s) \mu(s)
$$

Esta última é a ação de um campo escalar sem massa definido em um espaço-tempo bidimensional. As coordenadas que escolhemos, $t$ e $\chi$, são tais que a métrica é conforme à métrica do espaço-tempo plano.

A coordenada $\chi$ é

$$
\chi=\int \frac{d s}{c(s)},
$$

e a constante de integração é ajustada para definirmos a origem. Vemos que $\chi$ dá a medida do tempo que um pulso que se propaga na corda leva para percorrer uma distância $\Delta s$.

O horizonte de eventos acústico é uma hiperfície a partir da qual um sinal sonoro tem seu comprimento de onda infinitamente alongado. Então, para construirmos um horizonte de eventos em uma corda basta ajustarmos a forma de $c^{2}$ como função de $s$ de forma tal que

$$
\chi=\int_{s_{\mathcal{H}}}^{s} \frac{d s}{c(s)},
$$

seja divergente. Sendo assim, duas frentes de ondas serão separas por um intervalo de tempo infinito, ou seja, o sinal tem um comprimento de onda enorme. Vamos definir o horizonte de eventos $\mathcal{H}$ como a hiperfície tal que a coordenada espacial $s_{\mathcal{H}}$ satisfaz

$$
c\left(s_{\mathcal{H}}\right)=0 .
$$

É, então, necessário que no ponto $s_{\mathcal{H}}$ da corda a tensão seja nula. 
Considere um espaço-tempo estático e esfericamente simétrico. Neste caso, em coordenadas tais que a secção espacial é isotrópica, o elemento de linha tem a forma

$$
d s^{2}=-A^{2}(\bar{r}) d t^{2}+B^{2}(\bar{r}) d \mathbf{x}^{2} .
$$

Então, a ação de um campo escalar sem massa será

$$
S=-\frac{1}{2} \int d^{4} x A(\bar{r}) B^{3}(\bar{r})\left[\frac{1}{A^{2}(\bar{r})}\left(\partial_{t} \phi\right)^{2}-\frac{1}{B^{2}(\bar{r})}(\nabla \phi)^{2}\right]
$$

Para ondas esfericamente simétricas, $\phi=\phi(\bar{r}, t)$, temos

$$
S=-\frac{1}{2} \int_{t_{1}}^{t_{2}} d t \int \bar{r}^{2} d \bar{r} d \Omega A(\bar{r}) B^{3}(\bar{r})\left[\frac{1}{A^{2}(\bar{r})}\left(\partial_{t} \phi\right)^{2}-\frac{1}{B^{2}(\bar{r})}\left(\partial_{\bar{r}} \phi\right)^{2}\right] .
$$

Comparando (6.9) com (6.11), as dinâmicas são idênticas se fizermos as seguintes correspondências:

$$
s=\bar{r}, \quad \mu=\bar{r}^{2} \frac{B^{3}}{A}, \quad \tau=\bar{r}^{2} A B, \quad c^{2}=\frac{A^{2}}{B^{2}} .
$$

Neste espaço-tempo teremos um horizonte de eventos em $\bar{r}=\bar{r}_{\mathcal{H}}$ quando $A^{2}\left(\bar{r}_{\mathcal{H}}\right)=0$. Isto implica que $\tau$ e $c^{2}$ são nulos sobre o horizonte.

\subsection{Modelo análogo via análise lagrangeana}

Suponha que temos um campo escalar $\phi$ cuja densidade de lagrangeana $\mathcal{L}$ é tal que $\mathcal{L}=\mathcal{L}\left(\phi, \partial_{\mu} \phi\right)$. Seja $\phi_{0}$ a solução da equação de Euler-Lagrange,

$$
\partial_{\alpha}\left[\frac{\partial \mathcal{L}}{\partial\left(\partial_{\alpha} \phi\right)}\left(\phi_{0}, \partial_{\mu} \phi_{0}\right)\right]-\frac{\partial \mathcal{L}}{\partial \phi}\left(\phi_{0}, \partial_{\mu} \phi_{0}\right)=0
$$

Considere pequenas perturbações sobre esta solução de fundo.

$$
\phi=\phi_{0}+\epsilon \phi_{1}+\frac{\epsilon^{2}}{2} \phi_{2}+O\left(\epsilon^{3}\right) .
$$

Expandindo a densidade de lagrangeana $\mathcal{L}$ em torno de $\phi_{0}$, temos

$$
\begin{gathered}
\mathcal{L}\left(\phi, \partial_{\mu} \phi\right)=\mathcal{L}\left(\phi_{0}, \partial_{\mu} \phi_{0}\right)+\epsilon\left[\phi_{1} \frac{\partial \mathcal{L}}{\partial\left(\partial_{\alpha} \phi\right)}+\partial_{\alpha} \phi_{1} \frac{\partial \mathcal{L}}{\partial\left(\partial_{\alpha} \phi\right)}\right]+\frac{\epsilon^{2}}{2}\left[\phi_{2} \frac{\partial \mathcal{L}}{\partial\left(\partial_{\alpha} \phi\right)}+\partial_{\alpha} \phi_{2} \frac{\partial \mathcal{L}}{\partial\left(\partial_{\alpha} \phi\right)}\right]+ \\
+\frac{\epsilon^{2}}{2}\left[\phi_{1}^{2} \frac{\partial^{2} \mathcal{L}}{\partial \phi^{2}}+2 \phi_{1} \partial_{\alpha} \phi_{1} \frac{\partial^{2} \mathcal{L}}{\partial\left(\partial_{\alpha} \phi\right) \partial \phi}+\partial_{\alpha} \phi_{1} \partial_{\beta} \phi_{1} \frac{\partial^{2} \mathcal{L}}{\partial\left(\partial_{\alpha} \phi\right) \partial\left(\partial_{\beta} \phi\right)}\right]+O\left(\epsilon^{3}\right) .
\end{gathered}
$$


Já que $\phi_{0}$ satisfaz a equação de Euler-Lagrange, podemos utilizá-la na segunda e terceira parcelas de (6.12) para transformá-las em termos de borda. Como estamos interessados em variações da ação de forma que as condições inicial e de contorno sejam fixas, podemos desprezar tais termos. Por fim, obtemos a seguinte forma para a ação:

$S[\phi]=S\left[\phi_{0}\right]+\frac{\epsilon^{2}}{2} \int d^{4} x\left[\phi_{1}^{2} \frac{\partial^{2} \mathcal{L}}{\partial \phi^{2}}+2 \phi_{1} \partial_{\alpha} \phi_{1} \frac{\partial^{2} \mathcal{L}}{\partial\left(\partial_{\alpha} \phi\right) \partial \phi}+\partial_{\alpha} \phi_{1} \partial_{\beta} \phi_{1} \frac{\partial^{2} \mathcal{L}}{\partial\left(\partial_{\alpha} \phi\right) \partial\left(\partial_{\beta} \phi\right)}\right]+O\left(\epsilon^{3}\right)$.

Variando a ação acima e impondo que ela deva anular-se ordem a ordem, vem que o primeiro termo é identicamente nulo, já que estamos supondo que $\phi_{0}$ é solução da equação de Euler-Lagrange, e o segundo é nulo se

$$
\partial_{\alpha}\left[\frac{\partial^{2} \mathcal{L}}{\partial\left(\partial_{\alpha} \phi\right) \partial\left(\partial_{\beta} \phi\right)} \partial_{\beta} \phi_{1}\right]+\left[\frac{\partial^{2} \mathcal{L}}{\partial\left(\partial_{\alpha} \phi\right) \partial \phi}-\frac{\partial^{2} \mathcal{L}}{\partial \phi^{2}}\right] \phi_{1}=0 .
$$

A partir da equação acima podemos fazer as seguintes identificações. O termo entre colchetes na primeira parcela pode ser escrito como

$$
\sqrt{|g|} g^{\alpha \beta}=\frac{\partial^{2} \mathcal{L}}{\partial\left(\partial_{\alpha} \phi\right) \partial\left(\partial_{\beta} \phi\right)}
$$

Assim, o módulo do determinante do tensor métrico será

$$
|g|= \pm \operatorname{det}\left\{\frac{\partial^{2} \mathcal{L}}{\partial\left(\partial_{\alpha} \phi\right) \partial\left(\partial_{\beta} \phi\right)}\right\}
$$

O sinal \pm depende da signatura da métrica que, em última análise, depende da estrutura de $\mathcal{L}$. A segunda parcela será posta na seguinte forma:

$$
V[\phi]=\left[ \pm \operatorname{det}\left\{\frac{\partial^{2} \mathcal{L}}{\partial\left(\partial_{\alpha} \phi\right) \partial\left(\partial_{\beta} \phi\right)}\right\}\right]^{\frac{1}{2}}\left[\frac{\partial^{2} \mathcal{L}}{\partial\left(\partial_{\alpha} \phi\right) \partial \phi}-\frac{\partial^{2} \mathcal{L}}{\partial \phi^{2}}\right] .
$$

Em termos das novas quantidades, temos a seguinte equação para as perturbações $\phi_{1}$ :

$$
\frac{1}{\sqrt{|g|}} \partial_{\alpha}\left(\sqrt{|g|} g^{\alpha \beta} \partial_{\beta} \phi_{1}\right)+\mathrm{V}\left[\phi_{0}\right] \phi_{1}=0
$$

Logo, as perturbações, em primeira ordem, obedecem a equação de um campo escalar definido sobre um espaço-tempo curvo cuja métrica é a matriz inversa de $g^{\alpha \beta}$. O termo extra que aparece na equação pode ser interpretado como um termo tipo potencial; em geral, pode conter combinações de $\phi_{0}$ e suas derivadas. No caso em que a estrutura de $\mathcal{L}$ é tal que a métrica é lorentziana, temos que a equação (6.13) é do tipo hiperbólica. Assim, admite soluções do tipo onda. 
O resultado (6.13) vale para qualquer densidade de lagrangeana que dependa de um único campo de fundo e suas derivadas primeira no tempo e no espaço. Para lagrangeanas com esta estrutura, uma geometria efetiva para as perturbações em primeira ordem é conseqüência imediata do procedimento de linearização. Entretanto, $\mathcal{L}$ ainda pode depender de derivadas do campo de ordem superior a um. Isto implica em termos além do operador de D'Alembert. Lagrangeanas com este tipo de estrutura e que dêem origem a uma métrica efetiva que seja lorentziana implicam que os modos do campo obedecem uma relação de dispersão que não é linear. 


\section{Capítulo 7}

\section{Conclusão}

Grandes e numerosos são os desafios que precisam ser transpostos para a construção de uma teoria que descreva as características quânticas da interação gravitacional. Com seu resultado, Hawking talvez tenha começado a pavimentar o longo e tortuoso caminho que nos levará a uma teoria quântica da gravitação e, ainda mais incerto, ao vislumbre de leis da Natureza ainda mais profundas. A lacuna de dados experimentais em contextos astrofísicos e cosmológicos, entretanto, faz com que trilhemos este caminho ainda às escuras.

Analogias constituem um artifício lógico de grande poder e beleza. No caso de analogias entre sistemas gravitacionais e de matéria condensada, tal artifício tem se mostrado útil ao lançar luz sobre pontos dúbios dos cálculos de TQCEC e promissor para reproduzir efeitos de TQCEC em laboratório num futuro próximo. É claro que a existência ou não de radiação térmica de fônons em buracos mudos, por exemplo, não corrobora ou invalida a dedução de Hawking para buracos negros. Ainda assim, testes experimentais de efeitos de TQCEC em análogos seriam importantes para colocar sobre bases experimentais sólidas as tecnicalidades matemáticas utilizadas para deduzi-los. Para que isto seja possível, todavia, é ainda necessário enfrentar algumas dificuldades que existem na implementação destas analogias em laboratório.

Ao logo do texto mostramos que as equações da hidrodinâmica para um fluido ideal e barotrópico cujo fluxo é irrotacional e não-relativístico, quando linearizadas sobre um fluxo de fundo inomogêneo, implica, para as perturbações sobre o potencial de velocidades 
de fundo, em uma equação de Klein-Gordon não-maciça definida sobre um espaço-tempo curvo efetivo. A estas perturbações damos o nome de som e ao espaço-tempo efetivo o nome de espaço-tempo acústico. O espaço-tempo acústico é somente percebido pelo som, enquanto que a estrutura causal do laboratório é determinada, em última análise, pela métrica de Minkowski. A analogia entre o escoamento de fluidos e a RG é posta quando supomos uma família de observadores hipotéticos para os quais a estrutura causal é dada pela métrica acústica.

Uma vez de posse da métrica acústica, foi possível mostrar que a analogia é bem posta, já que a métrica acústica é lorentziana e que os espaços-tempo acústicos satisfazem a condição de estabilidade causal. Também foi possível definir os conceitos, em analogia aos de RG, de espaços-tempos acústico estáticos e estacionários, ergoregião acústica, horizonte de eventos sonoro e de gravidade superficial.

Mostramos, então, como construir espaços-tempos acústicos que contenham um horizonte de eventos através de fluxos em uma, duas e três dimensões. Pela simplicidade, e por ser um modelo largamente usado na literatura para tratar problemas relacionados aos modos trans-planckianos, utilizamos uma montagem tipo bocal de Laval para derivar a radiação Hawking. Em particular, utilizamos uma derivação do resultado de Hawking via o espaço de momenta, um caminho útil para tocar na questão dos modos trans-planckianos. No que diz respeito a esta questão, expusemos a forma com que este problema tem sido discutido nos últimos anos: via a mudança da relação de dispersão no setor de comprimentos de onda curtos.

Dissertamos também sobre os modelos baseados em condensados de Bose-Einstein. É esta a classe de modelos mais promissora para se implementar análogos em laboratório, haja visto o nível de controle de gases ultrafrios que os experimentais atingiram na última década. Além disto, mostramos que se considerado o hamiltoniano de muitos corpos que descreve o sistema, a relação de dispersão no setor de comprimentos de onda curtos deixa de ser linear e passa a ser a de Bogoliubov. Logo, os condensados são uma classe de sistemas interessantes para estudarmos os problema dos modos trans-planckianos. De fato, não temos conhecimento de nenhum experimento em andamento no sentido de implementar análogos em condensados ou em qualquer outro sistema. Talvez os experimentais de 
baixas temperaturas tenham muitas outras coisas para se preocuparem no momento.

Até o presente, todos os modelos análogos que existem na literatura, sejam eles baseados em sistemas de matéria condensada ou não, não são capazes de reproduzir a dinâmica ditada pelas equações de campo de Einstein, isto é, somos somente capazes de simular os aspectos cinemáticos da RG1 1 Mesmo assim, são capazes, ao menos em princípio, de reproduzirem o efeito Hawking. Isto mostra que a radiação térmica emitida por buracos negros independe da dinâmica do campo gravitacional. Para que ela seja produzida é necessário e suficiente que a métrica do espaço-tempo de fundo seja lorentziana e que nele exista um horizonte de eventos [59]. Por outro lado, as leis da mecânica de buracos negros e as leis semi-clássicas da termodinâmica de buracos negros são obtidas assumindo-se que são as equação de Einstein que ditam a dinâmica do sistema. Logo, o efeito Hawking pode ocorrer mesmo em situações em que a entropia de Bekenstein é desprovida de sentido [25].

É possível que a contribuição à física da gravitação por parte dos modelos análogos vá além das questões de TQCEC. Talvez eles também sejam capazes de contribuir com a questão da massa gravitacional ativa em RG. Em princípio, deveríamos atribuir três conceitos de massa distintos a um corpo. Um é o conceito de massa inercial. A massa inercial mede o quão difícil é mudar o estado de movimento de um corpo, ou na linguagem da RG, o quão difícil é tirar este corpo de sua geodésica. Esta é a massa que, por exemplo, comparece na segunda lei de Newton. O outro é o conceito de massa gravitacional, que ainda deve ser separado nos conceitos de massa gravitacional ativa e massa gravitacional passiva. A massa gravitacional ativa é aquela que é fonte do campo gravitacional gerado por um corpo e a passiva a que dá o quão forte é o acoplamento entre uma partícula teste e um campo gravitacional externo. Acontece que o princípio da equivalência unifica dois destes conceitos: a massa inercial e a massa gravitacional passiva. A RG, entretanto, não nos dá uma prescrição de como medir o conteúdo de massa gravitacional ativa de um corpo e ainda é motivo de discussão como fazê-lo. Foi mostrado em [60] que perturbações

\footnotetext{
${ }^{1}$ De fato, ainda não há na literatura nenhum resultado indicando que a dinâmica que rege o sistema de matéria condensada que serve para criar a métrica de fundo pode ser colocada na forma das equações de Einstein. Entretanto, em [58 é mostrado que a ação do campo de fundo que descreve o sistema, quando levado em conta o back-reaction devido às flutuações quânticas, tem uma parcela que é proporcional à ação de Einstein-Hilbert.
} 
sobre o potencial de velocidades de um fluido ideal, em certas circunstâncias, são capazes de simular a dinâmica de partículas clássicas maciças. O resultado sugere que a inércia das partículas é um fenômeno que emerge da existência do fluxo de fundo do fluido. Talvez algo semelhante aconteça com a massa gravitacional ativa e seja possível dizer algo sobre esta quantidade em buracos mudos. Até o momento não temos notícia de nenhum trabalho neste sentido.

Apesar de ser uma rota mais viável para testar TQCEC em laboratório, os análogos apresentam suas próprias complicações experimentais. As particularmente desagradáveis são aquelas que tornam difícil a implementação de fluxos supersônicos e, conseqüentemente, a construção de horizontes sônicos. No sentido de contornar estas dificuldades, Barceló et. al. 61], 62 mostraram que não é necessário a formação de um horizonte de eventos acústico num tempo do laboratório finito para produzir o fluxo térmico de partículas. De fato, basta que assintoticamente no futuro o regime supersônico seja atingido exponencialmente. É claro que ainda há a complicação de construir um sistema que permita este tipo de evolução do fluxo. Este resultado guarda alguma semelhança com o problema da radiação emitida por espelhos acelerados [40]. Lá, quando o espelho tende exponencialmente para uma trajetória tipo luz, o espectro da radiação também é térmico. 


\section{Referências Bibliográficas}

[1] BJORKEN, J. D.; DRELL, S. D. Relativistic Quantum Mechanics. New York: McGraw-Hill, 1980.

[2] PARKER, L. Particle creation in expanding universes. Phys. Rev. Lett., v. 21, n. 8, p. $562-564,1968$.

[3] ZEL 'DOVICH, Ya. B. Generation of waves by a rotating body. JEPT Lett., v. 14, n. 4, p. 180 - 182, 1971.

[4] STAROBINSKII, A. A. Amplification of waves reflected from a rotating black hole. JEPT, v. 64, n. 1, p. 48 - 57, 1973.

[5] UnRuH, W. G. Second quatization in Kerr metric. Phys. Rev. D, v. 10, n. 10, p. $3194-3205,1974$.

[6] BEKEnStein, J. D. Black holes and entropy. Phys. Rev. D, v. 7, n. 8, p. 2333 2346, 1973.

[7] BARDEEN, J. M.; CARTER, B.; HAWKING, S. W. 4 laws of black hole mechanics. Comm. Math. Phys., v. 31, n. 2, p. 161 - 170, 1973.

[8] HAWKING, S. W. Black hole explosions? Nature, v. 248, n. 5443, p. 30 - 31, 1974.

[9] HAWKInG, S. W. Particle creation by black holes. Comm. Math. Phys., v. 43, n. 3, p. $199-220,1975$.

[10] WALD, R. M. The history and present status of Quantum Field Theory in Curved Spacetime. [arxiv:gr-qc/0608018 v1]. Disponível em <http://arxiv.org/>. Acessado em março de 2007. 
[11] BARCELÓ, C.; LIBERATI, S.; VISSER, M. Analogue gravity. [arxiv:gr-qc/0505065 v2]. Disponível em <http://arxiv.org/>. Acessado em março de 2006.

[12] VISSER, M.; BARCELÓ, C.; LIBERATI, S. Analogue models of and for gravity. Gen. Reltiv. Gravit., v. 34, n. 10, p. 1719 - 1734, 2002.

[13] ZOLNERKEVIC, I. Acústica de fluidos ideais análoga à Gravitação. 2004. 65 f. Dissertação (Mestrado em Física) - Instituto de Física Teórica, Universidade Estadual Paulista "Júlio de Mesquita Filho", São Paulo, 2004.

[14] UNRUH, W. G. Experimental black hole evaporation? Phys. Rev. Lett., v. 4, n. 21, p. $1351-1353,1981$.

[15] VISSER, M. Acoustic propagation in fluids: an unexpected example of Lorentzian geometry. [arXiv:gr-qc/9311028 v1]. Disponível em <http://arxiv.org/>. Acessado em março de 2006.

[16] UNRUH, W. G. Sonic analogue of black holes and the effects of high frequences on black hole evaporation. Phys. Rev. D, v. 51, n. 6, p. 2827 - 2837, 1995.

[17] JACOBSON, T. Black-hole evaporation and ultrashort distances. Phys. Rev. D, v. 44, n. 6, p. 1731 - 1739, 1991.

[18] JACOBSON, T. On the outgoing black hole modes. Phys. Rev. D, v. 53, n. 12, p. $7082-7088,1996$.

[19] CORLEY, S.; JACOBSON, T. Hawking spectrum and high frequency dispersion. Phys. Rev. D, v. 54, n. 2, p. 1568 - 1585, 1996.

[20] BROUT, R. et al. Hawkig radiation without trans-Planckian frequencies. Phys. Rev. $D$, v. 52, n. 8 , p. $4559-4568,1995$.

[21] CORLEY, S. Particle creation via high frequency dispersion. Phys. Rev. D, v. 55, n. 10, p. $6155-6161,1997$.

[22] VISSER, M. Acoustic black holes: horizons, ergospheres and Hawking radiation. Class. Quantum Grav., v. 15, n. 6, p. 1767 - 1791, 1998. 
[23] BARCELÓ, C. et al. Causal structure of analogue spacetimes. New J. Phys., v. 6, artigo n. 186, 2004. Disponível em <http://www.njp.org>. Acessado em março de 2007.

[24] VOLOVIK, G. The Universe in a Helium droplet. Oxford: Oxford University Press, 2003.

[25] VISSER, M. Hawking radiation without black hole entropy. Phys. Rev. Lett., v. 80, n. 16, p. 3436 - 3439, 1998.

[26] GARAY, L. J. et al. Sonic analog of gravitational black holes in Bose-Einstein condensates. Phys. Rev. Lett., v. 85, n. 22, p. 4643 - 4647, 2000.

[27] BARCELÓ, C.; LIBERATI, S.; VISSER, M. Analogue gravity from Bose-Einstein condensates. Class. Quantum Grav., v. 18, n. 6, p. 1137 - 1156, 2001.

[28] BARCELÓ, C.; LIBERATI, S.; VISSER, M. Analogue models for FRW cosmologies. Int. J. Mod. Phys. D, v. 12, n. 9, p. 1641 - 1649, 2003.

[29] WEINFURTNER, S. Analogue model for an expanding universe. Gen. Relativ. Gravit., v. 37, n. 9, p. 1549 - 1554, 2005.

[30] VISSER, M.; WEINFURTNER, S. Massive Klein-Gordon equation from a BoseEinstein-condensation-based analogue spacetime. Phys. Rev. D, v. 72, n. 4, artigo n. 044020, 2005.

[31] SCHÜTZHOLD, R.; UNRUH, W. G. Hawking radiation in a eletromagnetic waveguide? Phys. Rev. Lett., v. 95, n. 3, artigo n. 031301, 2005.

[32] UNRUH, W. G.; SCHÜTZHOLD, R. On slow light as a black hole analogue. Phys. Rev. D, v. 68, n. 2, artigo n. 024008, 2003.

[33] BALBINOT, R. et al. Backreaction in acoustic black holes. Phys. Rev. Lett., v. 94, n. 16, artigo n. 161302, 2005.

[34] BALBINOT, R. et al. Hawking radiation from acoustic black holes, short distance and backreaction effects. Riv. Nuovo Cim., v. 28, n. 3, p. 1 - 55, 2005. 
[35] FAGNOCCHI, S. Back-reaction in acoustic black holes. J. Phys.: Conf. Ser., v. 33, p. $445-450,2006$.

[36] WALD, R. R. General Relativity. Chicago: The University of Chicago Press, 1984.

[37] FETTER, A. L.; WALECKA, J. D. Theoretical mechanics of particle and continua. New York: McGraw-Hill, 1980.

[38] LANDAU, L. D.; LIFSHITZ, E. M. Fluid Mechanics. New York: Pergamon Press, 1987.

[39] HARRIS, E. G. A pedestrian approach to Quantum Field Theory. New York: WileyInterscience, 1972.

[40] BIRREL, N. D.; DAVIES, P. C. W. Quantum Field Theory in Curved Space. Cambridge: Cambridge University Press, 1982.

[41] WALD, R. R. Quantum Field Theory in Curved Spacetime and Black Hole Thermodynamics. Chicago: The University of Chicago Press, 1994.

[42] MATSAS, G. E. A. Gravitação semi-clássica. Rev. Bras. Ens. Fís., v. 27, n. 1, p. 137 $-145,2005$.

[43] UNRUH, W. G. Notes on black-hole evaporation. Phys. Rev. D, v. 14, n. 4, p. 870 892, 1976.

[44] CRISPINO, L. C. B.; HIGUCHI, A.; MATSAS, G. E. A. The Unruh effect and its applications. [arXiv:gr-qc/0710.5373 v1]. Disponível em <http://arxiv.org/>. Acessado em novembro de 2007.

[45] ABRAMOWITZ, M. E.; STEGUN, I. A. Handbook of Mathematical Functions. New York: Dover, 1965.

[46] BILIC, N. Relativistic acoustic geometry. [arxiv:gr-qc/9908002 v1]. Disponível em $<$ http://arxiv.org/>. Acessado em março de 2007.

[47] BERGLIAFFA, S. E. P. et al. Wave equation for sound in fluids with vorticity. Physica D, v. 191, n. 1 - 2, p. 121 - 136, 2005. 
[48] GARAY, L. J. et al. Sonic black holes in dilute Bose-Einstein condensates. Phys. Rev. A, v. 6, n. 2, artigo n. 023611, 2001.

[49] FEDICHEV, P. O.; FISCHER, U. R. Gibbons-Hawking effect in the sonic de Sitter space-time of an expanding Bose-Einstein-condensed gas. Phys. Rev. Lett., v. 91, n. 24, artigo n. 24047, 2003.

[50] SCHÜTZHOLD, R. Detection Scheme for acoustic quantum radiation in BoseEinstein condensate. Phys. Rev. Lett., v. 91, n. 19, artigo n. 190405, 2006.

[51] CASTIN, Y.; DUM, R. Bose-Einstein condensates in time dependent traps. Phys. Rev. Lett., v. 77, n. 27, p. 5315 - 5319, 1996.

[52] FETTER, A. L.; WALECKA, J. D. Quantum theory of many-particle systems. San Francisco: McGraw-Hill, 1971.

[53] DALFOVO, F. Theory of Bose-Einstein condensation in trapped gases. Rev. Mod. Phys., v. 71, n. 3, p. 463 - 512, 1999.

[54] KOPNIN, N. B. Introduction to Ginzburg-Landau and Gross-Pitaevskii theories for superconductors and superfluids. J. Low Temp. Phys., v. 129, n. 5 - 6, p. 219 - 262, 2002.

[55] JAIN, P. et al. Analog model of a Friedmann-Robertson-Walker universe in BoseEinstein condensates: Application of the classical field method. Phys. Rev. A, v. 76, n. 3, artigo n. 033616, 2007.

[56] SCHÜTZHOLD, R.; UNRUH, W. G. Gravity waves analogues of black holes. Phys. Rev. D, v. 66, n. 4, artigo n. 044019, 2002.

[57] HEYL, J. S. See a black hole on a shoestring. Phys. Rev. D, v. 74, n. 4, artigo n. 064029, 2006.

[58] BARCELÓ, C.; LIBERATI, S.; VISSER, M. Analogue gravity from field theory normal modes? Class. Quantum Grav., v. 18, n. 17, p. 3595 - 3610, 2001. 
[59] VISSER, M. Essential and inessential features of Hawking radiation. Int. J. Mod. Phys. D, v. 12, n. 4, p. 649 - 661, 2003.

[60] MILGROM, M. Massive particles in acoustic space-times: Emergent inertia and passive gravity. Phys. Rev. D, v. 78, n. 8, artigo n. 084005, 2006.

[61] BARCELÓ, C. et al. Hawking-like radiation does not require a trapped region. Phys. Rev. Lett., v. 97, n. 17, artigo n. 171301, 2006.

[62] BARCELÓ, C. et al. Quasi-particle creation by analogue black holes. Class. Quantum Grav., v. 23, n. 17, p. 5341 - 5366, 2006. 